\title{
Spatial Coherence Enhancing Reconstructions for High Angular Resolution Diffusion MRI
}

\author{
Dissertation zur Erlangung des \\ mathematisch-naturwissenschaftlichen Doktorgrades \\ "Doctor rerum naturalium" \\ der Georg-August-Universität Göttingen \\ im Promotionsprogramm Mathematik \\ der Georg-August University School of Science (GAUSS)
}

\author{
vorgelegt von \\ Christoph Rügge \\ aus Herdecke
}

Göttingen, 2015 



\section{Contents}

1 Introduction $\quad 5$

2 Physical Background 9

2.1 The Measurement . . . . . . . . . . . . . . . . . . . . . . 9 9

2.2 Modeling Multiple Fibers . . . . . . . . . . . . . . . . 16

3 Elements from Regularization Theory $\quad 25$

3.1 Linear Inverse Problems . . . . . . . . . . . . . . . . . . 25

3.2 Discretization of Linear Inverse Problems . . . . . . . . . . . . . 29

4 A Penalty for ODFs 37

4.1 Choice of the Regularization Functional . . . . . . . . . . . . . . 37

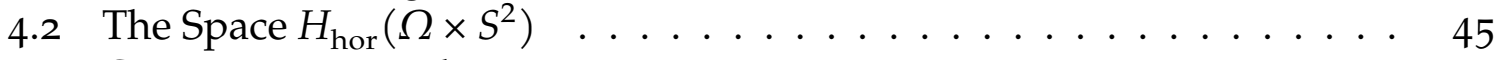

4.3 Convergence Result . . . . . . . . . . . . . . . . 52

5 Discretization and Implementation $\quad 55$

5.1 Discretization of the spherical convolution operator . . . . . . . 55

5.2 Linear basis function on $S^{2} \ldots \ldots \ldots \ldots \ldots$. . . . . . . . . . . . . . . 57

5.3 The Semi-smooth Newton Method . . . . . . . . . . . . . . . . 62

5.4 Spherical Harmonics basis on $S^{2} \ldots \ldots \ldots$. . . . . . . . 68

$\begin{array}{lll}6 & \text { Numerical Experiments } & 73\end{array}$

6.1 Convergence of the projections . . . . . . . . . . . . 73

6.2 Reconstructions of phantom data and comparison of bases . . . . . 75

6.3 Simulated data . . . . . . . . . . . . . . . 80

6.4 Reconstructions of in vivo data and comparison of forward models . . 84

$\begin{array}{lll}7 & \text { Summary } & 97\end{array}$

$\begin{array}{ll}\text { Bibliography } & 99\end{array}$ 



\section{Introduction}

Magnetic Resonance Imaging (MRI) is a non-invasive method to measure characteristics of biological tissue inside human or animal bodies with a wide range of clinical applications as well as applications in biomedical research. There is a large number of techniques tailored to measure and visualize various properties of and processes occurring in biological matter.

Diffusion Weighted MRI (DW-MRI) is a measurement modality that is sensitive to diffusion of water molecules inside the body. It is of particular interest in neuro-scientific research, since it can be used to probe the fibrous structure of white matter in the brain. White matter consists of nerve fibers, called axons, that connect neuronal cell bodies located in the gray matter in the surface region of the brain, and transmit electrical signals between the neurons. While single axons are much too small to be resolved by MRI scans, they are often organized in larger fiber bundles. Inside these bundles, water diffusion is restricted and occurs predominantly along the fibers. Therefore, resolving directional properties of water diffusion can be used to infer the location and orientation of fiber bundles. This information can then be used to analyze the large-scale structure and connectedness of the brain.

DW measurements proceed by measuring a set of full MRI volumes with varying diffusion sensitizing gradients encoding the diffusion in different directions, producing data that is defined on a 6-dimensional space -3 for the spatial location, 3 for diffusion encoding. Due to constraints on measurement time coming from technical, economical and practical considerations, it is not possible to sample this space sufficiently dense. Instead, measured data is acquired only on a lower-dimensional subset of the total space, and additional physical assumptions on the diffusion characteristics are used to make up for the missing data. The most frequently employed measurement modality is called High Angular Resolution Diffusion Imaging (HARDI). Here, data is acquired only on a subset $\Omega \times S^{2}$ with a spatial region $\Omega \subset \mathbb{R}^{3}$ and varying the gradients only in direction, not in magnitude. From this, one tries to reconstruct a suitable measure for the diffusion at each spatial location. The precise quantity to be reconstructed depends on the physical modelling assumptions, but is often a function - a so-called orientation distribution function (ODF) - that assigns to each direction in $S^{2}$ a measure of the relative strength of diffusion into that direction, separately in each voxel.

A number of physical models have been proposed. A notable feature of almost all models is that they describe diffusion independently in each voxel. This is a natural approach due to the separation between the typical scale of diffusion $\left(\sim 10^{-2}-10^{-1} \mathrm{~mm}\right)$ 
and the spatial resolution $(\sim 1-2 \mathrm{~mm})$. Reconstruction then trivially decomposes into a set of rather small ( 100-dimensional) problems in each voxel. This approach, however, disregards the observation that neighboring voxels often have quite similar diffusion characteristics if they belong to the same fibrous structure.

Apart from their immediate utility as local descriptions of diffusion and fiber orientation, ODFs are used as input in further processing steps to reconstruct global characteristics of brain organization. For these, it is important to have accurate, coherent ODFs, in particular for low SNR or coarsely sampled data sets. Therefore, it is interesting to investigate whether the prior knowledge on ODF structure and smoothness can be used to improve the reconstruction.

There are surprisingly few approaches for using spatial regularity in DW-MRI reconstructions. The main challenges one faces when developing such a regularization method are, first, the fact that spatial coherence can not be assumed isotropically, but only along the fiber bundles, while there may be sharp edges perpendicular to them. So it is not clear a priori how to use the prior knowledge without causing artifacts by over-smoothing these edges. Secondly, the resulting problem can become quite large, since it can not be separated into voxels anymore and has to be solved on the total 5-dimensional space, and therefore has to be implemented efficiently. This also precludes the previous point from being approached by too computationally expensive methods.

Spatial regularization was first investigated in [GLTVo9], where similarity is measured by comparing the entire ODFs in nearby voxels using anisotropic weights determined from the data. Smoothing methods that take into account the underlying structure of the domain $\Omega \times S^{2}$ have been suggested for example in [DF11], where linear and non-linear diffusion filters are applied to the reconstructed ODF in a post-processing step, and in [BTV+12], where adaptive smoothing is performed on the HARDI data prior to reconstruction. A completely different approach is taken in [RMA+11], where reconstruction is combined with with fiber tracking in a single step, without the intermediate step of an ODF.

The main contribution of this thesis is the development of a reconstruction algorithm that makes use of spatial coherence to increase noise robustness and to be able to produce accurate, coherent images even with small amounts of data. Based on the linear diffusion filters from [DF11], an anisotropic penalty will be introduced which compares ODFs locally also in the orientational part, and only compares voxels along fibers instead of isotropically. This way, smoothness information can extend for example from single-fiber voxels into adjacent crossings despite the sudden appearance of perpendicular structures which violate the global similarity of the respective ODFs.

Further, theoretical properties of the resulting Tikhonov-type method will be investigated, and convergence will be proved for reconstructions from noisy, discrete data under suitable smoothness assumptions. This will be achieved by studying the properties of a non-standard Sobolev-type space constructed from the anisotropic penalty. 
Finally, the performance of the method for different forward models will be illustrated numerically.

The thesis is organized as follows: In Chapter 2, basic principles of MRI and DWMRI are introduced, and some of the most used physical models are described and discussed.

Chapter 3 gives an overview of basics of regularization theory, with special focus on discretization of constrained, linear inverse problems. Under suitable smoothness assumptions, a convergence result for Tikhonov-type regularization in that setting is proved.

In Chapter 4, the anisotropic regularization penalty is motivated and formally defined. Special consideration is put into examining the properties of a Sobolev-type space defined from this penalty, in particular showing a compact embedding property. This will allow to apply the discrete convergence result form Chapter 3 .

Chapter 5 describes efficient implementation of the method and gives some estimates on the errors of the discrete approximations.

Finally, in Chapter 6, the method is tested on simulated, phantom and in-vivo data. 



\section{Physical Background}

\subsection{The Measurement}

\subsubsection{Basic Principles of MRI}

MRI experiments are based on the behavior of hydrogen nuclei in an external magnetic field. Hydrogen nuclei consist of a single proton. Protons have a quantum mechanical spin, and thereby an intrinsic magnetic moment. The total spin is $1 / 2$, which means that there are two distinct spin states (called up and down). Quantum mechanically, the spin of each proton can be a superposition of these states. If there is no external magnetic field, the energy levels of these states are degenerate, so none of them is preferred over the other. They are therefore occupied with equal probability, and the magnetic moments cancel. When an external magnetic field is applied, the energy levels of the states split, and the lower energy state gets occupied with a higher probability. Averaging over sufficiently many hydrogen spins, this leads to a net magnetic moment.

Macroscopically, the magnetic moment is described by a location- and time-dependent magnetization density $M: \mathbb{R}^{3} \times \mathbb{R} \rightarrow \mathbb{R}^{3}$. If the hydrogen atoms are assumed to be not moving, it can be shown that the time dependence of $M$ in an external magnetic field $B: \mathbb{R}^{3} \times \mathbb{R} \rightarrow \mathbb{R}^{3}$ is described by the ODE

$$
\frac{d}{d t} M(x, t)=-\gamma B(x, t) \times M(x, t),
$$

where $\gamma$ is the gyro-magnetic ratio.

The magnetic field is chosen as a superposition of a strong, constant background field along the $x_{3}$-axis and a weaker time-dependent and spatially varying field:

$$
B(x, t)=B_{3}^{0} e_{3}+\tilde{B}(x, t),
$$

where $\|\tilde{B}(x, t)\| \ll\left|B_{3}^{0}\right|$. For this case, equation (2.1) has been extended by Bloch [Blo46] to include relaxation effects, yielding the Bloch equation

$$
\frac{d}{d t} M(x, t)=-\gamma B(x, t) \times M(x, t)-T^{-1}\left(M(x, t)-M^{\mathrm{eq}}(x)\right),
$$


where $T=\operatorname{diag}\left(T_{2}, T_{2}, T_{1}\right), T_{1}>0$ is the longitudinal relaxation time, $T_{2}<T_{1}$ is the transverse relaxation time and $M^{\mathrm{eq}}(x)=M_{3}^{\mathrm{eq}}(x) e_{3}$ is the equilibrium magnetization density.

MRI experiments go back to Hahn in 1950 [Hah5o]. The basic principle is that, in the beginning of the experiment, the magnetization vector is aligned with the strong magnetic field $B_{3}^{0}$, and is then excited by a radio frequency pulse and rotated into the $x_{1}-x_{2}$-plane, where it precesses and induces a measureable current in nearby receiver coils. Solutions to (2.2) for the various parts of the experiment, corresponding to different choices of $\widetilde{B}$, will be described in the following.

\section{Precession and Decay}

If $\widetilde{B}=0$, the solution to (2.2) can be written as

$$
M(x, t)=R_{\omega t}^{(3)} \exp \left(-t T^{-1}\right)\left(M(x, 0)-M^{\mathrm{eq}}(x)\right)+M^{\mathrm{eq}}(x),
$$

where

$$
R_{\omega t}^{(3)}=\left(\begin{array}{ccc}
\cos (\omega t) & \sin (\omega t) & 0 \\
-\sin (\omega t) & \cos (\omega t) & 0 \\
0 & 0 & 1
\end{array}\right)
$$

is the rotation matrix by angle $\omega t$ around the $x_{3}$-axis. The magnetization precesses around the $x_{3}$-axis with the Larmor frequency $\omega=\gamma B_{3}^{0}$ while decaying exponentially to $M^{\text {eq }}$ on a time scale given by $T_{1}$ and $T_{2}$. In particular, the initial condition for the MRI experiments, i.e. after switching on the background field and waiting for some sufficiently long time, is

$$
M\left(x, t_{0}\right)=M^{\mathrm{eq}}(x)
$$

\section{RF Pulses}

Radio frequency (RF) pulses are used to flip the magnetization vector from its equilibrium position $M^{\mathrm{eq}}$ into the $x_{1}-x_{2}$-plane. They are usually much shorter than the relaxation times, so relaxation effects will be ignored here. The varying magnetic field is chosen as

$$
\tilde{B}(x, t)=B^{\mathrm{RF}} R_{\omega t}^{(3)} e_{1},
$$

with $B^{\mathrm{RF}} \in \mathbb{R}$. Setting $M(x, t)=R_{\omega\left(t-t_{0}\right)}^{(3)} \tilde{M}(x, t)$, equation (2.1) becomes

$$
\frac{d}{d t} \tilde{M}(x, t)=-\gamma B^{\mathrm{RF}} e_{1} \times \tilde{M}(x, t)
$$

so in the rotating coordinate frame, $\tilde{M}$ precesses around the $x_{1}$-axis. The solution to $(2.3)$ is

$$
M(x, t)=R_{\omega\left(t-t_{0}\right)}^{(3)} R_{\nu\left(t-t_{0}\right)}^{(1)} M^{\mathrm{eq}}(x)
$$


with $v=\gamma B^{\mathrm{RF}}$. By choosing the field strength $B^{\mathrm{RF}}$ and the pulse length, different flip angles can be achieved. In the following, the flip angle is taken to be $90^{\circ}$, so that after the RF pulse (which is defined to be $t=0$ ), one has

$$
M(x, 0)=M_{3}^{\mathrm{eq}}(x) e_{1}=\rho(x) e_{1},
$$

where $\rho$ denotes the spin density and the proportionality between $M^{\mathrm{eq}}$ and $\rho$ is due to the fact that all spins are excited by the RF pulse in the same way. The constant of proportionality was set to 1 for simplicity.

The above derivation worked because the frequency of the RF pulse was chosen identical to the Larmor frequency, so the magnetic field is "in phase" with the precessing magnetization vector. If the RF pulses differs too much from the Larmor frequency, different contributions add up destructively and do not lead to a flip of magnetization.

In many MRI experiments, the background field is actually not chosen as a constant, but varies linearly along one of the coordinate axes during the RF pulse. In this case, the RF frequency coincides with the Larmor frequency only in a slice of the volume, and the initial condition becomes:

$$
M(x, 0)=\rho(x)\left(e_{1} \cos \left(\alpha\left(x_{3}-z\right)\right)+e_{3} \sin \left(\alpha\left(x_{3}-z\right)\right)\right),
$$

where $\alpha: \mathbb{R} \rightarrow \mathbb{R}$ is sharply peaked around 0 and the background field was taken to vary along the $x_{3}$ axis (without loss of generality), with the RF pulse being in resonance at $x_{3}=z$. As it will be described below, the non-excited spins, i.e. those that are still directed along $e_{3}$ after the RF pulse, do not contribute to the measured signal, so the measurement is effectively restricted to the slice around $x_{3}=z$. This is called slice selection.

\section{Gradient Field}

Until now, the magnetic fields have been spatially constant. To be able to reconstruct the spin density $\rho$ as a function of space, spatially varying magnetic fields are needed. They are chosen as gradient fields

$$
\widetilde{B}(x, t)=(G(t) \cdot x) e_{3}
$$

where $G: \mathbb{R} \rightarrow \mathbb{R}^{3}$. Defining

$$
M_{\perp}(x, t):=M_{1}(x, t)+i M_{2}(x, t),
$$

equation (2.2) is equivalent to

$$
\begin{gathered}
\frac{d}{d t} M_{\perp}(x, t)=-i \gamma B_{3}(x, t) M_{\perp}(x, t)-T_{2}^{-1} M_{\perp}(x, t) \\
\frac{d}{d t} M_{3}(x, t)=-T_{1}^{-1}\left(M_{3}(x, t)-\rho(x)\right),
\end{gathered}
$$


and the initial conditions are

$$
M_{\perp}(x, 0)=\rho(x) \cos \left(\alpha\left(x_{3}-z\right)\right), \quad M_{3}(x, 0)=\rho(x) \sin \left(\alpha\left(x_{3}-z\right)\right) .
$$

This is solved by

$$
\begin{gathered}
M_{\perp}(x, t) \equiv M_{\perp}(x, z, t)=\exp \left(-\frac{t}{T_{2}}\right) \exp \left(-i \gamma \int_{0}^{t} B_{3}(x, s) d s\right) \rho(x) \cos \left(\alpha\left(x_{3}-z\right)\right) \\
M_{3}(x, t) \equiv M_{3}(x, z, t)=\left(1-\exp \left(-\frac{t}{T_{1}}\right)\right) \rho(x) \sin \left(\alpha\left(x_{3}-z\right)\right)
\end{gathered}
$$

The rotating magnetic moment $M_{\perp}$ induces a current in receiver coils outside the object under examination, which constitutes the measured signal. It is proportional to the sum of all transverse magnetic moments. Define the $k$-vector by

$$
k(t):=\frac{\gamma}{2 \pi} \int_{0}^{t} G(s) d s
$$

Then the measured signal at time $t$ is

$$
\begin{aligned}
\tilde{S}(z, t) & =\int_{\mathbb{R}^{3}} M_{\perp}(x, z, t) d x \\
& =\exp \left(-\frac{t}{T_{2}}\right) \exp (-i \omega t) \int_{\mathbb{R}^{3}} \rho(x) \cos \left(\alpha\left(x_{3}-z\right)\right) \exp (-2 \pi i k(t) \cdot x) d x .
\end{aligned}
$$

The spatially constant high-frequency precession term $\exp (-i \omega t)$ is rather uninteresting and will be ignored from here on, as it can be removed in experiments. If the spatially dependent flip angle $\alpha$ is sufficiently concentrated around 0 , the signal can be approximated by

$$
\left.\tilde{S}(z, t) \approx \exp \left(-\frac{t}{T_{2}}\right) \int_{\mathbb{R}^{2}} \rho(x) \exp (-2 \pi i k(t) \cdot x)\right|_{x_{3}=z} d x_{1} d x_{2}
$$

up to a constant that only rescales the unknown density $\rho$ and is therefore omitted. The interpretation of this is that the signal is essentially given by the Fourier transform of the spin density $\rho(\cdot, \cdot, z)$, evaluated along a continuous trajectory $t \mapsto k(t)$.

The exponential factor in (2.6) leads to a rapid decay of the measured signal. This is due to local field inhomogeneities which lead to loss of coherence of the spins constituting the macroscopic magnetization. Several techniques can be used to refocus the spins (echo) and produce a measurable signal. In the following, we will simply omit the exponential factor.

In practice, finitely many samples of the signal are taken at times $t_{1}, t_{2}, \ldots, t_{n}$ and the trajectory is chosen to cover $k$-space, i.e. the domain of definition of the Fourier transform, as good as possible given the constraints on the length of measurement 
and the strength of the gradients $G=k^{\prime}$. This yields the following expression for the signal:

$$
\hat{S}(z, k)=\left.\int_{\mathbb{R}^{2}} \rho(x) \exp (-2 \pi i k \cdot x)\right|_{x_{3}=z} d x_{1} d x_{2}, \quad k \in \mathcal{K},
$$

where $\mathcal{K}=\left\{k\left(t_{j}\right): j=1,2, \ldots, n\right\}$. Often, one chooses $\mathcal{K} \perp e_{3}$.

\subsubsection{Parallel Imaging}

Using (2.7), reconstruction of the unknown spin density $\rho$ from the measured MRI signal is straightforward if the $k$-space trajectory is chosen such that $\mathcal{K}$ is a regular grid. In that case, the inversion can be done by using a Fast Fourier Transform (FFT). However, data acquisition can take quite long in this case, given the number of samples necessary to achieve the desired resolution, especially for 3-dimensional volumes or diffusion weighted images (see Section 2.1.3). In order to improve acquisition times, parallel imaging can be used. This technique sub-samples $k$-space while measuring the signal with multiple receiver coils simultaneously. These coils usually do not cover the full field of view, but instead have spatial sensitivity profiles $c_{j}: \mathbb{R}^{2} \rightarrow \mathbb{C}\left(j=1, \ldots, n_{c}\right)$. Equation (2.7) is replaced by

$$
\hat{S}_{j}(z, k)=\left.\int_{\mathbb{R}^{2}} \rho(x) c_{j}\left(x_{1}, x_{2}\right) \exp (-2 \pi i k \cdot x)\right|_{x_{3}=z} d x_{1} d x_{2}, \quad k \in \mathcal{K}, \quad j=1, \ldots, n_{c} .
$$

The idea behind parallel imaging is to trade $k$-space information for the redundant spatial information in the receiver coils, thus measuring data in parallel instead of serially.

This approach is complicated by the fact that the coil profiles $c_{j}$ depend on the object under investigation and are not known a priori. To overcome this, one generally exploits the fact that coil profiles are very smooth. Widely used linear inversion methods like SENSE [RR93] determine the coil profiles from reference images or a fully sampled center of $k$-space to obtain low-resolution, smoothed versions of $\rho c_{j}$ and $\rho c_{0}$, where $c_{0}$ is the approximately constant profile of a homogeneous body coil. From this, approximations to the $c_{j}$ can be determined up to a constant factor, and the density $\rho$ is reconstructed by inverting the linear mapping $\rho \mapsto\left(\hat{S}_{j}\right)_{j=1}^{n_{c}}=: \hat{S}$ using the full (sub-sampled) $k$-space data.

A disadvantage of these approaches is that calibration errors in the coil profiles can lead to serious artifacts in the reconstructed spin density, and determining the coil profiles only from a part of the available $k$-space data can therefore lead to sub-optimal results. An approach to parallel imaging addressing this problem has been developed in [UHBFo8]. Here, the non-linear map $(\rho, c) \mapsto \hat{S}$ is inverted by an iterative algorithm that is regularized to enforce the smoothness assumptions on the coil profiles, thereby leveraging the full $k$-space data to reconstruct both $\rho$ and $c:=\left(\hat{c}_{j}\right)_{j=1}^{n_{c}}$. 


\subsubsection{Diffusion Weighted MRI}

If the assumption of non-moving hydrogen atoms is dropped to incorporate effects of water diffusion, the treatment in the previous sections is not valid anymore and must be modified. One possible way to do this is to extend the Bloch equations (2.2) with diffusion terms, yielding the Bloch-Torrey equations [Tor56]. However, this approach incorporates the tensor model (cf. Section 2.2.1) at a very early stage and is therefore unsuitable for models that try to extend the tensor model. This section gives a more general, heuristic derivation of the diffusion weighted MR signal.

Consider an atom drifting along a curve $x: \mathbb{R} \rightarrow \mathbb{R}^{3}$ in a time- and space-dependent magnetic field $B_{3}: \mathbb{R} \times \mathbb{R}^{3} \rightarrow \mathbb{R}$ pointing in $x_{3}$-direction. Then, generalizing (2.4) and neglecting relaxation effects, its transversal magnetization $m_{\perp}$ satisfies

$$
\frac{d}{d t} m_{\perp}(t)=-i \gamma B_{3}(x(t), t) m_{\perp}(t)
$$

i.e.

$$
m_{\perp}(t)=\exp \left(-i \gamma \int_{0}^{t} B_{3}(x(s), s) d s\right) m_{\perp}(0) .
$$

As above, $B_{3}$ is taken to be a superposition of a constant field (which is ignored again) and a gradient. For diffusion weighted (DW) images, the Stejskal-Tanner sequence [ST65] is used. It consists of two strong, opposite diffusion encoding gradient pulses at times $t_{0}$ and $t_{1}>t_{0}$, followed by a sequence to sample $k$-space in the way described above. For general choices of the diffusion gradients, it is difficult if not impossible to derive analytical expressions for the magnetization, except under some restrictive assumptions on the diffusion model. A tractable - albeit rather unrealistic - limiting case is the narrow pulse approximation [Ste65], in which the pulses are taken to be Dirac delta functions, i.e.

$$
G(t)=-\frac{q}{2 \pi \gamma}\left(\delta\left(t-t_{0}\right)-\delta\left(t-t_{1}\right)\right)+\tilde{G}(t),
$$

where $q \in \mathbb{R}^{3}$ and the time-dependent gradient field $\tilde{G}$ is used to sample $k$-space. For $t>t_{1}$, we have

$$
m_{\perp}(t)=\exp \left(-2 \pi i q \cdot\left(x\left(t_{1}\right)-x\left(t_{0}\right)\right)\right) \exp \left(-i \gamma \int_{0}^{t} \tilde{G}(s) \cdot x(s) d s\right) m_{\perp}(0)
$$

Define $k(t):=(2 \pi)^{-1} \gamma \int_{0}^{t} \tilde{G}(s) d s$. For $t>t_{1}$, this coincides with the definition of $k$ in (2.5). The exponential function above depends on the complete path $t \mapsto x(t)$ of the particle. We would like to approximate this by an expression only involving the initial position $x(0)$. The intuition behind this is that the average diffusion length during the measurement time is usually much smaller than the spatial resolution, and therefore the atom should "appear stationary" to the $k$-space gradients. However, an estimate of the modulus of the integral in (2.8) for arbitrary paths $x$ can not be better than proportional to $\int_{0}^{t}\|\tilde{G}(s)\| d s$, which is a detail of the chosen gradient sequence and 
not connected to the spatial resolution. Therefore, some assumptions on the path $x$ are needed.

We suggest to view it as a Wiener process, i.e. a stochastic process modelling Brownian motion. This means that for every $t, x(t)$ is viewed as a random variable in $\mathbb{R}^{3}$ fulfilling certain conditions. Going into detail here would be much too lengthy; an introduction to integration of stochastic processes can be found in [Økso3].

The exponent in (2.8) can be rewritten as ${ }^{1}$

$$
-i \gamma \int_{0}^{t} \tilde{G}(s) \cdot x(s) d s=-2 \pi i k(t) \cdot x(0)+2 \pi i \int_{0}^{t}(k(s)-k(t)) \cdot d x(s) .
$$

The integral itself is a real-valued random variable. It has zero mean value, and its variance can be shown to be

$$
\begin{aligned}
\operatorname{Var}\left(\int_{0}^{t}(k(s)-k(t)) \cdot d x(s)\right) & =D \int_{0}^{t}\|k(s)-k(t)\|^{2} d s \\
& \leq 4 D t \sup _{0 \leq s \leq t}\|k(s)\|^{2},
\end{aligned}
$$

where $D$ is the diffusion constant of the Brownian motion. $\sqrt{2 D t}$ can be interpreted as the average diffusion length during time $t$, while the maximum $k$-value is the inverse of the spatial resolution. Typical diffusion coefficients in white matter are roughly $D \approx 10^{-3} \mathrm{~mm}^{2} \mathrm{~s}^{-1}$, so the square of the diffusion length during measurement time $t \approx 1 \mathrm{~s}$ can be estimated as $2 D t \approx 2 \times 10^{-3} \mathrm{~mm}^{2}$. On the other hand, the typical spatial resolution for diffusion weighted images is about 1 to $2 \mathrm{~mm}$, so the variance in equation (2.9) is approximately $10^{-3}$. Therefore, it appears reasonable to neglect this term in (2.8), i.e.

$$
m_{\perp}(t) \approx \exp \left(-2 \pi i q \cdot\left(x\left(t_{1}\right)-x\left(t_{0}\right)\right)\right) \exp (-2 \pi i \gamma k(t) \cdot x(0)) m_{\perp}(0) .
$$

The measured signal is again the sum of all individual phases. Let $\rho(x)$ be the spin density at $x \in \mathbb{R}^{3}$ and $p(x, r)$ be the probability for a particle that is initially at $x$ to drift to $x+r$ between the diffusion gradient pulses $^{2} . p$ is called the diffusion propagator. The signal is

$$
\hat{S}(z, t, q)=\left.\int_{\mathbb{R}^{3}} \int_{\mathbb{R}^{2}} \rho(x) p(x, r) \exp (-2 \pi i k(t) \cdot x) \exp (-2 \pi i q \cdot r)\right|_{x_{3}=z} d x_{1} d x_{2} d r,
$$

which, by sampling along a trajectory in $k$-space, becomes

$$
\hat{S}(z, k, q)=\left.\int_{\mathbb{R}^{3}} \int_{\mathbb{R}^{2}} \rho(x) p(x, r) \exp (-2 \pi i k \cdot x) \exp (-2 \pi i q \cdot r)\right|_{x_{3}=z} d x_{1} d x_{2} d r
$$

for $k \in \mathcal{K} \subset \mathbb{R}^{3}$. Diffusion weighted measurements can also be combined with parallel imaging in the same way as described in Section 2.1.2.

\footnotetext{
${ }^{1}$ By [Økso3, Theorem 4.1.5 (Integration by parts)], one has to assume that $k$ has bounded variation, which is the same as saying that $\tilde{G}$ is integrable.

${ }^{2}$ Actually, it should be the probability to drift from some point $x\left(t_{0}\right)$ near $x=x(0)$ to $x\left(t_{1}\right)=x\left(t_{0}\right)+r$ between the pulses, but the difference is expected to be small.
} 


\subsubsection{Phase Artifacts}

Due to the strong gradients employed, diffusion weighted images are in general very sensitive to all kinds of particle motion. In particular, unavoidable effects like brain pulsing and patient movement introduce phase artifacts into the measured data. A simple way of formally incorporating these coherent movements into the signal equation is by translating the diffusion propagator, i.e. by replacing $p(x, r)$ in (2.10) with $p(x, r+\tilde{\varphi}(x, q))$, where the translation vector $\tilde{\varphi}$ depends on both the spatial position (since e.g. brain pulsing is spatially inhomogeneous) and the diffusion gradient (since measurements for different $q$ occur at different times). This leads to

$$
\begin{aligned}
\hat{S}(z, k, q)=\int_{\mathbb{R}^{3}} \int_{\mathbb{R}^{2}} \rho(x) \exp (2 \pi i \varphi(x, q)) & p(x, r) \\
& \times\left.\exp (-2 \pi i k \cdot x) \exp (-2 \pi i q \cdot r)\right|_{x_{3}=z} d x_{1} d x_{2} d r,
\end{aligned}
$$

where $\varphi(x, q)=q \cdot \tilde{\varphi}(x, q)$.

Since very little is known about the structure and smoothness of the phase artifacts, the most frequently employed way to handle them is to remove them by splitting the reconstruction into two parts: At first, the spatial Fourier transform is inverted for each diffusion gradient $q$ separately, either by an FFT in the fully sampled case, or by one of the algorithms for parallel imaging outlined above. Inversion of the $q$-space Fourier transform is done in a separate step. Between those steps, the phase maps are removed by taking the modulus, obtaining

$$
S(x, q):=|\rho(x)|\left|\int_{\mathbb{R}^{3}} p(x, r) \exp (-2 \pi i q \cdot r) d r\right|=:|\rho(x) \| \hat{p}(x, q)|
$$

The spin density $\rho$ is real and positive, and the natural symmetry assumption $p(x, r)=$ $p(x,-r)$ on the diffusion propagator leads to its Fourier transform w.r.t. $r$ being real. In the tensor model (see below), it is also positive; for general $p$, this is taken as an additional assumption, so

$$
S(x, q)=\rho(x) \hat{p}(x, q) .
$$

The collection $S(\cdot, q)$ for all measured $q$-values is called magnitude images. Inversion of the $q$-space encoding will be described in Section 2.2.

\subsection{Modeling Multiple Fibers}

The aim of DW-MRI is to reconstruct information about the diffusion propagator $p$ in equations (2.10) and (2.12). For a straight-forward Fourier inversion of (2.10), the signal has to be sampled on a sufficiently fine grid in $\mathbb{R}^{6}$, requiring very long acquisition times and thereby posing practical, economical as well as technical problems. As in the case of parallel imaging, the remedy is to acquire less $q$-space data than needed 
for an inverse Fourier transform by measuring only on a subset $\mathcal{Q} \subset \mathbb{R}^{3}$. Unlike that case, this is however not compensated for by multiple receiver coils or other technical measures. Instead, physical assumptions on the diffusion propagator are used to extend the measured data from the subset $\mathcal{Q}$ to the whole $q$-space. In many cases, the data is measured only on a sphere in $q$-space (single-shell) with the aim of achieving high angular resolution of the diffusion propagator while neglecting information about diffusion length. This mode of measurement is called High Angular Resolution Diffusion Imaging (HARDI).

This section describes some of the most important models. These models generally describe the "microscopic" diffusion process on length scales much smaller than a voxel, and are therefore independent of the voxel variable $x$ of the diffusion propagator $p(x, \cdot)$

\subsubsection{The Tensor Model}

The most widely employed model for DW-MRI is the tensor model (DTI, Diffusion Tensor Imaging), which takes the diffusion propagator to be a Gaussian function, i.e.

$$
p(x, r)=\frac{1}{\sqrt{(2 \pi)^{3} \operatorname{det} D}} \exp \left(-\frac{1}{2 \tau} r^{T} D^{-1}(x) r\right),
$$

where $D(x) \in \mathbb{R}^{3 \times 3}$ is the positive definite diffusion tensor and $\tau=t_{1}-t_{0}$ is the time between the diffusion gradient pulses. The motivation for this choice is that $p(x, \cdot)$ is the solution at time $t=\tau$ to the diffusion equation

$$
\partial_{t} u(r, t)=\nabla_{r} \cdot D(x) \nabla_{r} u(r, t), \quad u(r, 0)=\delta(r) .
$$

Inserting $p$ into (2.12) yields

$$
S(x, q)=\rho(x) \exp \left(-2 \pi^{2} \tau q^{T} D(x) q\right) .
$$

The quantity $b:=2 \pi^{2} \tau\|q\|^{2}$ is called $b$-value and is a measure for the strength of diffusion encoding sequence. In the tensor model, the narrow pulse approximation made in derivation above can actually be dropped, leading to corrections to the definition of the $b$-value for taking into account the finite gradient pulse length. In the following however, these technical issues will be ignored. Note that in order to achieve a high $b$-value, one can either use strong gradients $\|q\|$ or a long separation time $\tau$ between the pulses.

Since $D$ is symmetric, the tensor model has only 7 unknown parameters per voxel, so in principle one unweighted image for the reconstruction of $\rho$ and six diffusion weighted images suffice to determine $D$. In practice, more images are used to increase noise robustness, but measurement time is still only a fraction of what would be needed for a full $q$-space sampling. Another advantage of this model is the clear interpretation of the diffusion tensor, from which a number of important measures can be computed: 
- The apparent diffusion coefficient (ADC) $\langle D\rangle=\operatorname{tr} D / 3$, reflecting the total amount of diffusion,

- the fractional anisotropy

$$
\mathrm{FA}(D)=\left(\frac{3 \operatorname{tr}\left((D-\langle D\rangle \mathbb{1})^{2}\right)}{2 \operatorname{tr}\left(D^{2}\right)}\right)^{\frac{1}{2}},
$$

reflecting the anisotropy of the diffusion by measuring the deviation from a diagonal matrix, and

- the estimated fiber direction, which is taken to be the eigenvector to the largest eigenvalue of $D$.

As the last point shows, the model can only estimate a single fiber direction per voxel, which is a serious drawback since about $30 \%$ of the voxels in white matter are expected to contain more than one fiber. [BBJ+O7]

A considerable number of models and methods has been suggested to overcome these limitations of DTI for HARDI data acquisitions. Some of these will be outlined in the next sections.

\subsubsection{Q-Ball Imaging}

In this section, we omit the spatial variable for notational simplicity. The model described here applies separately in each voxel.

Q-Ball imaging does not try to reconstruct the full diffusion propagator but instead focuses on the direction of diffusion, ignoring length information. This is done by defining the orientation distribution function (ODF)

$$
\psi_{n}(u):=\int_{0}^{\infty} p(t u) t^{n} d t, \quad n \in \mathbb{N} .
$$

The original Q-Ball imaging, developed by Tuch in [Tuco4], used $n=0$. The mathematical basis for $\mathrm{Q}-\mathrm{Ball}$ reconstruction is the following observation:

Lemma 2.1. Let $p \in S\left(\mathbb{R}^{3}\right)$ and $u \in S^{2}$. Then

$$
\int_{-\infty}^{\infty} p(t u) d t=\int_{k \perp u} \hat{p}(k) d k .
$$

Here, $\mathcal{S}\left(\mathbb{R}^{3}\right)$ is the Schwartz space of functions with rapidly decreasing derivatives of arbitrary order. The theorem actually holds for a much larger class of functions. 
Proof. For $k \perp u$, we have

$$
\int_{v \perp u} \int_{-\infty}^{\infty} p(v+t u) d t \exp (-2 \pi i k \cdot v) d v=\hat{p}(k) .
$$

Fourier inversion on the two-dimensional plane perpendicular to $u$ yields

$$
\int_{-\infty}^{\infty} p(v+t u) d t=\int_{k \perp u} \hat{p}(k) \exp (2 \pi i k \cdot v) d k .
$$

Setting $v=0$ shows the statement.

This shows that $\psi_{0}$ can be computed by integrating $\hat{p}=S / \rho$ over planes in $q$-space. Since $S$ is only measured on $S^{2}$, the integral has to be approximated in some way. TucH approximates it by integrating over the measured data on the sphere $S^{2}$ only,

$$
\psi_{0}=\mathcal{R} \hat{p},
$$

where $\mathcal{R}: L^{2}\left(S^{2}\right) \rightarrow L^{2}\left(S^{2}\right)$ is the Funk-Radon transform defined by

$$
\mathcal{R} f(u)=\int_{S^{2} \cap\{u\}^{\perp}} f(v) d v,
$$

Despite being quite successful and easy to implement - the Funk-Radon transform is diagonalized by Spherical Harmonics -, this variant of Q-Ball imaging has some drawbacks:

- The choice $n=0$ has unrealistic scaling behavior: if $p$ is given by the tensor model (2.13), larger diffusion tensors $D$ lead to smaller $\psi_{0}$. This has to be compensated by introducing an additional scaling factor to be able to interpret it as a probability distribution.

- The approximation of the integral above effectively assumes $\hat{p}(q)=\hat{p}_{0}(q) \delta(\|q\|-1)$, which not physically justified, not even as an approximation.

- The Funk-Radon transform is compact. While that makes the reconstruction very robust against noise, it also leads to significant blurring of the ODFs.

Later, AgANJ et al. [ALS+10] proposed to use $\psi_{2}$ instead of $\psi_{0}$, which can be interpreted as a probability distribution on $S^{2}$ since it is automatically normalized (solid-angle $Q$-Ball imaging). Motivated by the tensor model, $\hat{p}$ is extended from the measured data on $S^{2}$ to all of $\mathbb{R}^{3}$ by assuming

$$
\hat{p}(q)=\exp \left(-\|q\|^{2} D\left(\frac{q}{\|q\|}\right)\right)
$$


for $q \neq 0$ and some $D: S^{2} \rightarrow \mathbb{R}$ (mono-exponential decay model). This leads to the explicit reconstruction formula

$$
\psi_{2}=\frac{1}{4 \pi}+\frac{1}{16 \pi^{2}} \Delta_{S^{2}} \mathcal{R} \log \left(-\log \left(\frac{S}{\rho}\right)\right),
$$

where $\Delta_{S^{2}}$ is the Laplace-Beltrami operator on $S^{2}$. Since for all smooth functions $f$ on $S^{2}, \int_{S^{2}} \Delta_{S^{2}} f(u) d u=0$, this function is automatically normalized to 1, i.e. can be interpreted as probability distribution.

However, also assumption (2.15) is not physically realistic in general, since $\hat{p}$ in this model is smooth at $q=0$ if and only if it is a Gaussian. Therefore, $p(r)$ decays only polynomially as $\|r\| \rightarrow \infty$ for general functions $D$.

\subsubsection{Spherical Deconvolution}

The spherical deconvolution model, introduced in [TCGCo4], is a heuristic model for single-shell measurements that does not involve the Fourier relation (2.12). Instead, it tries to reconstruct the fiber density, i.e. the number of fibers per solid angle, by using the following assumptions (see the reference cited above for a discussion):

- All fibers are identical, irrespective of direction and location. If the measured response of a fiber in direction $u \in S^{2}$ is given by $E_{u}: S^{2} \rightarrow \mathbb{R}$ - where the signal is assumed to be measured on a unit sphere in $q$-space without loss of generality - and $R \in \mathrm{SO}(3)$ is a rotation matrix, then $E_{R u}=E_{u} \circ R^{-1}$. This implies that the response is rotationally symmetric around the fiber direction.

- The measured signal of a collection of fibers is the superposition of the individual responses, weighted by the number of spins diffusing along the fiber. This can be justified by arguing that there is no significant exchange of hydrogen atoms between different fiber bundles during measurement time.

- The number of spins diffusing along a fiber at $x$ is proportional to $\rho(x)$.

Let $\xi \in S^{2}$ be an arbitrary, fixed vector and choose, for each direction $u \in S^{2}$, a rotation matrix $R_{u} \in \mathrm{SO}(3)$ such that $R_{u} \xi=u$. Then for all $u$,

$$
E_{u}=E_{R_{u} \xi}=E_{\xi} \circ R_{u}^{-1} .
$$

If $\psi(x, u)$ is the fiber density in at $x \in \mathbb{R}^{3}$ in direction $u$ and $\rho(x)$ is the spin density at $x$, then the total signal can be written as

$$
\begin{aligned}
S(x, q) & =\rho(x) \int_{S^{2}} E_{u}(q) \psi(x, u) d u \\
& =\rho(x) \int_{S^{2}} E_{\xi}\left(R_{u}^{-1} q\right) \psi(x, u) d u
\end{aligned}
$$


Since $E_{\xi}$ is rotationally symmetric around $\xi$, there exists a function $K:[-1,1] \rightarrow \mathbb{R}$, such that

$$
E_{\xi}(q)=K(q \cdot \xi)
$$

Hence,

$$
S(x, q)=\rho(x) \int_{S^{2}} K(q \cdot u) \psi(x, u) d u .
$$

From this, there are two ways of treating the unknown density $\rho$ : if it is available explicitly from an unweighted magnitude image, (2.17) can be rewritten in terms of the quotient $S / \rho$ (scaled deconvolution). Otherwise, the product $\varphi:=\rho \psi$ can be reconstructed in place of $\psi$ to eliminate $\rho$ (unscaled deconvolution). The convolution kernel $K$ can either be estimated from the data, or be parametrized a priori, for example as a Gaussian

$$
K(t)=\exp \left(-b\left(\alpha+\beta t^{2}\right)\right)
$$

with given $b$-value and fixed eigenvalues $\alpha, \beta \in \mathbb{R}^{+}$.

Unfortunately, the Spherical Deconvolution Model also has a few unrealistic properties:

- If the kernel is positive, as in the frequently used parametrization (2.18), increasing the fiber density $\psi$ leads to an increase of the measured signal. This is to be contrasted to the tensor model, where larger diffusion tensors lead to a decreasing signal, and which is well-established experimentally.

- The assumption of identical fibers does not seem justified. This may be partially alleviated if some of the variation between the signals of different fibers is described by different fiber densities $\psi$ instead of different response functions $K$.

\subsubsection{Multi-shell deconvolution}

If data is measured on more than one shell with varying $b$-values, $\mathcal{Q} \subset \cup_{i=1}^{n} \sqrt{b_{i}} S^{2}$, the convolution model can be extended by using varying single-fiber response functions $\left\{K_{i}\right\}_{i=1}^{n}$

$$
S(x, q)=\rho(x) \int_{S^{2}} K_{i}(q \cdot u) \psi(x, u) d u, \quad\|q\|^{2}=b_{i} .
$$

A simple way to obtain the response function analogously to the Gaussian setting (2.18) is by setting

$$
K_{i}(t)=\exp \left(-b_{i}\left(\alpha+\beta t^{2}\right)\right) .
$$

Note that by extrapolation to $q=0$, this would imply

$$
\rho(x)=S(x, 0)=\rho(x) \int_{S^{2}} \psi(x, u) d u,
$$


and therefor a scaling behavior of the fiber density $\psi$ : presence of multiple fibers in a voxel leads to each of them having a smaller value of $\psi$. While this can be seen as sensible given that $\psi$ is a density, this behavior is neither observed in deconvolution reconstructions unless (2.20) is explicitly enforced, not is it desirable since it violates smoothness assumptions of density functions along fibers.

Another option instead of (2.19) is to allow for different tensor eigenvalues on the different shells,

$$
K_{i}(t)=\exp \left(-b_{i}\left(\alpha_{i}+\beta_{i} t^{2}\right)\right)
$$

Other work in this direction includes multi-shell Q-Ball imaging [ALS+10], and linear models like [DDL+01] or [ATBo9] that differ in the choice of the radial basis.

\subsubsection{Discussion}

For the rest of this thesis, we will focus on linear forward models that act as identity on the spatial part,

$$
T \psi(x, q)=T_{0}(\psi(x, \cdot))(q),
$$

encompassing single- and multi-shell deconvolution or more general $q$-space sampling strategies, Tuch's Q-Ball model and the solid-angle Q-Ball model if treating $\log (-\log (S / \rho))$ as given data, and other linear models like the multi-shell models cited above. A number of non-linear models have also been suggested, like Persistent Angular Structure [JAo3] and Diffusion Orientation Transform [ÖSV+o6]. No model currently in use seems to fulfill all requirements regarding physical justification and numerical tractability.

Note that in most cases, DW-MRI reconstructions are carried out in a voxel-by-voxel way, splitting the 5 -dimensional problem on $\mathbb{R}^{3} \times S^{2}$ into a series of independent problems on $S^{2}$. Since the main aim of this thesis is to develop a spatial regularization strategy which takes into account the spatial coherence of the resulting ODFs or fiber densities, this approach will not be possible anymore. This justifies restricting to linear forward models in order to avoid excessive computational complexity.

$\psi$ will from now on be called ODF even when talking about fiber densities. If no confusion arises, the spatial variable will sometimes be omitted.

\subsubsection{Generalized scalar measures}

The ADC and FA measures for diffusion tensors introduced above can be generalized to the multi-fiber case. This was first introduced by Tuch in [Tuco4]. Given an ODF $\psi: S^{2} \rightarrow \mathbb{R}$, its generalized ADC is simply its mean value,

$$
\langle\psi\rangle:=\frac{1}{4 \pi} \int_{S^{2}} \psi(u) d u .
$$


The generalized FA is then defined as

$$
\operatorname{GFA}(\psi):=\left(\frac{\int_{S^{2}}(\psi(u)-\langle\psi\rangle)^{2} d u}{\int_{S^{2}} \psi(u)^{2} d u}\right)^{\frac{1}{2}} .
$$

In general, an ODF for Gaussian diffusion with diffusion tensor $D$ fulfills $\langle\psi\rangle \neq\langle D\rangle$ and $\operatorname{GFA}(\psi) \neq \mathrm{FA}(D)$. These measures are therefore only conceptually related, not strict generalizations. 



\section{Elements from Regularization Theory}

This chapter gives an outline of some results from regularization theory, starting with some general terminology and known results on linear inverse problems in Section 3.1. Particular focus is put on Tikhonov regularization for linear ill-posed problems, as this is the framework employed in the following chapters. In Section 3.2, a convergence result is proved for Tikhonov regularization with a convex constraint and including discretization of the forward operator.

\subsection{Linear Inverse Problems}

Let $X$ and $Y$ be Hilbert spaces and $T: X \rightarrow Y$ a linear bounded operator. Given $y \in Y$, we are interested in determining $x \in X$ such that

$$
T x=y \text {. }
$$

The problem of determining $x$ is called well-posed in the sense of Hadamard if

- there exists a solution

- which is unique and

- which depends continuously on the data.

In more abstract terms, this is the case if and only if $\mathcal{N}(T)=\{0\}, y \in \mathcal{R}(T)$ and $\mathcal{R}(T)$ is closed.

In applications, often some or all of these criteria are not met, in which case the problem is called ill-posed. Existence and uniqueness may fail, for example, due to missing data, such that $\mathcal{N}(T) \neq\{0\}$, or due to data perturbed by inevitable measurement errors, such that $y \notin \mathcal{R}(T)$. The latter case also poses difficulties for solving the problem if $x$ does not depend continuously on $y$, since measurement errors may be arbitrarily amplified. In all these cases, it is desirable to extend the notion of a solution of (3.1). 
Definition 3.1. $x \in X$ is called a least-squares solution of (3.1) if

$$
\|T x-y\|=\inf _{z \in X}\|T z-y\| .
$$

It is called best approximate solution if

$$
\|x\|=\inf \{\|z\|: z \text { is a least-squares solution }\} .
$$

It can be shown (see for example [EHN96]) that best approximate solutions exist if and only if $y \in \mathcal{R}(T)+\mathcal{R}(T)^{\perp}=: \mathcal{D}\left(T^{\dagger}\right)$, defining a linear operator $T^{\dagger}: \mathcal{D}\left(T^{\dagger}\right) \rightarrow \mathcal{N}(T)^{\perp}$, the Moore-Penrose pseudo-inverse of $T$. Throughout this chapter, it is assumed that a best approximate solution

$$
x^{\dagger}:=T^{\dagger} y
$$

to (3.1) in this sense exists.

$T^{\dagger}$ is bounded if and only if $\mathcal{R}(T)$ is closed (cf. [EHN96, Prop. 2.4]). Therefore, given noisy data $y^{\delta}$ close to $y$ in the sense that $\left\|y-y^{\delta}\right\| \leq \delta, x^{\delta}$ given by (3.2) with $y$ replaced by $y^{\delta}$ may not be a good approximation to the true solution $x^{\dagger}$. A regularization method is essentially an approximation $R \simeq T^{\dagger}$ such that $\left\|R y^{\delta}-x^{\dagger}\right\|$ is small. More precisely:

Definition 3.2. Let $T: X \rightarrow Y$ be a bounded linear operator, and $\left(R_{\alpha}\right)_{\alpha \in A}$ with $R_{\alpha}: Y \rightarrow X a$ family of continuous (possibly non-linear) operators parametrized by a set A. A parameter choice rule is a map $\bar{\alpha}:(0, \infty) \times Y \rightarrow A$, and the pair $(R, \bar{\alpha})$ is called a regularization method for $T$ if

$$
\limsup _{\delta \rightarrow 0}\left\{\left\|R_{\bar{\alpha}\left(\delta, y^{\delta}\right)} y^{\delta}-x^{\dagger}\right\|: y^{\delta} \in Y,\left\|T x^{\dagger}-y^{\delta}\right\| \leq \delta\right\}
$$

for all $x^{\dagger} \in \mathcal{N}(T)^{\perp}$.

Convergence of regularization methods can be investigated in terms of the worst case error

$$
\Delta(T, R, M, \delta):=\sup \left\{\left\|R y^{\delta}-x^{\dagger}\right\|: x^{\dagger} \in M, y^{\delta} \in Y,\left\|T x^{\dagger}-y^{\delta}\right\| \leq \delta\right\},
$$

where $M \subset X$ is a subset reflecting some prior knowledge on the solution $x^{\dagger}$. In the general setting however, convergence of regularization methods for ill-posed problems can be arbitrarily slow:

Theorem 3.3. Assume there exists a regularization method $(R, \bar{\alpha})$ and a continuous function $\varphi: \mathbb{R}^{+} \rightarrow \mathbb{R}^{+}$with $\varphi(0)=0$ such that

$$
\Delta\left(T, R_{\bar{\alpha}(\delta, \cdot)}, M, \delta\right) \leq \varphi(\delta)
$$

with

$$
M=\left\{x \in \mathcal{N}(T)^{\perp}:\|T x\| \leq 1\right\} .
$$

Then $T^{\dagger}$ is continuous. 
Proof. Let $\left(y_{n}\right)_{n \in \mathbb{N}} \subset \mathcal{D}\left(T^{\dagger}\right)$ be a sequence with $\left\|y_{n}\right\| \leq 1$ and $y_{n} \rightarrow y \in \mathcal{D}\left(T^{\dagger}\right)$ as $n \rightarrow \infty$. Then

$$
\left\|T^{\dagger} y_{n}-T^{\dagger} y\right\| \leq\left\|T^{\dagger} y_{n}-R_{\bar{\alpha}\left(\delta, y_{n}\right)} y_{n}\right\|+\left\|R_{\bar{\alpha}\left(\delta, y_{n}\right)} y_{n}-T^{\dagger} y\right\| .
$$

Both terms can be estimated using assumption (3.4), the first one by putting $x^{\dagger}=T^{\dagger} y_{n}$ and $y^{\delta}=y_{n}$ in (3.3), the second one by putting $x^{\dagger}=T^{\dagger} y$ and $y^{\delta}=y_{n}$, yielding

$$
\left\|T^{\dagger} y_{n}-T^{\dagger} y\right\| \leq 2 \varphi\left(\left\|y_{n}-y\right\|\right)
$$

This shows that $T^{\dagger} y_{n} \rightarrow T^{\dagger} y$.

Thus, in order to obtain convergence rates, the set of solutions $M$ has to be restricted in some way. The most frequently used way to do this is by employing source conditions.

Definition 3.4. A spectral source condition is a condition of the form

$$
x^{\dagger}=\varphi\left(T^{*} T\right) \omega, \quad \omega \in X,\|\omega\| \leq \rho
$$

for some $\rho>0$ and an index function $\varphi:\left[0,\|T\|^{2}\right] \rightarrow \mathbb{R}$, i.e. $\varphi$ is continuous, strictly increasing and $\varphi(0)=0$. The corresponding source set is

$$
M_{\rho}(\varphi):=\left\{x=\varphi\left(T^{*} T\right) \omega: \omega \in X,\|\omega\| \leq \rho\right\}
$$

Since $T$ is usually a smoothing operator, source conditions can be interpreted as abstract smoothness conditions on the solution. A widely used choice for $\varphi-$ and the only one we will be interested in - are the Hölder type source conditions, where

$$
\varphi(\lambda)=\varphi_{\mu}(\lambda):=\lambda^{\mu}
$$

and $\mu>0$ is called the Hölder exponent.

It is interesting to see if lower bounds on the worst case error can be given in order to assess the optimality of a given regularization method. For Hölder source conditions, it is known that the best attainable convergence rate is

$$
\inf _{R} \Delta\left(T, R, M_{\rho}\left(\varphi_{\mu}\right), \delta_{k}\right) \leq \delta_{k}^{\frac{2 \mu}{2 \mu+1}} \rho^{\frac{1}{2 \mu+1}}
$$

for a series $\delta_{k} \rightarrow 0$. Regularization methods fulfilling this bound are called optimal, while those fulfilling

$$
\Delta\left(T, R_{\bar{\alpha}(\delta, \cdot)}, M_{\rho}\left(\varphi_{\mu}\right), \delta\right) \leq C \delta^{\frac{2 \mu}{2 \mu+1}} \rho^{\frac{1}{2 \mu+1}}
$$

for some $C \geq 1$ are said to be of optimal order. 


\subsubsection{Tikhonov Regularization}

A frequently employed method is Tikhonov regularization. To motivate it, remember that by Definition 3.1, the best approximate solution minimizes the functional $x \mapsto$ $\left\|T x-y^{\delta}\right\|^{2}$. The idea is to replace this functional by

$$
J_{\alpha}(x):=\left\|T x-y^{\delta}\right\|^{2}+\alpha\|x\|^{2},
$$

for $\alpha>0$, i.e. to define the regularization operator by

$$
R_{\alpha} y^{\delta}=\underset{x \in X}{\operatorname{argmin}} J_{\alpha}(x)
$$

The additional term in $J_{\alpha}$ disfavors solutions that are too large.

Due to the following lemma, the method is well-defined.

Lemma 3.5. For every $y^{\delta} \in Y$ and $\alpha>0, J_{\alpha}$ has a unique minimizer $x_{\alpha}^{\delta}$. It is given by

$$
x_{\alpha}^{\delta}=R_{\alpha} y^{\delta}:=\left(T^{*} T+\alpha\right)^{-1} T^{*} y^{\delta} .
$$

$x_{\alpha}^{\delta}$ depends continuously on $y^{\delta}$.

Proof. Uniqueness and the expression (3.8) are shown in [EHN96, Theorem 5.1]. Boundedness of $\left(T^{*} T+\alpha\right)^{-1}$ follows from the Lax-Milgram lemma.

Tikhonov regularization belongs to a class of regularization methods that can be studied using spectral theory, since (3.8) can be written as

$$
R_{\alpha} y^{\delta}=t_{\alpha}\left(T^{*} T\right) T^{*} y^{\delta}
$$

with $t_{\alpha}(\lambda)=(\lambda+\alpha)^{-1}$. Using this, it can be shown that

$$
\left\|R_{\alpha} y^{\delta}-R_{\alpha} y\right\|=\left\|t_{\alpha}\left(T^{*} T\right) T^{*}\left(y^{\delta}-y\right)\right\| \leq \alpha^{-1 / 2}\left\|y^{\delta}-y\right\|
$$

and that under a Hölder source condition with exponent $\mu \leq 1$

$$
\left\|R_{\alpha} T x^{\dagger}-x^{\dagger}\right\|=\left\|\left(1-t_{\alpha}\left(T^{*} T\right) T^{*} T\right)\left(T^{*} T\right)^{\mu} \omega\right\| \leq c \alpha^{\mu}
$$

for some $c>0$. Hence

$$
\left\|R_{\alpha} y^{\delta}-x^{\dagger}\right\| \leq c \rho \alpha^{\mu}+\frac{\delta}{\sqrt{\alpha}}=\mathcal{O}\left(\delta^{\frac{2 \mu}{2 \mu+1}}\right)
$$

if $\alpha \sim \delta^{\frac{2}{2 \mu+1}}$. In particular, Tikhonov regularization is of optimal order for $\mu \leq 1$. 


\subsubsection{Constrained Tikhonov Regularization}

In some cases, one knows in advance that that the true solution $x^{\dagger}$ lies in some convex subset $C \subset X$. In those cases, the result can often be improved by replacing the Tikhonov functional $J_{\alpha}$ in (3.7) with

$$
J_{\alpha}^{C}(x):=\|T x-y\|^{2}+\alpha\|x\|^{2}+\chi_{C}(x),
$$

where

$$
\chi_{C}(x)= \begin{cases}0, & x \in C, \\ \infty, & \text { otherwise }\end{cases}
$$

is the indicator function of the set $C$. Thus, one solves

$$
R_{\alpha, C} y^{\delta}=\underset{x \in C}{\operatorname{argmin}} J_{\alpha}(x)
$$

If $C$ is closed and convex, a minimizer of $J_{\alpha}^{C}$ exists and is uniquely determined (see for example [EHN96, Theorem 5.15]). Since $R_{\alpha, C}$ is non-linear, spectral theory can not be employed in the convergence analysis. Convergence of order $\sqrt{\delta}$ for this method was shown in [Neu88] under the projected source condition

$$
x^{\dagger}=P_{C}\left(T^{*} \omega\right) \text { for some } \omega \in Y,
$$

where $P_{C}: X \rightarrow X$ is the metric projection onto $C$. This condition is similar to a Hölder source condition $x^{\dagger} \in \mathcal{R}\left(\left(T^{*} T\right)^{1 / 2}\right)=\mathcal{R}\left(T^{*}\right)$ with exponent $1 / 2$, but can be significantly weaker depending on the constraint set $C$.

\subsection{Discretization of Linear Inverse Problems}

In practice, the problem (3.1) can only be solved in finite dimensions. Therefore, the continuous formulation has to be discretized. This is done by introducing orthogonal projections $P_{h} \in \mathcal{L}(X)$ and $Q_{h} \in \mathcal{L}(Y)$ with finite dimensional range. Here, $h>0$ is a discretization parameter. Then (3.1) is replaced by

$$
T_{h} x:=Q_{h} T P_{h} x=Q_{h} y \text {. }
$$

This equation is then solved for $x \in \mathcal{R}\left(P_{h}\right)$ by the methods introduced in the previous sections. The aim of this section is to investigate the convergence of discretized Tikhonov regularization under a convex constraint.

In the following, it is assumed that $\left\|T_{h}-T\right\| \rightarrow 0$ as $h \rightarrow 0$. To check this property in concrete cases, the following lemma may be useful. 
Lemma 3.6. The following statements are equivalent:

1. $\left\|T_{h}-T\right\| \rightarrow 0$ as $h \rightarrow 0$

2. $\left\|T\left(1-P_{h}\right)\right\| \rightarrow 0$ and $\left\|\left(1-Q_{h}\right) T\right\| \rightarrow 0$

3. $T$ is compact, $P_{h} \rightarrow 1$ pointwise on $\mathcal{R}\left(T^{*}\right)$ and $Q_{h} \rightarrow 1$ pointwise on $\mathcal{R}(T)$

Proof. (1.) implies (2.) since

$$
\left\|\left(1-Q_{h}\right) T\right\| \leq\left\|T_{h}-Q_{h} T\right\|+\left\|T_{h}-T\right\| \leq 2\left\|T_{h}-T\right\|,
$$

and similarly for $\left\|T\left(1-P_{h}\right)\right\|$. The reverse implication holds due to

$$
\left\|T_{h}-T\right\| \leq\left\|\left(1-Q_{h}\right) T P_{h}\right\|+\left\|T\left(1-P_{h}\right)\right\| .
$$

For the rest, note that due to $\left\|T\left(1-P_{h}\right)\right\|=\left\|\left(1-P_{h}\right) T^{*}\right\|$, it suffices to show the statements for $Q_{h}$. The implication (2.) $\Rightarrow$ (3.) holds since $T$ is the limit of finite-dimensional operators $Q_{h} T$ and hence compact, and since $Q_{h} y \rightarrow y$ for all $y=T x$ with $x \in X$. To see the reverse, assume that $\left\|\left(1-Q_{h}\right) T\right\| \rightarrow 0$. Then there are $c>0$ and sequences $\left(h_{n}\right)_{n \in \mathbb{N}} \subset \mathbb{R}$ and $\left(x_{n}\right)_{n \in \mathbb{N}} \subset X$ with $h_{n} \rightarrow 0,\left\|x_{n}\right\|=1$ and

$$
\left\|\left(1-Q_{h_{n}}\right) T x_{n}\right\| \geq c .
$$

By compactness, we can assume without loss of generality that $T x_{n} \rightarrow y \in \overline{\mathcal{R}(T)}$. Then the previous inequality implies that $\left(1-Q_{h}\right) y \rightarrow 0$. On the other hand, uniform boundedness of $\left(Q_{h}\right)_{h>0}$ implies that $Q_{h} \rightarrow 1$ pointwise also on $\overline{\mathcal{R}(T)}$, which is a contradiction.

We cite the following convergence result for the discretized solution under Hölder source conditions in the unconstrained case from [PV90].

Theorem 3.7. Let $P_{h}, Q_{h}$ and $T_{h}$ be as above, $y \in \mathcal{R}(T)$ and $y^{\delta} \in Y$ with $\left\|y-y^{\delta}\right\| \leq \delta$. Assume that $\left\|T-T_{h}\right\| \rightarrow 0$ as $h \rightarrow 0$, and that the regularization parameter $\alpha>0$ is chosen such that

$$
\alpha \rightarrow 0, \quad \frac{\delta^{2}}{\alpha} \rightarrow 0, \quad \frac{\left\|T\left(1-P_{h}\right)\right\|^{2}}{\alpha} \leq c
$$

as $\delta, h \rightarrow 0$ for some $c>0$. Denote by $\bar{x} \in \mathcal{R}\left(P_{h}\right)$ the discrete Tikhonov-regularized solution fulfilling

$$
\left(T_{h}^{*} T_{h}+\alpha\right) \bar{x}=T_{h}^{*} y^{\delta}
$$

Then

$$
\lim _{\delta, h \rightarrow 0} \bar{x}=x^{\dagger}
$$

If, in addition,

$$
x^{\dagger} \in \mathcal{R}\left(\left(T^{*} T\right)^{\mu}\right)
$$


for some $0<\mu \leq \frac{1}{2}$, and

$$
c_{1} \alpha \leq \delta^{\frac{2}{2 \mu+1}}+\left\|T\left(1-P_{h}\right)\right\|^{2} \leq c_{2} \alpha
$$

for some $c_{1}, c_{2}>0$, then

$$
\left\|\bar{x}-x^{\dagger}\right\|=\mathcal{O}\left(\delta^{\frac{2 \mu}{2 \mu+1}}+\left\|T-T_{h}\right\|^{2 \mu}\right)
$$

as $\delta, h \rightarrow 0$.

Unfortunately, the proof in [PV9o] relies on spectral theory and therefore does not generalize to the constrained setting.

To address this problem, spectral theory can be replaced by variational techniques in regularization theory developed in the last decade. They were first introduced in [HKPSo7] for problems with non-linear, non-smooth operators, but also turned out useful to handle more general data fidelities and penalties as well as constrained problems. In the following, we will prove a convergence result similar to Theorem 3.7 using these techniques.

Even though we are only interested in the Tikhonov case, the convergence theorem will be presented in a more abstract way, for which some additional notation is needed. Assume that we are given an ideal data fidelity functional $\mathcal{T} \equiv \mathcal{T}_{y^{+}}: Y \rightarrow[0, \infty]$ with

$$
\mathcal{T}(y)=0 \quad \Longleftrightarrow \quad y=y^{\dagger},
$$

and an empirical data fidelity functional $S \equiv S_{y^{\delta}}: Y \rightarrow \mathbb{R} \cup\{\infty\}$, both convex, which are connected by the error assumption

$$
\frac{1}{A} \mathcal{T}(y)-\varepsilon_{\delta} \leq \mathcal{S}(y)-S\left(y^{\dagger}\right) \leq A\left(\mathcal{T}(y)+\varepsilon_{\delta}\right) \quad \forall y \in Y
$$

for some $A \geq 1$ and $\varepsilon_{\delta}>0$ such that $\varepsilon_{\delta} \rightarrow 0$ as $\delta \rightarrow 0$. The convex regularization penalty is denoted by $\mathcal{R}: X \rightarrow \mathbb{R} \cup\{\infty\}$, and the discretization error is controlled by the assumption

$$
\frac{1}{B} \mathcal{T}(T x)-\eta_{h} \mathcal{R}(x) \leq \mathcal{T}\left(T_{h} x\right) \leq B\left(\mathcal{T}(T x)+\eta_{h} \mathcal{R}(x)\right) \quad \forall x \in C \subset X
$$

for $B \geq 1$ and $\eta_{h}>0$ with $\eta_{h} \rightarrow 0$ as $h \rightarrow 0$, and where $C \subset X$ is the convex constraint set. Then (generalized) discrete constrained Tikhonov regularization consists in solving the optimization problem

$$
\bar{x} \in \underset{x \in C}{\operatorname{argmin}}\left(S\left(T_{h} x\right)+\alpha \mathcal{R}(x)\right)
$$


In the variational formalism, convergence is usually investigated in terms of the Bregman distance of the convex regularization penalty. It is defined as

$$
\mathcal{D}_{\mathcal{R}}\left(x, x^{\dagger}\right) \equiv \mathcal{D}_{\mathcal{R}}^{\xi^{\dagger}}\left(x, x^{\dagger}\right)=\mathcal{R}(x)-\mathcal{R}\left(x^{\dagger}\right)-\left\langle\xi^{\dagger}, x-x^{\dagger}\right\rangle,
$$

where $\xi^{\dagger} \in \partial \mathcal{R}\left(x^{\dagger}\right)$ is a fixed element in the subgradient of $\mathcal{R}$ at $x^{\dagger}$. Note that in general, $\mathcal{D}_{\mathcal{R}}$ is neither symmetric nor does it satisfy a triangle inequality.

An important part in the variational approach consists in replacing the spectral source condition (3.5) by a condition in the form of a variational inequality.

Definition 3.8. $x^{\dagger} \in X$ is said to satisfy an (additive) variational smoothness assumption if there are $\beta>0$ and a concave index function $\psi: \mathbb{R}^{+} \rightarrow \mathbb{R}$ with

$$
\beta \mathcal{D}_{\mathcal{R}}\left(x, x^{\dagger}\right) \leq \mathcal{R}(x)-\mathcal{R}\left(x^{\dagger}\right)+\psi(\mathcal{T}(T x)) \quad \forall x \in C
$$

Quadratic Tikhonov regularization is the special case

$$
\begin{gathered}
\mathcal{T}(y)=\frac{1}{2}\left\|y-y^{\dagger}\right\|^{2}, \\
\mathcal{S}(y)=\frac{1}{2}\left\|y-y^{\delta}\right\|^{2}, \text { and } \\
\mathcal{R}(x)=\frac{1}{2}\|x\|^{2}+\chi_{C}(x) .
\end{gathered}
$$

In this case, assuming that $x^{\dagger} \in C$, the Bregman distance at $x \in C$ is simply

$$
\mathcal{D}_{\mathcal{R}}\left(x, x^{\dagger}\right)=\frac{1}{2}\left\|x-x^{\dagger}\right\|^{2}+\chi_{C}(x) .
$$

To show that (3.12) is fulfilled case with $A=2, B=1$ and

$$
\varepsilon_{\delta}=\left\|y^{\delta}-y^{\dagger}\right\|^{2} \quad \text { and } \quad \eta_{h}=\left\|T-T_{h}\right\|^{2},
$$

we use the inequality $\|a+b\|^{2} \leq 2\|a\|^{2}+2\|b\|^{2}$ to obtain

$$
\frac{1}{2} \mathcal{T}(y)-\varepsilon_{\delta} \leq \frac{1}{2}\left\|y-y^{\delta}\right\|^{2}-\frac{1}{2}\left\|y^{\dagger}-y^{\delta}\right\|^{2}=S(y)-S\left(y^{\dagger}\right)
$$

and

$$
S(y)-S\left(y^{\dagger}\right) \leq\left\|y-y^{\dagger}\right\|^{2}+\frac{1}{2}\left\|y^{\delta}-y^{\dagger}\right\|^{2} \leq 2\left(\mathcal{T}(y)+\varepsilon_{\delta}\right) .
$$

The inequality (3.12a) follows from

$$
\begin{aligned}
\mathcal{T}\left(T_{h} x\right) & =\frac{1}{2}\left\|T_{h} x-y^{\dagger}\right\|^{2} \\
& \leq\left\|T x-y^{\dagger}\right\|^{2}+\left\|\left(T-T_{h}\right) x\right\|^{2} \\
& \leq\left\|T x-y^{\dagger}\right\|^{2}+\left\|T-T_{h}\right\|^{2}\|x\|^{2} \\
& =\mathcal{T}(T x)+\eta_{h} \mathcal{R}(x) .
\end{aligned}
$$


The inequality $\mathcal{T}(T x) \leq \mathcal{T}\left(T_{h} x\right)+\eta_{h} \mathcal{R}(x)$ is obtained in the same way by exchanging $T$ and $T_{h}$.

Several results on the relations between spectral source conditions and variational smoothness assumptions have been obtained (an overview is given in [Fle13]). The following is from [HY10].

Theorem 3.9. In the quadratic Tikhonov case (3.15) and for a linear operator $T: X \rightarrow Y$, the Hölder source condition (3.5) with $\varphi=\varphi_{\mu}$ given by (3.6) for $0<\mu \leq \frac{1}{2}$ implies (3.14) with $\psi \sim \varphi_{\nu}$ for $\nu=\frac{2 \mu}{2 \mu+1}$ and $\beta \in(0,1)$ arbitrary.

For larger Hölder exponents, this theorem can not hold. In fact, in [HY1o] it is also proved that (3.14) with $\psi \sim \varphi_{\nu}$ for $\nu>1 / 2$ implies $x^{\dagger}=0$. So in this sense, variational smoothness assumptions only cover Hölder source conditions with $\mu \leq 1 / 2$.

Moreover, in [FH11], equivalence between the projected source condition (3.9) and (3.14) with $\psi(t) \sim \sqrt{t}$ was shown. This serves as the main motivation to approach the discrete, constrained problem with these methods.

To prove the convergence theorem, some basic results from convex analysis are required:

Definition 3.10. Given a convex, lower semi-continuous function $f: \mathbb{R}^{n} \rightarrow \mathbb{R} \cup\{\infty\}$ not constantly $\infty$, its conjugate function $f^{*}$ is defined by

$$
f^{*}(y)=\sup _{x \in \mathbb{R}^{n}}\left(y^{T} x-f(x)\right), \quad s \in \mathbb{R} .
$$

Iff is only defined on a subset of $\mathbb{R}^{n}$, it is understood to be extended to all of it by $\infty$.

Lemma 3.11. The following statements hold:

- $f(x)+f^{*}(y) \geq y^{T} x$

- $f(x)+f^{*}(y)=y^{T} x \Longleftrightarrow y \in \partial f(x) \Longleftrightarrow x \in \partial f^{*}(y)$, and

- $f^{* *}:=\left(f^{*}\right)^{*}=f$.

Proof. More general versions of these statements are shown for example in [ET76, Chapter 1].

Theorem 3.12. Let the relations (3.12) hold and $\bar{x}$ be defined as the assumed to be unique minimizer in (3.13). Assume further that $x^{\dagger}$ is the unique $\mathcal{R}$-minimizing solution to Tx $=y^{\dagger}$. 
1. Let $\mathcal{T}$ and $\mathcal{R}$ be weakly lower semi-continuous and the sets $\{x \in X: \mathcal{R}(x) \leq c\}$ be weakly sequentially compact for all $c>0 .^{1}$ If $\alpha$ is chosen such that $\alpha \rightarrow 0$ and

$$
\frac{1}{\alpha}\left(\mathcal{S}\left(T_{h} x^{\dagger}\right)-\inf _{x \in C} S\left(T_{h} x\right)\right) \rightarrow 0
$$

as $\delta, h \rightarrow 0$, then

$$
\lim _{\delta, h \rightarrow 0} \mathcal{D}_{\mathcal{R}}\left(\bar{x}, x^{\dagger}\right)=0
$$

2. If the variational smoothness assumption (3.14) holds true, then

$$
\beta \mathcal{D}_{\mathcal{R}}\left(\bar{x}, x^{\dagger}\right) \leq A B \psi(\text { err })
$$

with

$$
\boldsymbol{e r r}=\left(A B+A^{-1}\right) \mathcal{R}\left(x^{\dagger}\right) \eta_{h}+(1+A) \varepsilon_{\delta},
$$

if $\alpha$ is chosen suitably (see (3.19) below).

Proof. By definition,

$$
\mathcal{S}\left(T_{h} \bar{x}\right)+\alpha \mathcal{R}(\bar{x}) \leq \mathcal{S}\left(T_{h} x^{\dagger}\right)+\alpha \mathcal{R}\left(x^{\dagger}\right)
$$

and thus, using (3.16),

$$
\limsup _{\delta, h \rightarrow 0} \mathcal{R}(\bar{x}) \leq \mathcal{R}\left(x^{\dagger}\right) .
$$

We now show that each sequence of minimizers $\left(\bar{x}_{k}\right)_{k \in \mathbb{N}}$ fulfilling the requirements with corresponding errors $\varepsilon_{k}, \eta_{k} \rightarrow 0$ has a subsequence that converges weakly to $x^{\dagger}$. This then implies that $\bar{x} \rightarrow x^{\dagger}$ as $\delta, h \rightarrow 0$.

Due to (3.18) and the compactness assumption on $\mathcal{R},\left(\bar{x}_{k}\right)$ has a weakly convergent subsequence $\bar{x}_{k(l)} \rightarrow \tilde{x}$ as $l \rightarrow \infty$. Then $T \bar{x}_{k(l)} \rightarrow \tilde{T} x$, since every continuous linear operator is weakly continuous. Using the weak lower semi-continuity of $\mathcal{T},(3.17)$ and the error relations (3.12), we obtain

$$
\begin{aligned}
\mathcal{T}(T \tilde{x}) & \leq \liminf _{l \rightarrow \infty} \mathcal{T}\left(T \bar{x}_{k(l)}\right) \\
& \leq \liminf _{l \rightarrow \infty} B\left(\mathcal{T}\left(T_{k(l)} \bar{x}_{k(l)}\right)+\eta_{h} \mathcal{R}(x)\right) \\
& \leq \liminf _{l \rightarrow \infty} B \mathcal{T}\left(T_{k(l)} \bar{x}_{k(l)}\right) \\
& \leq \liminf _{l \rightarrow \infty} A B\left(S_{k(l)}\left(T_{k(l)} \bar{x}_{k(l)}\right)-S_{k(l)}\left(y^{\dagger}\right)\right) \\
& \leq \liminf _{l \rightarrow \infty} A B\left(S_{k(l)}\left(T_{k(l)} x^{\dagger}\right)-S_{k(l)}\left(y^{\dagger}\right)+\alpha_{k(l)}\left(\mathcal{R}\left(x^{\dagger}\right)-\mathcal{R}\left(\bar{x}_{k(l)}\right)\right)\right) \\
& =\liminf _{l \rightarrow \infty} A B\left(S_{k(l)}\left(T_{k(l)} x^{\dagger}\right)-S_{k(l)}\left(y^{\dagger}\right)\right) \\
& \leq \liminf _{l \rightarrow \infty} A^{2} B \mathcal{T}\left(T_{k(l)} x^{\dagger}\right) \\
& \leq A^{2} B^{2} \mathcal{T}\left(T x^{\dagger}\right) \\
& =0
\end{aligned}
$$

${ }^{1}$ Both assumptions are fulfilled in the quadratic Tikhonov case if the constraint set $C$ is weakly closed. 
It follows that $T \tilde{x}=y^{\dagger}$. Uniqueness of $x^{\dagger}$ and $\mathcal{R}(\tilde{x}) \leq \mathcal{R}\left(x^{\dagger}\right)$ then implies that $\tilde{x}=x^{\dagger}$.

Therefore, as claimed, $\bar{x} \rightarrow x^{\dagger}$. In particular, $\left\langle\tilde{\zeta}^{\dagger}, \bar{x}-x^{\dagger}\right\rangle \rightarrow 0$. Due to (3.18),

$$
\mathcal{D}_{\mathcal{R}}\left(\bar{x}, x^{\dagger}\right)=\mathcal{R}(\bar{x})-\mathcal{R}\left(x^{\dagger}\right)-\left\langle\xi^{\dagger}, \bar{x}-x^{\dagger}\right\rangle \rightarrow 0 .
$$

This shows the first part.

For the second part, let $\gamma \geq 0$ be arbitrary. The variational smoothness assumption, the inequality (3.17) and the fact that $\bar{x} \in C$ yield

$$
\begin{aligned}
\beta \mathcal{D}_{\mathcal{R}}\left(\bar{x}, x^{\dagger}\right) & \leq \mathcal{R}(\bar{x})-\mathcal{R}\left(x^{\dagger}\right)+\psi(\mathcal{T}(T \bar{x})) \\
& =\gamma\left(\mathcal{R}\left(x^{\dagger}\right)-\mathcal{R}(\bar{x})\right)+(1+\gamma)\left(\mathcal{R}(\bar{x})-\mathcal{R}\left(x^{\dagger}\right)\right)+\psi(\mathcal{T}(T \bar{x})) \\
& \leq \gamma\left(\mathcal{R}\left(x^{\dagger}\right)-\mathcal{R}(\bar{x})\right)+\frac{1+\gamma}{\alpha}\left(\mathcal{S}\left(T_{h} x^{\dagger}\right)-\mathcal{S}\left(T_{h} \bar{x}\right)\right)+\psi(\mathcal{T}(T \bar{x})) .
\end{aligned}
$$

Using (3.12), we obtain

$$
\begin{aligned}
\mathcal{S}\left(T_{h} x^{\dagger}\right)-\mathcal{S}\left(T_{h} \bar{x}\right) & =\mathcal{S}\left(T_{h} x^{\dagger}\right)-\mathcal{S}\left(y^{\dagger}\right)+\mathcal{S}\left(y^{\dagger}\right)-\mathcal{S}\left(T_{h} \bar{x}\right) \\
& \leq A\left(\mathcal{T}\left(T_{h} x^{\dagger}\right)+\varepsilon_{\delta}\right)-\frac{1}{A} \mathcal{T}\left(T_{h} \bar{x}\right)+\varepsilon_{\delta} \\
& \leq A B \mathcal{R}\left(x^{\dagger}\right) \eta_{h}+(1+A) \varepsilon_{\delta}-\frac{1}{A B} \mathcal{T}(T \bar{x})+\frac{\eta_{h}}{A} \mathcal{R}(\bar{x}) .
\end{aligned}
$$

We now choose $\gamma$ such that

$$
\frac{\eta_{h}}{A \alpha}(1+\gamma)=\gamma
$$

to make $\mathcal{R}(\bar{x})$-terms vanish. Note that we must have $A \alpha>\eta_{h}$ for $\gamma$ to be non-negative, which will however be ensured by the choice for $\alpha$ below. We arrive at

$$
\beta \mathcal{D}_{\mathcal{R}}\left(\bar{x}, x^{\dagger}\right) \leq \frac{1+\gamma}{\alpha} \text { err }-\frac{1+\gamma}{A B \alpha} \mathcal{T}(T \bar{x})+\psi(\mathcal{T}(T \bar{x}))
$$

By definition of the conjugate function of $-\psi$, this can be estimated further by

$$
\beta D_{\mathcal{R}}\left(\bar{x}, x^{\dagger}\right) \leq \frac{1+\gamma}{\alpha} \text { err }+(-\psi)^{*}\left(-\frac{1+\gamma}{A B \alpha}\right) .
$$

Setting $s:=-(1+\gamma)(A B \alpha)^{-1}$, the infimum of the right hand side is

$$
\inf _{s<0}\left(-s A B \text { err }+(-\psi)^{*}(s)\right)=-(-\psi)^{* *}(A B \text { err })=\psi(A B \text { err }) \leq A B \psi(\text { err }) .
$$

Due to the second statement in Lemma 3.11, it is attained if

$$
s \in \partial(-\psi)(A B \text { err })
$$

which is equivalent to

$$
A \alpha \in \eta_{h}+(-B \partial(-\psi)(A B \text { err }))^{-1} .
$$

In particular, since $\psi$ is strictly increasing, $A \alpha>\eta_{h}$ as needed above. 
Remark 3.13. 1. In the unconstrained case, $\inf _{x \in X} S\left(T_{h} x\right)=\frac{1}{2}\left\|\left(1-Q_{h}\right) y^{\delta}\right\|^{2}$ is attained if $T_{h} x=Q_{h} y^{\delta}$. Therefore, by the characterization of the best approximation as orthogonal projection,

$$
\begin{aligned}
\mathcal{S}\left(T_{h} x^{\dagger}\right)-\inf _{x \in \mathcal{X}} \mathcal{S}\left(T_{h} x\right) & =\frac{1}{2}\left\|Q_{h} T P_{h} x^{\dagger}-Q_{h} y^{\delta}\right\|^{2} \\
& \leq\left\|Q_{h}\left(y^{\delta}-y^{\dagger}\right)\right\|^{2}+\left\|Q_{h} T\left(1-P_{h}\right) x^{\dagger}\right\|^{2} \\
& \leq\left\|y^{\delta}-y^{\dagger}\right\|^{2}+\left\|T\left(1-P_{h}\right)\right\|^{2}\left\|\left(1-P_{h}\right) x^{\dagger}\right\|^{2} .
\end{aligned}
$$

Hence, (3.16) is implied by the conditions (3.10) of Theorem 3.7.

2. Since the explicit form $T_{h}=Q_{h} T P_{h}$ was not used anywhere but just the fact that $\left\|T-T_{h}\right\| \rightarrow 0$, the theorem actually gives an error estimate for more general operator errors. These may occur for example due to uncertainties in modeling the forward operator. Thus, the theorem can also be viewed as a way to partially alleviate some of the modeling issues discussed in Chapter 2.

3. Using the assumptions of Theorem 3.7 together with Theorem 3.9, we have shown a convergence rate

$$
\left\|\bar{x}-x^{\dagger}\right\|=\mathcal{O}\left(\left(\delta+\left\|T-T_{h}\right\|\right)^{\frac{2 \mu}{2 \mu+1}}\right),
$$

which is identical to the one in (3.11) with respect to the measurement error both are of optimal order - , but somewhat worse with respect to the operator error.

4. Since the proof does not rely on linearity of $T$ and $T_{h}$, it actually also holds in the non-linear case. A very similar result - with almost identical proof - can be found in [LF12, Theorem 3.1], where convergence is shown under the assumption

$$
\eta_{h} \sim \sup _{x \in C}\left\|T(x)-T_{h}(x)\right\|^{2} \rightarrow 0
$$

for data fidelity terms given by a Banach space norm. Compared to that result, an error estimate of the form (3.12), despite having been introduced here merely as a notation, has been shown to be applicable in much more general settings than error assumptions based on norm-distances (cf. [HW13]). Moreover, (3.12) also holds in the linear case for unbounded constraint sets $C$, when (3.20) is typically not satisfied. 


\section{A Penalty for ODFs}

\subsection{Choice of the Regularization Functional}

In Chapter 3, Tikhonov regularization was introduced as a way to incorporate prior knowledge into the reconstruction, essentially by requiring that the norm of the reconstruction be sufficiently small. It is clear that in this regard the space $X$ of possible solutions has to be chosen appropriately in order to achieve good reconstruction results. For example, in order to enforce smoothness of the solutions, one might take $X$ to be the Sobolev space $H^{1}$ of weakly differentiable functions.

For ODF reconstruction, this choice is usually too restrictive, since it promotes isotropic smoothness of the reconstruction, i.e. in each spatial direction. ODFs on the other hand can only be assumed to be smooth along the fibers, but not perpendicular to them.

In [Duio5], Durrs introduced a formalism for smoothing a related class of functions, namely functions on $\mathbb{R}^{2} \times S^{1}$, with the aim to enhance elongated and crossing structures. The idea is to identify $\mathbb{R}^{2} \times S^{1}$ with the two-dimensional Euclidean group SE(2) of translations and rotations. The group structure then provides

- a distinct class of curves in $\mathrm{SE}(2)$, the exponential curves parametrized by a starting point in $\mathrm{SE}(2)$ and a starting tangential vector, that can be viewed as generalizations of straight lines to SE(2) and serve as a local model for elongated structures, and

- a set of derivatives along these curves called left-invariant derivatives.

These derivatives are then used to construct non-linear diffusion filters on SE(2) by taking an image as initial state $\left.\psi\right|_{t=0}: \operatorname{SE}(2) \rightarrow \mathbb{R}$ of a diffusion equation

$$
\partial_{t} \psi=\nabla_{L}^{T} D[\psi] \nabla_{L} \psi,
$$

where $\nabla_{L}$ is the left-invariant gradient and the diffusion tensor $D[\psi]: \operatorname{SE}(2) \rightarrow \mathbb{R}^{3 \times 3}$ is adapted to the local structure of the image. This equation is then solved up to some stopping time $t=\tau$. The resulting image $\left.\psi\right|_{t=\tau}$ can be seen as a denoised and enhanced version of the original image. The result depends crucially on the way $D[\psi]$ is constructed from $\psi$. In general, one tries to direct diffusion along elongated structures in order to enhance coherence, but not perpendicular to them in order to preserve edges. Doing this relies on estimating the orientation of the local structure, 
which is done by finding the exponential curve that locally fits best to the image, in some appropriate sense.

In [DF11], DuITs and FRANKEn generalize their formalism to the three-dimensional case, i.e. to functions on $\mathbb{R}^{3} \times S^{2}$, and apply this to the enhancement of HARDI reconstructions. The main challenge here is the fact that $\mathbb{R}^{3} \times S^{2}$ can not be identified with $\operatorname{SE}(3)$, the latter being isomorphic to $\mathbb{R}^{3} \ltimes \mathrm{SO}(3)$. Instead, $\mathbb{R}^{3} \times S^{2}$ can only be identified with a quotient of $\mathrm{SE}$ (3) by a non-normal subgroup isomorphic to $\mathrm{SO}(2)$ and therefore does not inherit a group structure. To carry over concepts from the $\mathbb{R}^{2} \times S^{1}$-case, one has to require that they are well-defined on the quotient.

The constructed diffusion filters are employed as a post-processing step, after reconstructing the ODF from DW-MRI data. Our aim is to include concepts from this formalism directly in the reconstruction algorithm by formulating a suitable penalty functional that can be used in for Tikhonov regularization.

In the following, a short overview of the $S E$ (3)-formalism, in particular of the construction of exponential curves, will be given.

Definition 4.1. The Euclidean Motion Group SE(n) in $n$ dimensions is the semi-direct product $\mathbb{R}^{n}$ and the group $\mathrm{SO}(n)$ of rotations in $n$ dimensions,

$$
\mathrm{SE}(n):=\mathbb{R}^{n} \rtimes \mathrm{SO}(n),
$$

i.e. $\mathrm{SE}(n)=\mathbb{R}^{n} \times \mathrm{SO}(n)$ as a set, with the product between $g=(b, R)$ and $g^{\prime}=\left(b^{\prime}, R^{\prime}\right)$ given by $g g^{\prime}=\left(b+R b^{\prime}, R R^{\prime}\right)$. The unit element is $e=(0, \mathbb{1})$ and the inverse of $g$ is $g^{-1}=$ $\left(-R^{-1} b, R^{-1}\right)$. SE $(n)$ acts on $\mathbb{R}^{n}$ as group of rotations and translations by $g x:=R x+b$ for $x \in \mathbb{R}^{n}$.

A matrix representation of $\mathrm{SE}(n)$ is given by

$$
\mathrm{SE}(n) \ni(b, R) \mapsto\left(\begin{array}{cc}
R & b \\
0 & 1
\end{array}\right) \in \mathbb{R}^{(n+1) \times(n+1)} .
$$

$\operatorname{SE}(n)$ acts in a natural way on $\mathbb{R}^{n} \times S^{n-1}$ by

$$
(b, R)(x, u):=(R x+b, R u),
$$

i.e. by translating the spatial part and simultaneously rotating the spatial and the orientational parts. By identifying

$$
\mathbb{R}^{n} \times S^{n-1} \ni(x, u) \mapsto\left(\begin{array}{cc}
u & x \\
0 & 1
\end{array}\right) \in \mathbb{R}^{(n+1) \times 2},
$$

this action is given simply by matrix multiplication. Moreover, the tangent spaces to $\mathbb{R}^{n} \times S^{n-1}$ can be identified with matrices of the form

$$
T_{(x, u)}\left(\mathbb{R}^{n} \times S^{n-1}\right) \simeq T_{x} \mathbb{R}^{n} \times T_{u} S^{n-1} \ni\left(X_{x}, X_{u}\right) \mapsto\left(\begin{array}{cc}
X_{u} & X_{x} \\
0 & 0
\end{array}\right) \in \mathbb{R}^{(n+1) \times 2},
$$


and with this identification, the derivative of the group action in (4.1) is again simply given by matrix multiplication.

We are particularly interested in the case $n=3$. For this, it is convenient to introduce some additional notation:

Definition 4.2. For $a \in \mathbb{R}^{3}$, denote by $a^{\times} \in \mathbb{R}^{3 \times 3}$ the skew-symmetric matrix that acts as $a^{\times} x=a \times x$, i.e. the vector product between $a$ and $x$, for $x \in \mathbb{R}^{3}$.

For all $a, b \in \mathbb{R}^{3}$ and $R \in \mathrm{SO}(3)$, one has

- $a^{\times} b^{\times}=b a^{T}-\left(b^{T} a\right) \mathbb{1}$,

- $\left(a^{\times} b\right)^{\times}=b a^{T}-a b^{T}=a^{\times} b^{\times}-b^{\times} a^{\times}$, and

- $(R a)^{\times}=R a^{\times} R^{T}$.

Having identified $\mathrm{SE}(n)$ with a subgroup of $\mathrm{Gl}(n+1, \mathbb{R})$, one can prove the following theorem.

Theorem 4.3. SE $(n)$ is a Lie Group. For $n=3$, its Lie Algebra $\mathfrak{s e ( 3 )}$ consists of all matrices of the form

$$
\mathfrak{s e}(3) \ni\left(b, a^{\times}\right) \equiv\left(\begin{array}{cc}
a^{\times} & b \\
0 & 0
\end{array}\right) \in \mathbb{R}^{4 \times 4},
$$

for $a, b \in \mathbb{R}^{3}$, and the Lie bracket is given by the commutator $\left[X_{1}, X_{2}\right]=X_{1} X_{2}-X_{2} X_{1}$. More explicitly,

$$
\left[\left(b_{1}, a_{1}^{\times}\right),\left(b_{2}, a_{2}^{\times}\right)\right]=\left(a_{1} \times b_{2}+b_{1} \times a_{2},\left(a_{1} \times a_{2}\right)^{\times}\right) .
$$

$\mathfrak{s e}(n)$ is isomorphic to the space of left-invariant vector fields on $\mathrm{SE}(3)$, i.e. vector fields ${ }^{1}$ $V \in \Gamma(T S E(3))$ fulfilling $V(g h)=g V(h)$ for all $g, h \in \mathrm{SE}(3)$, the isomorphism being

$$
\mathfrak{s e}(n) \ni X \mapsto(g \mapsto g X) \in \Gamma(\operatorname{TSE}(3)) .
$$

Proof. See [Bako2].

As already mentioned above, $\mathrm{SE}(3)$ is larger than the domain $\mathbb{R}^{3} \times S^{2}$ on which ODFs are defined. The two spaces are related by a projection $\pi_{\xi}: \operatorname{SE}(3) \rightarrow \mathbb{R}^{3} \times S^{2}$ which is constructed by choosing an arbitrary

$$
\xi \equiv\left(0, \xi_{0}\right) \in \mathbb{R}^{3} \times S^{2}
$$

and defining

$$
\pi_{\xi}(x, R):=(x, R) \xi=\left(x, R \xi_{0}\right) .
$$

${ }^{1} \Gamma$ (TSE(3)) denotes the space of smooth sections of the tangent bundle of $\mathrm{SE}(3)$, i.e. functions $V: \mathrm{SE}(3) \rightarrow T \mathrm{SE}(3)$ such that for all $g \in \mathrm{SE}(3), V(g)$ is in the tangential space $T_{g} \mathrm{SE}(3)$ of $\mathrm{SE}(3)$ at $g$. 
The kernel of this projection is the stabilizer

$$
S_{\xi}=\{h \in \mathrm{SE}(3): h \xi=\xi\}=\left\{(0, R) \in \mathrm{SE}(3): R \xi_{0}=\xi_{0}\right\} \simeq \mathrm{SO}(2) .
$$

$\pi_{\xi}$ is surjective, so $\mathbb{R}^{3} \times S^{2}$ can be identified with the coset space $\mathrm{SE}(3) / S_{\tilde{\xi}}$. Note that the stabilizer can also be written as

$$
S_{\xi}=\left\{\left(0, \exp \left(s \xi_{0}^{\times}\right)\right): s \in \mathbb{R}\right\}=\left\{\exp \left(s\left(0, \xi_{0}^{\times}\right)\right): s \in \mathbb{R}\right\} .
$$

Moreover, $X \in \mathfrak{s e}(3)$ fulfills $X \xi=0$ if and only if $X=t\left(0, \xi_{0}^{\times}\right)$for some $t \in \mathbb{R}$.

In [DF11], a right inverse to $\pi_{\tilde{\xi}}$, i.e. and embedding of $\mathbb{R}^{3} \times S^{2}$ into $\mathrm{SE}(3)$, is constructed using Euler angles on $\mathrm{SO}(3)$ and spherical coordinates on $S^{2}$. Unfortunately, this embedding is ill-defined at the coordinate poles. More generally, there is no such (continuous) embedding: if $\iota_{\xi}: \mathbb{R}^{3} \times S^{2} \rightarrow \mathrm{SE}(3)$ fulfilled $\pi_{\xi} \circ \iota_{\xi}(p)=p$ for all $p$, restricting it to $\{0\} \times S^{2}$ would yield a continuous map $\rho: S^{2} \rightarrow \mathrm{SO}(3)$ such that $\rho(u) \xi_{0}=u$ for all $u \in S^{2}$. Now take a vector $\eta_{0} \perp \xi_{0}$. Then $\rho(u) \eta_{0} \perp u$, so $u \mapsto \rho(u) \eta_{0}$ is a non-vanishing, continuous tangential vector field on $S^{2}$, contradicting the hairy ball theorem ${ }^{2}$.

An important role in the SE(3)-formalism is played by exponential curves.

Definition 4.4. Let $g \in \mathrm{SE}(3)$ and $X \in \mathfrak{s e}(3)$. The curve $\gamma$ fulfilling

$$
\gamma^{\prime}(t)=\gamma(t) X, \quad \gamma(0)=g
$$

is called exponential curve through $g$ with parameter X.

Using the matrix representation of $\mathfrak{s e}(3)$, exponential curves are simply given using the matrix exponential as

$$
\gamma(t)=g \exp (t X)
$$

The simplest way to construct exponential curves on the quotient is as follows: given $(x, u) \in \mathbb{R}^{3} \times S^{2}$ and $X \in \mathfrak{s e}(3)$, take $g \in \pi_{\xi}^{-1}(x, u)$, construct the exponential curve (4.2) and project it back to $\mathbb{R}^{3} \times S^{2}$ using $\pi_{\xi}$. The question is: under what circumstances does the resulting curve not depend on the arbitrary choice of $g$ and $\xi$ ? For this to hold, we must have

$$
g h \exp (t X) \xi=g \exp (t X) \xi
$$

for all $t \in \mathbb{R}$ and $h \in S_{\xi}$. Writing this as

$$
\exp (-t X) \exp \left(s\left(0, \xi_{0}^{\times}\right)\right) \exp (t X) \xi=\xi
$$

for $s \in \mathbb{R}$, differentiating by $s$ and $t$ and setting $s=t=0$ shows that

$$
\left[\left(0, \xi_{0}^{\times}\right), X\right] \xi=0 .
$$

\footnotetext{
${ }^{2}$ The hairy ball theorem states that every continuous, tangential vector field on $S^{2}$ has a zero.
} 
With $X=\left(b, a^{\times}\right)$, this can be expanded as

$$
\left(\begin{array}{cc}
\left(\xi_{0} \times a\right) \times \xi_{0} & \xi_{0} \times b \\
0 & 0
\end{array}\right)=0
$$

so $a, b \in \operatorname{span}\left\{\xi_{0}\right\}$. Now

$$
\pi_{\xi}\left(g \exp \left(\left(t \xi_{0}, s \xi_{0}^{\times}\right)\right)\right)=\pi_{\xi}\left(g \exp \left(t\left(\xi_{0}, 0\right)\right)\right)=(x+t u, u)
$$

is the horizontal line, which is therefore the only well-defined exponential curve on the quotient.

On the other hand, the construction above may have been too restrictive, since $X \in \mathfrak{s e}(3)$ and $g \in \pi_{\xi}^{-1}(x, u)$ were chosen fixed and independent of each other. A possible way to improve the situation is to choose the parameter $X$ of the exponential curve depending on the choice of $g$, i.e. to consider

$$
\mathbb{R} \ni t \mapsto g \exp (t X(g)) \xi
$$

for $X: \pi_{\xi}^{-1}(x, u) \rightarrow \mathfrak{s e}(3)$. For this to be well-defined, we need

$$
\begin{aligned}
& g h \exp (t X(g h)) \xi=g \exp (t X(g)) \xi \\
& \Longleftrightarrow \quad h \exp (t X(g h)) h^{-1} \xi=\exp (t X(g)) \xi
\end{aligned}
$$

for all $h \in S_{\xi}$. Differentiating by $t$ and setting $t=0$ yields

$$
\operatorname{Ad}_{h} X(g h) \xi=X(g) \xi
$$

where $\operatorname{Ad}_{h} X:=h X h^{-1}$ is the adjoint representation. Explicitly, $\operatorname{Ad}_{h}\left(b, a^{\times}\right)=\left(R b,(R a)^{\times}\right)$ for $h=(0, R)$. In particular, $\operatorname{Ad}_{h}\left(0, \xi_{0}^{\times}\right)=\left(0, \xi_{0}^{\times}\right)$for all $h \in S_{\xi}$.

Define an inner product on $\mathfrak{s e}(3)$ by

$$
\left\langle\left(b_{1}, a_{1}^{\times}\right),\left(b_{2}, a_{2}^{\times}\right)\right\rangle:=a_{1}^{T} a_{2}+b_{1}^{T} b_{2} .
$$

Then $\operatorname{Ad}_{h}$ leaves span $\left\{\left(0, \xi_{0}^{\times}\right)\right\}$and its orthogonal complement invariant. $X(g)$ can be uniquely decomposed as $X(g)=X_{\perp}(g)+c(g)\left(0, \xi_{0}^{\times}\right)$for some $c: \pi_{\xi}^{-1}(x, u) \rightarrow \mathbb{R}$ and $X_{\perp}(g) \perp\left(0, \xi_{0}^{\times}\right)$. Then equation (4.6) implies that

$$
\operatorname{Ad}_{h} X_{\perp}(g h)=X_{\perp}(g) .
$$

Putting this back into (4.5), we obtain the requirement

$$
\exp \left(t X_{\perp}(g)+t c(g h)\left(0, \xi_{0}^{\times}\right)\right) \xi=\exp \left(t X_{\perp}(g)+t c(g)\left(0, \xi_{0}^{\times}\right)\right) \xi .
$$

Differentiating twice with respect to $t$, setting $t=0$ and using $\left(0, \xi_{0}^{\times}\right) \xi=0$ leads to

$$
(c(g h)-c(g))\left(0, \xi_{0}^{\times}\right) X_{\perp}(g) \xi=0 .
$$


Write $X_{\perp}$ as $X_{\perp}(g)=\left(b(g), a(g)^{\times}\right)$, with $a(g) \perp \xi_{0}$ by construction, and assume first that

$$
\left(0, \xi_{0}^{\times}\right) X_{\perp}(g) \xi=\left(\begin{array}{cc}
a(g) & \xi_{0} \times b(g) \\
0 & 0
\end{array}\right)=0 .
$$

Then $a(g)=0$ and $b(g)=b_{0} \xi_{0}$, with $b_{0}$ being constant due to (4.7). Hence, in this case, $X(g)=c(g) \xi_{0}^{\times}+b_{0} \xi_{0}$, and the projected curve (4.4) is again the horizontal line, which does not depend on $c$ at all. So with no loss one may take $X(g)=b_{0} \xi_{0}$. If, on the other hand, $\left(0, \xi_{0}^{\times}\right) X_{\perp}(g) \xi \neq 0$, then $c$ has to be constant. This observation and equation (4.7) can be summarized by requiring that, without loss of generality, $X$ has to fulfill

$$
\operatorname{Ad}_{h} X(g h)=X(g) \quad \text { for all } h \in S_{\xi}
$$

in order for (4.4) to be well-defined.

Condition (4.8) has a nice interpretation. First, note that $\pi_{\xi}^{-1}(x, u) \ni g \mapsto g X(g)$ is a vector field tangential to $\mathrm{SE}(3)$. Composing this with the differential of the projection $\pi_{\xi}$ yields a function $g \mapsto g X(g) \xi \in T_{(x, u)}\left(\mathbb{R}^{3} \times S^{2}\right)$. Since

$$
g h X(g h) \xi=g X(g) h \xi=g X(g) \xi,
$$

this function is in fact constant. Therefore, every $X: \pi_{\tilde{\xi}}^{-1}(x, u) \rightarrow \mathfrak{s e}(3)$ fulfilling (4.8) determines a tangential vector $\pi_{(x, u)}^{*} X \in T_{(x, u)}\left(\mathbb{R}^{3} \times S^{2}\right) . \pi_{(x, u)}^{*}$ is surjective and $\mathcal{N}\left(\pi_{(x, u)}^{*}\right)=\operatorname{span}\left\{g \mapsto\left(0, \xi_{0}^{\times}\right)\right\}$. Thus, the space of functions fulfilling (4.8) can be interpreted as the tangent space $T_{(x, u)}\left(\mathbb{R}^{3} \times S^{2}\right)$ plus one additional dimension.

An inverse to $\pi_{(x, u)}^{*}$ can be given explicitly: if $\pi_{(x, u)}^{*} X=\left(V_{x}, V_{u}\right)$, then

$$
X(x, R)=\left(R^{T} V_{x},\left(R^{T}\left(u \times V_{u}+c u\right)\right)^{\times}\right)
$$

for some $c \in \mathbb{R}$, where $R \in \mathrm{SO}(3)$ with $R \xi_{0}=u$. Finally, the corresponding projected exponential curve $g \exp (t X(g)) \xi=\left(\gamma_{x}(t), \gamma_{u}(t)\right)$ has the explicit form

$$
\begin{gathered}
\gamma_{x}(t)=x+\frac{\left(W \cdot V_{x}\right) W}{\|W\|^{2}} t-\frac{W \times\left(W \times V_{x}\right)}{\|W\|^{3}} \sin (\|W\| t)+\frac{W \times V_{x}}{\|W\|^{2}}(1-\cos (\|W\| t)), \\
\gamma_{u}(t)=\frac{(W \cdot u) W}{\|W\|^{2}}-\frac{W \times(W \times u)}{\|W\|^{2}} \cos (\|W\| t)+\frac{W \times u}{\|W\|} \sin (\|W\| t),
\end{gathered}
$$

with $W=u \times V_{u}+c u$ if $W \neq 0$, and

$$
\gamma_{x}(t)=x+V_{x} t, \quad \gamma_{u}(t)=u,
$$

otherwise. The spatial part is a helix with axis $W$, while the orientational part performs the corresponding rotation that keeps its components constant with respect to the moving frame of the spatial part. In particular, the curves actually depend on $c \in$ $\mathbb{R}$, so the space of exponential curves at $(x, u)$ is larger than the tangential space $T_{(x, u)}\left(\mathbb{R}^{3} \times S^{2}\right)$. 
In [Frao8], orientation estimation in $\mathrm{SE}(2)$ is done by introducing left-invariant derivatives, which are essentially derivatives along exponential curves. Since these curves are parametrized by elements in $\mathfrak{s e}(2)$, the left-invariant gradient $\nabla_{L} \psi$ of a function $\psi: \mathrm{SE}(2) \rightarrow \mathbb{R}$ can be interpreted as a map from $\mathrm{SE}(2)$ to the fixed space $\mathfrak{s e}(2)^{*}$. Therefore, it is possible to take second derivatives, which is in general not possible for the usual derivative. In particular, it is possible to compute the left-invariant Hessian matrix. The local orientation is then taken as the (right) singular vector corresponding to the smallest singular value. ${ }^{3}$ This is motivated by the requirement that $\nabla_{L} \psi$ should change as little as possible along the exponential curve corresponding to the local orientation $X(g)$ at $g \in \mathrm{SE}(2)$ :

$$
X(g)=\underset{\|Y\|=1}{\operatorname{argmin}}\left\|\left.\partial_{t} \nabla_{L} \psi\left(g \gamma_{Y}(t)\right)\right|_{t=0}\right\| .
$$

When applying this to our case, several problems arise:

- There are too many exponential curves: they are parametrized by a six-dimensional space, while ODFs are only defined on $\mathbb{R}^{3} \times S^{2}$. Intuitively, it seems clear that local knowledge of an ODF can not determine a unique exponential curve. Indeed, the left-invariant Hessian in this case is singular with left singular vector $\xi_{0}^{\times}$. The interpretation of the corresponding right singular vector is not clear. Moreover, numerical experiments showed frequent cases in which there is more than one small singular value; in these cases it is not clear which singular value is the "trivial" one and should be discarded.

- On the other hand, restricting the space of allowed curves in the most obvious way - namely putting $c=0$ in the definition of $W$ above - does not seem reasonable: it restricts the spatial part $\gamma_{x}$ of the curve to be a helix with axis perpendicular to the orientational part $u$ of the starting point $(x, u) \in \mathbb{R}^{3} \times S^{2}$. In addition to being a rather arbitrary choice, it also has the undesirable effect that it is impossible for all points in $\{x\} \times U \subset \mathbb{R}^{3} \times S^{2}$ with $U \subset S^{2}$ an arbitrarily small, open set to belong to the same oriented structure except for a straight line, $W=0$.

- A more sensible approach might be to restrict some intrinsic parameters of the projected exponential curve. For example, one might assume that the spatial part of the curve is torsion-free. The torsion of $\gamma_{x}$ can be explicitly calculated to be $\tau=W \cdot V_{x}$, leading to an additional indefinite quadratic constraint in the $\mathrm{SE}$ (3)-version of (4.9), which is therefore more complicated and time-intensive to solve, and may not even have a unique solution.

- In order to estimate local orientation, a (small) minimization problem like (4.9) has to be solved at each point of a discretization of $\mathbb{R}^{3} \times S^{2}$. While this may be acceptable when solving a diffusion equation as a post-processing step, for example by computing one low-dimensional SVD at each point for each time

${ }^{3}$ Using singular vectors instead of eigenvectors is necessary since the Hessian is in general nonsymmetric since the left-invariant derivatives are non-commutative. 
step of an explicit time-discretization, computation cost can quickly become prohibitive when employed in an inversion algorithm.

- Finally, it is not clear how to formulate a penalty for Tikhonov regularization that is related to the non-linear diffusion used by Duits and Franken.

Since generalization of Hessian-based non-linear diffusion to $\mathbb{R}^{3} \times S^{2}$ does not seem possible in a straight-forward way, in [DCGD11] the authors resort to two basic approaches. Firstly, they consider linear diffusion filters where diffusion predominantly occurs along the horizontal line (4.3). In the following, we will call the derivative along this line the horizontal derivative (see also Definition 4.8 below):

$$
D_{\mathrm{hor}} \psi(x, u):=\left.\partial_{t} \psi(x+t u, u)\right|_{t=0}=u \cdot \operatorname{grad} \psi(x, u)
$$

for a smooth function $\psi: \mathbb{R}^{3} \times S^{2} \rightarrow \mathbb{R}$, where grad is the gradient with respect to the spatial variable $x$. Then linear diffusion amounts to solving

$$
\partial_{t} \psi=c_{\mathrm{hor}} D_{\mathrm{hor}}^{2} \psi+c_{\perp}\left(\Delta_{\mathbb{R}^{3}}-D_{\mathrm{hor}}^{2}\right) \psi+c_{S^{2}} \Delta_{S^{2}} \psi
$$

for some constants $c_{\text {hor }}, c_{S^{2}}>0$ and $c_{\perp} \geq 0$ with $c_{\text {hor }} \gg c_{\perp}$. Using the intuition above, this amounts to assuming that, without any further knowledge of the image, the best guess for the local orientation at $(x, u) \in \mathbb{R}^{3} \times S^{2}$ is simply a line in direction $u$, while allowing for some curvature as controlled by the parameter $c_{S^{2}}$.

As an extension to this, non-linear, adaptive diffusion is done by replacing the $D_{\text {hor }}$-term above with

$$
c_{\text {hor }} D_{\text {hor }} \exp \left(-\kappa\left|D_{\text {hor }} \psi\right|^{2}\right) D_{\text {hor }} \psi
$$

with $\kappa>0$, thus stopping diffusion at edges, where $D_{\text {hor }} \psi$ is large. The exponential function may also be replaced by other non-negative, decreasing functions. Non-linear diffusion of this type is related to Tikhonov regularization with penalty functionals of the form (cf. [SGG+o9, Chapter 6])

$$
\psi \mapsto \int_{\mathbb{R}^{3} \times S^{2}} \Phi\left(\left|D_{\text {hor }} \psi(x, u)\right|^{2}\right) d x d u .
$$

In the following, we will focus on the linear case and use a penalty functionals of the form

$$
\psi \mapsto\|\psi\|^{2}+\left\|D_{\text {hor }} \psi\right\|^{2}
$$

and

$$
\psi \mapsto\|\psi\|^{2}+\left\|D_{\text {hor }} \psi\right\|^{2}+\left\|\operatorname{grad}_{S^{2}} \psi\right\|^{2} .
$$

A formal justification for the inclusion of the angular derivative as well as a more rigorous description of this penalty will be developed in the next section. 
Remark 4.5. In[RK11], REISERT and KISELEV describe a very similar method of spatial regularization based on a penalty of the form

$$
\psi \mapsto\left\|D_{\text {hor }} \psi\right\|^{2} .
$$

Although this regularization scheme leads to good results, its theoretical properties have not been studied yet. In particular, compactness properties, which are needed to establish convergence of discrete approximations (Theorem 3.12), are not obvious since the forward operator is not smoothing in the spatial part. As was observed in the work cited above, and will also be confirmed in the numerical experiments in Chapter 6, this spatial regularization method has characteristic weaknesses in regions with non-straight elongated structures. The modified method (4.12) is able to to better cope with some of these situations.

\subsection{The Space $H_{\text {hor }}\left(\Omega \times S^{2}\right)$}

For the remainder of this section,

$$
\Omega:=\prod_{i=1}^{3}\left(-\frac{L_{i}}{2}, \frac{L_{i}}{2}\right),
$$

where $L \in \mathbb{R}_{+}^{3}$, is a cube in $\mathbb{R}^{3}$. The simplify the notation, all function spaces will be understood to be defined on $\Omega \times S^{2}$ unless stated otherwise.

Since the spatial part of the DW-MRI forward operator is essentially a Fourier transform and inversion algorithms usually employ FFTs in some way or another, it is natural to take trigonometric polynomials as spatial basis functions, and therefore use function spaces with periodic boundary conditions. This is not a severe restriction in practice since in many cases the support of the object under consideration does not touch the boundary of the volume.

Definition 4.6. The space $C_{\text {per }}^{\infty}$ of smooth, spatially periodic functions on $\Omega \times S^{2}$ is defined by restriction as

$$
C_{\text {per }}^{\infty}:=\left\{\left.f\right|_{\Omega \times S^{2}}: f \in C^{\infty}\left(\mathbb{R}^{3} \times S^{2}\right), f\left(\cdot+n^{T} L, \cdot\right)=\text { f for all } n \in \mathbb{Z}^{3}\right\} .
$$

As basis functions on $\Omega$, we use trigonometric functions

$$
\xi_{k}(x)=|\Omega|^{-\frac{1}{2}} \prod_{j=1}^{3} \exp \left(2 \pi i L_{j}^{-1} k_{j} x_{j}\right), \quad k \in \mathbb{Z}^{3},
$$

while on $S^{2}$, Spherical Harmonics (SHs) are used. They are defined as

$$
Y_{l m}(u)=\sqrt{\frac{2 l+1}{4 \pi} \frac{(l-m) !}{(l+m) !}} P_{l}^{m}\left(u_{3}\right)\left(\frac{u_{1}+i u_{2}}{\left|u_{1}+i u_{2}\right|}\right)^{m}
$$


for $l \in \mathbb{N}_{0}$ and $m \in \mathbb{Z},|m| \leq l$. Here $P_{l}^{m}$ are the associated Legendre functions. For details on the SHs, see e.g. [Nédo1]. Then $\left\{\eta_{k l m}\right\}_{k l m}$ with $\eta_{k l m}:=\xi_{k} \otimes Y_{l m}$ is an orthonormal basis of $L^{2}$.

The tangential surface gradient of a smooth function $f: S^{2} \rightarrow \mathbb{R}$ is given by $\operatorname{Grad} f(u):=$ $\left(1-u u^{T}\right) \nabla f(u)$ for $u \in S^{2}$. Here, $\nabla f$ is the gradient of an arbitrary smooth extension of $f$ to a neighborhood of $S^{2} \subset \mathbb{R}^{3}$. It can be shown that the result does not depend on the choice of this extension due to the projection $1-u u^{T}$. We denote by

$$
\operatorname{Grad}_{i} f:=e_{i}^{T} \operatorname{Grad} f
$$

the components of the gradient operator, where $e_{i}$ for $i=1,2,3$ is the standard basis of $\mathbb{R}^{3}$.

The Laplace-Beltrami operator on $S^{2}$ is defined as $\Delta_{S^{2}}=$ Div $\circ$ Grad, where the divergence is Div $=-$ Grad $^{*}$. Explicit expressions in spherical coordinates can be found e.g. in [Nédo1]. Using these, it can be shown that $\Delta_{S^{2}} u_{i}=-2 u_{i}$. Therefore,

$$
\begin{gathered}
\int_{S^{2}} \operatorname{Grad}_{i} f(u) d u=\int_{S^{2}}\left(\operatorname{Grad} u_{i}\right)^{T} \operatorname{Grad} f(u) d u \\
=-\int_{S^{2}}\left(\Delta_{S^{2}} u_{i}\right) f(u) d u=2 \int_{S^{2}} u_{i} f(u) d u .
\end{gathered}
$$

Hence

$$
\int_{S^{2}} f(u) \operatorname{Grad}_{i} g(u) d u=\int_{S^{2}}\left(-\operatorname{Grad}_{i} f(u)+2 u_{i} f(u)\right) g(u) d u .
$$

So, formally, the adjoint of $\mathrm{Grad}_{i}$ is

$$
\operatorname{Grad}_{i}^{*} f(u)=-\operatorname{Grad}_{i} f(u)+2 u_{i} f(u) .
$$

The adjoint Grad $^{*}=-$ Div relates to the components $\operatorname{Grad}_{i}^{*}$ by

$$
\operatorname{Grad}^{*} V(u)=\sum_{i} \operatorname{Grad}_{i}^{*} V_{i}(u)=-\sum_{i} \operatorname{Grad}_{i} V_{i}(u)
$$

for a smooth tangential vector field $V$ on $S^{2}$, i.e. $V: S^{2} \rightarrow \mathbb{R}^{3}$ with $u^{T} V(u)=0$ for all $u \in S^{2}$.

In the following, we use the notation Grad $\psi$ for $\psi \in C_{\mathrm{per}}^{\infty}$ to denote the derivative with respect to the angular variables, while grad $\psi$ denotes the spatial derivative:

Definition 4.7. For $\psi \in L^{2}$, define

$$
\operatorname{grad} \psi:=\sum_{k l m}\left\langle\psi, \eta_{k l m}\right\rangle\left(\operatorname{grad} \xi_{k}\right) \otimes Y_{l m}
$$

and

$$
\operatorname{Grad} \psi:=\sum_{k l m}\left\langle\psi, \eta_{k l m}\right\rangle \xi_{k} \otimes\left(\operatorname{Grad} Y_{l m}\right),
$$

if the series converge in $\left(L^{2}\right)^{3}$. 
The inner product here is to be taken in $L^{2}$; from here on, this will always be case, unless otherwise indicated. The same holds for norms, i.e.

$$
\|\cdot\| \equiv\|\cdot\|_{L^{2}}, \quad\langle\cdot, \cdot\rangle \equiv\langle\cdot, \cdot\rangle_{L^{2}} .
$$

Additionally, for the next definition and thereafter, it will be convenient to denote the function $\Omega \times S^{2} \rightarrow S^{2},(x, u) \mapsto u$ simply by $u$.

Definition 4.8. The horizontal derivative of a function $\psi \in L^{2}$ is defined as

$$
D_{\text {hor }} \psi:=\sum_{k l m}\left\langle\psi, \eta_{k l m}\right\rangle \sum_{i} \frac{\partial \xi_{k}}{\partial x_{i}} \otimes\left(u_{i} Y_{l m}\right),
$$

if the series converges in $L^{2}$.

The horizontal space $H_{\text {hor }}$ is defined as the space of all $\psi \in L^{2}$ for which

$$
\|\psi\|_{\text {hor }}^{2}:=\|\psi\|^{2}+\left\|D_{\text {hor }} \psi\right\|^{2}+\|\operatorname{Grad} \psi\|^{2}<\infty .
$$

$D_{\text {hor }} \psi=\varphi$ holds if and only if

$$
\langle\varphi, g\rangle=-\left\langle\psi, D_{\text {horg }} g\right\rangle \quad \text { for all } g \in C_{\text {per }}^{\infty} .
$$

This can be seen by first noting that for $g, h \in C_{\text {per }}^{\infty},\left\langle D_{\text {hor }} g, h\right\rangle=-\left\langle g, D_{\text {hor }} h\right\rangle$, and that $\eta_{k l m} \in C_{\text {per. }}^{\infty}$ Thus

$$
\left\langle D_{\text {hor }} \psi, g\right\rangle=\sum_{k l m}\left\langle\psi, \eta_{k l m}\right\rangle\left\langle D_{\text {hor }} \eta_{k l m}, g\right\rangle=-\left\langle\psi, D_{\text {hor }} g\right\rangle
$$

On the other hand, if (4.16) hold true, then

$$
\begin{aligned}
\left\langle\varphi, \eta_{k l m}\right\rangle & =-\sum_{k^{\prime} l^{\prime} m^{\prime}}\left\langle\psi, \eta_{k^{\prime} l^{\prime} m^{\prime}}\right\rangle\left\langle\eta_{k^{\prime} l^{\prime} m^{\prime}}, D_{\text {hor }} \eta_{k l m}\right\rangle \\
& =\left\langle\sum_{k^{\prime} l^{\prime} m^{\prime}}\left\langle\psi, \eta_{k^{\prime} l^{\prime} m^{\prime}}\right\rangle D_{\text {hor }} \eta_{k^{\prime} l^{\prime} m^{\prime}}, \eta_{k l m}\right\rangle .
\end{aligned}
$$

(4.16) implies that $D_{\text {hor }}$ is closed, since if $\psi_{n} \rightarrow \psi$ and $D_{\text {hor }} \psi_{n} \rightarrow \varphi$ in $L^{2}$, then

$$
\langle\varphi, g\rangle=\lim _{n \rightarrow \infty}\left\langle D_{\text {hor }} \psi_{n}, g\right\rangle=-\lim _{n \rightarrow \infty}\left\langle\psi_{n}, D_{\text {hor }} g\right\rangle=-\left\langle\psi, D_{\text {hor }} g\right\rangle,
$$

so $\varphi=D_{\text {hor }} \psi$.

For notational purposes, we define the operator $D_{0}: H_{\text {hor }} \subset L^{2} \rightarrow\left(L^{2}\right)^{3} \times\left(L^{2}\right)^{3}$ by

$$
D_{0}:=\left(\begin{array}{c}
D_{\text {hor }} \\
\text { Grad }
\end{array}\right)
$$

in order to write

$$
\|\psi\|_{\text {hor }}^{2}=\|\psi\|^{2}+\left\|D_{0} \psi\right\|^{2}
$$


Lemma 4.9. $H_{\text {hor }}$ equipped with the norm $\|\cdot\|_{\text {hor }}$ is a Hilbert space. It coincides with the domain of the self-adjoint operator $\left|D_{0}\right|:=\left(D_{0}^{*} D_{0}\right)^{1 / 2}$.

Proof. The closedness of both $D_{\text {hor }}$ and Grad implies that $D_{0}$ is closed. Therefore, its domain $\mathcal{D}\left(D_{0}\right)=H_{\text {hor }}$ is a Hilbert space with the graph norm $\|\cdot\|_{\text {hor }}=\left(\|\cdot\|^{2}+\left\|D_{0} \cdot\right\|^{2}\right)^{\frac{1}{2}}$.

Self-adjointness of $D_{0}^{*} D_{0}$ (initially defined for example on $C^{\infty}$ ) can be established by a Friedrichs extension (see e.g. [Tay96, Appendix A.8]). Hence $\left|D_{0}\right|$ is well defined by the functional calculus. By the polar decomposition of closed operators in Hilbert spaces we have $\mathcal{D}\left(\left|D_{0}\right|\right)=\mathcal{D}\left(D_{0}\right)$.

$C_{\text {per }}^{\infty}$ is dense in $H_{\text {hor }}$. This can be shown using the following lemma.

Lemma 4.10. Let $H$ be a Hilbert space, $D: \mathcal{D}(D) \subset H \rightarrow H$ and $T_{n}: H \rightarrow H$ be linear, self-adjoint operators, $X \subset H$ a subspace with $\mathcal{R}\left(T_{n}\right) \subset X \subset \mathcal{D}\left(D^{2}\right)$ and assume that $T_{n} \psi \rightarrow \psi$ for all $\psi \in H$. If there exists a subspace $\Phi \subset \mathcal{D}\left(D^{2}\right)$ that is dense in $H$ such that

$$
D^{2} T_{n} \varphi \rightarrow D^{2} \varphi \quad \text { for all } \varphi \in \Phi,
$$

then $X$ is dense in $\mathcal{D}(D)$ with respect to the graph norm $\|\cdot\|_{D}^{2}:=\|\cdot\|_{H}^{2}+\|D \cdot\|_{H}^{2}$.

Proof. In a first step, we show that $X$ is dense in $\mathcal{D}\left(D^{2}\right)$. Let $\psi \in \mathcal{D}\left(D^{2}\right)$. Using the pointwise convergence of $T_{n}$,

$$
\left\langle D T_{n} \psi, \varphi\right\rangle_{H} \rightarrow\langle D \psi, \varphi\rangle_{H^{\prime}}
$$

and by density of $\Phi$ it follows that $D T_{n} \psi \rightarrow D \psi$. Similarly, (4.17) implies that

$$
\left\langle T_{n} D^{2} T_{n} \psi, \varphi\right\rangle_{H}=\left\langle T_{n} \psi, D^{2} T_{n} \varphi\right\rangle_{H} \rightarrow\left\langle\psi, D^{2} \varphi\right\rangle_{H}=\left\langle D^{2} x, \varphi\right\rangle_{H}
$$

$\varphi$ can be replaced with $\psi$ here by density, i.e.

$$
\left\|D T_{n} \psi\right\| \rightarrow\|D \psi\|
$$

Since weak convergence and convergence of the norms imply strong convergence,

$$
D T_{n} \psi \rightarrow D \psi
$$

follows. This shows that $X$ is dense in $\mathcal{D}\left(D^{2}\right)$ with respect to $\|\cdot\|_{D}$.

The general case $\psi \in \mathcal{D}(D)$ can be reduced to the special case above by density of $\mathcal{D}\left(D^{2}\right)$ in $\mathcal{D}(D)$ shown below: For $\psi \in \mathcal{D}(D)$ and $\varepsilon>0$ there exists $\psi_{\varepsilon} \in \mathcal{D}\left(D^{2}\right)$ such that $\left\|\psi-\psi_{\varepsilon}\right\|_{D} \leq \varepsilon / 2$ and $n \in \mathbb{N}$ such that $\left\|\psi_{\varepsilon}-T_{n} \psi_{\varepsilon}\right\|_{D} \leq \varepsilon / 2$. Hence, $\left\|\psi-T_{n} \psi_{\varepsilon}\right\|_{D} \leq \varepsilon$.

The density of $\mathcal{D}\left(D^{2}\right)$ in $\mathcal{D}(D)$ can be shown by considering the spectral decomposition $\left(E_{\lambda}\right)_{\lambda \in \mathbb{R}}$ of $D$. For $\psi \in \mathcal{D}(D)$, set

$$
\psi_{n}:=E_{n} \psi-E_{-n} \psi, \quad n \in \mathbb{N} .
$$


Then

$$
\left\|D^{2} \psi_{n}\right\|_{H}^{2}=\int_{-\infty}^{\infty} \lambda^{4} d\left\|E_{\lambda} \psi_{n}\right\|_{H}^{2}=\int_{-n-}^{n+} \lambda^{4} d\left\|E_{\lambda} x\right\|_{H}^{2} \leq n^{4}\|\psi\|_{H^{\prime}}^{2}
$$

i.e. $\psi_{n} \in \mathcal{D}\left(D^{2}\right)$. Moreover, $\psi_{n} \rightarrow \psi$ and $D \psi_{n}=E_{n} D \psi-E_{-n} D \psi \rightarrow D \psi$.

We can now prove the density result.

Lemma 4.11. $C_{\text {per }}^{\infty}$ and $\operatorname{span}\left\{\eta_{k l m}\right\}$ are dense in $H_{\text {hor }}$.

Proof. Since $\operatorname{span}\left\{\eta_{k l m}\right\} \subset C_{\text {per }}^{\infty}$, it suffices to consider $\operatorname{span}\left\{\eta_{k l m}\right\}$.

In the preceding lemma, take $H=L^{2}, X=\Phi=C_{\text {per }}^{\infty}$ and $D=\left|D_{0}\right|$. Note that $\|\cdot\|_{D}=\|\cdot\|_{\text {hor }}$ and $\mathcal{D}(D)=\mathcal{D}\left(D_{0}\right)=H_{\text {hor }}$. The operators $T_{n}$ are defined as

$$
T_{n} \psi:=\sum_{k l m}^{n}\left\langle\psi, \eta_{k l m}\right\rangle \eta_{k l m}
$$

where the sum runs over all $(k, l, m)$ with $\|k\| \leq n,|l| \leq n$ and $|m| \leq l$. Then $\mathcal{R}\left(T_{n}\right) \subset X$ and $T_{n} \psi \rightarrow \psi$ in $H$ as $n \rightarrow \infty$.

Condition (4.17) follows using $D^{2}=D_{0}^{*} D_{0}=-\Delta_{S^{2}}-D_{\text {hor }}^{2}$ from

$$
\Delta_{S^{2}} T_{n} \varphi=T_{n} \Delta_{S^{2}} \varphi \rightarrow \Delta_{S^{2}} \varphi
$$

and

$$
D_{\text {hor }}^{2} T_{n} \varphi=\sum_{i, j=1}^{3} u_{i} u_{j}\left(T_{n} \partial_{x_{i}} \partial_{x_{j}} \varphi\right) \rightarrow \sum_{i=1}^{3} u_{i} u_{j} \partial_{x_{i}} \partial_{x_{j}} \varphi=D_{\text {hor }}^{2} \varphi,
$$

where we used that $\Delta_{S^{2}} \varphi$ and $\partial_{x_{i}} \partial_{x_{j}} \varphi$ are in $H$ for $\varphi \in \Phi$, and that both spatial and angular derivatives commute with $T_{n}$.

We will also need Sobolev spaces on $\Omega \times S^{2}$, which can be defined as follows.

Definition 4.12. Define the operator $\Lambda^{s}$ by

$$
\Lambda^{s} \psi:=\sum_{k l m} \lambda_{k l}^{s}\left\langle\psi, \eta_{k l m}\right\rangle \eta_{k l m}
$$

where $\lambda_{k l}=\sqrt{1+\|k\|^{2}+l^{2}}$, whenever the series converges in $L^{2}$. The Sobolev space $H^{s}$ of order $s \geq 0$ is then defined as the set of all $\psi \in L^{2}$ for which

$$
\Lambda^{s} \psi \in L^{2},
$$

equipped with the inner product

$$
\langle\psi, \varphi\rangle_{H^{s}}:=\left\langle\Lambda^{s} \psi, \Lambda^{s} \varphi\right\rangle
$$


Note that the notation $\Lambda^{s}$ is justified, since it is indeed a power of $\Lambda:=\Lambda^{1}$. We will only be interested in the spaces $H^{s}$ for $s=1 / 2$ and $s=1$.

Lemma 4.13. $H^{s}$ is a Hilbert space.

Proof. The proof is analogous to the one of Lemma 4.9.

Using $\operatorname{grad}_{i} \xi_{k}=2 \pi i k_{i} L_{i}^{-1} \xi_{k}$ and $\left\langle\operatorname{Grad} Y_{l m^{\prime}}, \operatorname{Grad} Y_{l^{\prime} m^{\prime}}\right\rangle=l(l+1) \delta_{l l^{\prime}} \delta_{m m^{\prime}}$, it is easy to see that $\psi \in H^{1}$ if and only if $\operatorname{grad} \psi$ and $\operatorname{Grad} \psi$ both exist in $L^{2}$, and that there is an equivalence of norms

$$
\|\cdot\|_{H^{1}}^{2} \simeq\|\cdot\|^{2}+\|\operatorname{grad} \cdot\|^{2}+\|\operatorname{Grad} \cdot\|^{2} .
$$

Moreover, for $\psi \in H^{1}, D_{\text {hor }} \psi=u \cdot \operatorname{grad} \psi$.

The following Lemma gives some regularity properties of the operators introduced above and their commutators. These could be obtained easily using the formalism of Pseudo-Differential Operators. However, for the special cases considered here, they can also be proved directly.

Lemma 4.14. For each $s \in \mathbb{R}$, the operators

- $\operatorname{grad}_{i}, \operatorname{Grad}_{i}, D_{\text {hor }}: H^{s} \rightarrow H^{s-1}$ and their adjoints,

- $\left[\Lambda^{-1}, u_{i}\right]: H^{s} \rightarrow H^{s+2}$,

- and $\left[\Lambda^{-1}, \operatorname{grad}_{i}\right],\left[\Lambda^{-1}, \operatorname{Grad}_{i}\right]: H^{s} \rightarrow H^{s+1}$

are bounded. Here, $[A, B]:=A B-B A$ denotes the commutator.

Proof. The first statement is obvious from the definitions. For the second one,

$$
\left[\Lambda^{-1}, u_{i}\right] \psi=\sum_{k} \sum_{l m} \sum_{l^{\prime} m^{\prime}}\left(\lambda_{k l}^{-1}-\lambda_{k l^{\prime}}^{-1}\right)\left\langle\psi, \eta_{k l^{\prime} m^{\prime}}\right\rangle\left\langle u_{i} Y_{l^{\prime} m^{\prime}}, Y_{l m}\right\rangle \eta_{k l m}
$$

Since $u_{i}$ can be written as a linear combination of SHs of degree $1,\left\langle u_{i} Y_{l m^{\prime}}, Y_{l^{\prime} m^{\prime}}\right\rangle$ vanishes if $\left|l-l^{\prime}\right|>1$. The non-vanishing terms in the sum have the asymptotic behaviour

$$
\left|\lambda_{k l}^{-1}-\lambda_{k l^{\prime}}^{-1}\right|=\mathcal{O}\left(l \lambda_{k l}^{-3}\right) \quad \text { for }\left|l-l^{\prime}\right| \leq 1 \text {, as } k, l \rightarrow \infty .
$$

Together with the estimate $\left|\left\langle u_{i} Y_{l m^{\prime}}, Y_{l^{\prime} m^{\prime}}\right\rangle\right| \leq 1$, the assertion follows. Similarly,

$$
\left[\Lambda^{-1}, \operatorname{Grad}_{i}\right] \psi=\sum_{k} \sum_{l m} \sum_{l^{\prime} m^{\prime}}\left(\lambda_{k l}^{-1}-\lambda_{k l^{\prime}}^{-1}\right)\left\langle\psi, \eta_{k l^{\prime} m^{\prime}}\right\rangle\left\langle\operatorname{Grad}_{i} Y_{l^{\prime} m^{\prime}}, Y_{l m}\right\rangle \eta_{k l m}
$$

Again, $\left\langle\operatorname{Grad}_{i} Y_{l^{\prime} m^{\prime}}, Y_{l m}\right\rangle$ vanishes for $\left|l-l^{\prime}\right|>1$ and behaves like $\mathcal{O}(l)$ for $l \rightarrow \infty$, as can be seen from

$$
\begin{aligned}
\sum_{i}\left|\left\langle\operatorname{Grad}_{i} Y_{l^{\prime} m^{\prime}}, Y_{l m}\right\rangle\right|^{2} & \leq \sum_{i}\left\|\operatorname{Grad}_{i} Y_{l^{\prime} m^{\prime}}\right\|^{2}\left\|Y_{l m}\right\|^{2}=\sum_{i}\left\|\operatorname{Grad}_{i} Y_{l^{\prime} m^{\prime}}\right\|^{2} \\
& =\left\|\operatorname{Grad} Y_{l^{\prime} m^{\prime}}\right\|^{2}=l^{\prime}\left(l^{\prime}+1\right) .
\end{aligned}
$$

Together with $\left[\Lambda^{-1}\right.$, grad $]=0$, this shows the last assertion. 
We can now show the main theorem of this section, the proof of which uses techniques related to the proof of Hörmander's theorem on hypo-elliptic operators (cf. [Hör67]) found in [NHo5].

Theorem 4.15. $H_{\text {hor }}$ is a continuously embedded subspace of $H^{1 / 2}$.

Proof. Assume first that $\psi \in C_{\text {per }}^{\infty}$. Let $D_{1}:=\left[\mathrm{Grad}, D_{\text {hor }}\right]$. Then, $\operatorname{since} \operatorname{Grad}\left(a^{T} \cdot\right)(u)=$ $\left(1-u u^{T}\right) a$ for $a \in \mathbb{R}^{3}$, we have

$$
D_{1} \psi=\sum_{i=1}^{3}\left(\operatorname{Grad} u_{i}\right) \operatorname{grad}_{i} \psi=\left(1-u u^{T}\right) \operatorname{grad} \psi
$$

This implies

$$
\left\|D_{\text {hor }} \psi\right\|^{2}+\left\|D_{1} \psi\right\|^{2}=\|\operatorname{grad} \psi\|^{2}
$$

and hence

$$
\Lambda^{2}=1+D_{0}^{*} D_{0}+D_{1}^{*} D_{1}
$$

Thus

$$
\begin{aligned}
& \left\|\Lambda^{1 / 2} \psi\right\|^{2}=\left\langle\psi, \Lambda^{-1} \Lambda^{2} \psi\right\rangle=\left\|\Lambda^{-1 / 2} \psi\right\|^{2}+\sum_{i=0}^{1}\left\langle\psi, \Lambda^{-1} D_{i}^{*} D_{i} \psi\right\rangle \\
= & \left\|\Lambda^{-1 / 2} \psi\right\|^{2}+\sum_{i=0}^{1}\left\langle D_{i} \psi, \Lambda^{-1} D_{i} \psi\right\rangle+\left\langle\psi,\left[\Lambda^{-1}, D_{i}^{*}\right] \Lambda^{1 / 2} \Lambda^{-1 / 2} D_{i} \psi\right\rangle \\
\leq & \|\psi\|^{2}+\sum_{i=0}^{1}\left\|\Lambda^{-1 / 2} D_{i} \psi\right\|^{2}+c\|\psi\|\left\|\Lambda^{-1 / 2} D_{i} \psi\right\| \\
\leq & c\left(\|\psi\|+\left\|D_{0} \psi\right\|+\left\|\Lambda^{-1 / 2} D_{1} \psi\right\|\right)^{2},
\end{aligned}
$$

where all generic constants are denoted by $c$ for simplicity. We used that $\Lambda^{-1 / 2}$ and $\left[\Lambda^{-1}, D_{i}^{*}\right] \Lambda^{1 / 2}(i=1,2)$ are bounded. The latter follows using the regularity properties in Lemma 4.14, equation (4.15), and the Leibniz rule for commutators, i.e. $[A, B C]=$ $[A, B] C+B[A, C]$.

For the $D_{1}$-term above, we have

$$
\begin{aligned}
& \left\|\Lambda^{-1 / 2} D_{1} \psi\right\|^{2}=\sum_{i=1}^{3}\left\langle\Lambda^{-1} D_{1, i} \psi, D_{1, i} \psi\right\rangle \\
\leq & \sum_{i=1}^{3}\left|\left\langle\Lambda^{-1} D_{1, i} \psi, D_{\text {hor }} \operatorname{Grad}_{i} \psi\right\rangle\right|+\left|\left\langle\Lambda^{-1} D_{1, i} \psi, \operatorname{Grad}_{i} D_{\text {hor }} \psi\right\rangle\right| .
\end{aligned}
$$


The first term of this can be estimated by

$$
\begin{aligned}
& \left|\left\langle\Lambda^{-1} D_{1, i} \psi, D_{\text {hor }} \operatorname{Grad}_{i} \psi\right\rangle\right| \\
\leq & \left|\left\langle\Lambda^{-1} D_{1, i} D_{\text {hor }}^{*} \psi, \operatorname{Grad}_{i} \psi\right\rangle\right|+\left|\left\langle\left[D_{\text {hor }}^{*}, \Lambda^{-1} D_{1, i}\right] \psi, \operatorname{Grad}_{i} \psi\right\rangle\right| \\
\leq & c\left(\left\|D_{\text {hor }}^{*} \psi\right\|\left\|\operatorname{Grad}_{i} \psi\right\|+\|\psi\|\left\|\operatorname{Grad}_{i} \psi\right\|\right) \\
\leq & c\left(\|\psi\|+\left\|D_{0} \psi\right\|\right)^{2},
\end{aligned}
$$

where where $D_{\text {hor }}^{*}=-D_{\text {hor }}$ and the boundedness of $\Lambda^{-1} D_{1, i}$ and $\left[D_{\text {hor }}^{*} \Lambda^{-1} D_{1, i}\right]$ was used. Similarly,

$$
\begin{aligned}
& \left|\left\langle\Lambda^{-1} D_{1, i} \psi, \operatorname{Grad}_{i} D_{\text {hor }} \psi\right\rangle\right| \\
\leq & \left|\left\langle\Lambda^{-1} D_{1, i} \operatorname{Grad}_{i}^{*} \psi, D_{\text {hor }} \psi\right\rangle\right|+\left|\left\langle\left[\operatorname{Grad}_{i}^{*}, \Lambda^{-1} D_{1, i}\right] \psi, D_{\text {hor }} \psi\right\rangle\right| \\
\leq & c\left(\left\|\operatorname{Grad}_{i}^{*} \psi\right\|\left\|D_{\text {hor }} \psi\right\|+\|\psi\|\left\|D_{\text {hor }} \psi\right\|\right),
\end{aligned}
$$

since $\left[\operatorname{Grad}_{i}^{*}, \Lambda^{-1} D_{1, i}\right]$ is bounded. Using (4.15), the commutator between $\operatorname{Grad}_{i}$ and its adjoint is

$$
\left[\operatorname{Grad}_{i}, \operatorname{Grad}_{i}^{*}\right] \psi=2\left(1-u_{i}^{2}\right) \psi
$$

Hence,

$$
\left|\left\|\operatorname{Grad}_{i}^{*} \psi\right\|^{2}-\left\|\operatorname{Grad}_{i} \psi\right\|^{2}\right|=\left|\left\langle\psi,\left[\operatorname{Grad}_{i}^{*}, \operatorname{Grad}_{i}\right] \psi\right\rangle\right| \leq 2\|\psi\|^{2},
$$

so the second term can be estimated further by

$$
\left|\left\langle\Lambda^{-1} D_{1, i} \psi, \operatorname{Grad}_{i} D_{\text {hor }} \psi\right\rangle\right| \leq c\left(\|\psi\|+\left\|D_{0} \psi\right\|\right)^{2} .
$$

Putting everything together, we obtain

$$
\|\psi\|_{H^{1 / 2}}=\left\|\Lambda^{1 / 2} \psi\right\| \leq c\|\psi\|_{\text {hor }}
$$

for $\psi \in C_{\text {per }}^{\infty}$. Finally, Lemma 4.11 implies that the estimate also holds for arbitrary $\psi \in H_{\text {hor }}$.

Corollary 4.16. $H_{\text {hor }}$ is compactly embedded in $L^{2}$.

Proof. This follows immediately from the fact that $H^{s}$ is compactly embedded in $L^{2}$ for any $s>0$.

\subsection{Convergence Result}

We conclude the theoretical analysis of our method by summarizing the results in a convergence theorem for ODF reconstruction with $H_{\text {hor }}$-penalty and linear forward operator $T$, for example the spherical convolution operator from (2.17). The main difficulty in applying Theorem 3.12 in this setting is the convergence of the discretized forward operator, since it is not smoothing in the spatial variable, and therefore compactness properties are not clear. This is solved by the results of the preceding section. 
Theorem 4.17. Let $Y$ be a Hilbert space and $T: L^{2} \rightarrow Y$ a bounded linear operator. Denote by $\iota: H_{\text {hor }} \rightarrow L^{2}$ the embedding and let $T_{h}:=Q_{h} T \iota P_{h}$ for orthogonal projections $P_{h}$ in $H_{\text {hor }}$ and $Q_{h}$ in $Y$ fulfilling $P_{h} \rightarrow \mathbb{1}$ pointwise on $\mathcal{R}\left((T \iota)^{*}\right)$ and $Q_{h} \rightarrow \mathbb{1}$ pointwise on $\mathcal{R}(T \iota)$ as $h \rightarrow 0$. Moreover, let $\mathrm{C} \subset \mathrm{H}_{\mathrm{hor}}$ be a closed and convex constraint set.

Then, given noisy data $y^{\delta} \in Y$ with $\left\|T \psi^{\dagger}-y^{\delta}\right\|_{Y} \leq \delta$, the discrete, Tikhonov-regularized solution

$$
\psi_{\alpha, h}^{\delta}=\underset{\psi \in C}{\operatorname{argmin}}\left(\left\|T_{h} \psi-y^{\delta}\right\|_{Y}^{2}+\alpha\|\psi\|_{\text {hor }}^{2}\right)
$$

fulfills

$$
\left\|\psi_{\alpha(\delta, h), h}^{\delta}-\psi^{\dagger}\right\|_{\text {hor }} \rightarrow 0 \quad \text { as } \delta, h \rightarrow 0
$$

for a parameter choice rule $\alpha(\delta, h)$ satisfying

$$
\alpha(\delta, h) \rightarrow 0, \quad \frac{\delta^{2}}{\alpha(\delta, h)} \rightarrow 0 \quad \text { and } \quad \frac{\left\|T \iota-T_{h}\right\|^{2}}{\alpha(\delta, h)}<c \quad \text { as } \delta, h \rightarrow 0
$$

with some $c>0$.

Proof. Corollary 4.16 and Lemma 3.6 show that $\left\|T \iota-T_{h}\right\| \rightarrow 0$. As discussed in Remark 3.13, the parameter choice rule fulfills the conditions of the first part of Theorem 3.12 for $X=H_{\text {hor }}$ and functionals $\mathcal{T}, S$ and $\mathcal{R}$ defined by (3.15). Therefore, the assertion follows.

Moreover, as in Theorem 3.12, if $\psi^{\dagger}$ fulfills a variational smoothness assumption, then for suitable parameter choice rules, convergence rates in terms of the data noise $\delta$ and the discretization error $\left\|T \iota-T_{h}\right\|$ can be obtained. Note however that Lemma 3.6 does not give an explicit estimate for $\left\|T \iota-T_{h}\right\|$. 



\section{Discretization and Implementation}

\subsection{Discretization of the spherical convolution operator}

We aim to discretize the spherical convolution operator from (2.17),

$$
T: H_{\text {hor }} \rightarrow L^{2}, \quad T=\left(\mathbb{1}_{L^{2}(\Omega)} \otimes T_{0}\right) \circ l,
$$

where $\iota: H_{\text {hor }}\left(\Omega \times S^{2}\right) \rightarrow L^{2}\left(\Omega \times S^{2}\right) \simeq L^{2}(\Omega) \otimes L^{2}\left(S^{2}\right)$ is the compact embedding and $T_{0}: L^{2}\left(S^{2}\right) \rightarrow L^{2}\left(S^{2}\right)$ is defined by

$$
T_{0} \psi(q)=\int_{S^{2}} K(q \cdot u) \psi(u) d u
$$

$\Omega$ is again the cube

$$
\Omega:=\prod_{i=1}^{3}\left(-\frac{L_{i}}{2}, \frac{L_{i}}{2}\right) .
$$

Discretization is performed by choosing basis functions $\left(\lambda_{i}\right)_{i=1}^{N_{x}} \subset L^{2}(\Omega),\left(\varphi_{j}\right)_{j=1}^{N_{u}} \subset$ $L^{2}\left(S^{2}\right)$ and $\left(\eta_{k}\right)_{k=1}^{N_{q}} \subset L^{2}\left(S^{2}\right)$ such that $\lambda_{i} \otimes \varphi_{j} \in H_{\text {hor }}$ for all $i, j$. $P_{\lambda, \varphi}$ denotes the orthogonal projection onto the discrete space

$$
X_{\lambda, \varphi}:=\operatorname{span}\left\{\lambda_{i} \otimes \varphi_{j}: i=1 \ldots N_{x}, j=1 \ldots N_{u}\right\} \subset H_{\text {hor }}
$$

and $Q_{\lambda, \eta}$ is the projection onto

$$
Y_{\lambda, \eta}:=\operatorname{span}\left\{\lambda_{i} \otimes \eta_{j}: i=1 \ldots N_{x}, j=1 \ldots N_{q}\right\} \subset L^{2} .
$$

The discretized operator $T_{\lambda, \varphi, \eta}:=Q_{\lambda, \eta} T P_{\lambda, \varphi}$ is then expanded in this basis as

$$
T_{\lambda, \varphi, \eta}\left(\lambda_{i} \otimes \varphi_{j}\right)=\sum_{k} B_{k j} \lambda_{i} \otimes \eta_{k}
$$

with $B \in \mathbb{R}^{N_{q} \times N_{u}}$, i.e. the discretization does not depend on the choice of spatial basis $\left(\lambda_{i}\right)$. For notational simplicity, we will not indicate the dependence of $B$ on the basis functions. Moreover, if no confusion arises, we will sometimes identify $B$ with $\mathbb{1} \otimes B$, which means that applying $B$ to $c \in \mathbb{R}^{N_{x} \times N_{u}}$ is understood as

$$
(B c)_{i k}:=\sum_{j} B_{k j} c_{i j}
$$


The projected ODF and noisy data are expanded in the discrete basis as

$$
P_{\lambda, \varphi} \psi=\sum_{i, j} c_{i j} \lambda_{i} \otimes \varphi_{j}
$$

and

$$
Q_{\lambda, \eta} y^{\delta}=\sum_{i, k} z_{i k}^{\delta} \lambda_{i} \otimes \eta_{k}
$$

Moreover, the Gramian matrices

$$
G_{i j, i^{\prime} j^{\prime}}^{X}=\left\langle\lambda_{i} \otimes \varphi_{j}, \lambda_{j^{\prime}} \otimes \varphi_{j^{\prime}}\right\rangle_{\text {hor }}
$$

and similarly for $G^{Y}$, are needed. With this notation, the discrete problem we want to solve can be written as

$$
\underset{c \in C}{\operatorname{argmin}}\left(\left\|\sqrt{G^{Y}}\left(B c-z^{\delta}\right)\right\|^{2}+\alpha\left\|\sqrt{G^{X}} c\right\|^{2}\right),
$$

where $C$ is a suitable approximation of the constraint set

$$
\left\{c \in \mathbb{R}^{N_{x} \times N_{u}}: \sum_{i j} c_{i j} \lambda_{i} \otimes \varphi_{j} \geq 0\right\} .
$$

\subsubsection{Trigonometric basis in $\Omega$}

As the MRI forward operator is essentially the Fourier transform, a natural choice for the spatial discretization is the space spanned by a subset of the trigonometric polynomials (4.13),

$$
\xi_{k}(x)=|\Omega|^{-\frac{1}{2}} \prod_{j=1}^{3} \exp \left(2 \pi i L_{j}^{-1} k_{j} x_{j}\right),
$$

where $k \in \mathbb{Z}^{3}$. They form an orthogonal basis in $H^{s}(\Omega)$ for each $s \in \mathbb{R}$, and fulfill $\left\|\xi_{k}\right\|_{H^{s}}=\left(1+\|k\|^{2}\right)^{s / 2}$. Discretization is performed by restricting to $\xi_{k}$ for $k \in \mathcal{K}:=\{k \in$ $\left.\mathbb{Z}^{3}:\left|k_{i}\right| \leq K_{i}, i=1,2,3\right\}$ for some $K \in \mathbb{Z}^{3}$. The discrete constraint set

$$
C_{\mathcal{K}}^{\xi}=\left\{\hat{g} \in \mathbb{R}^{|\mathcal{K}|}: \sum_{k \in \mathcal{K}} \hat{g}_{k} \xi_{k} \geq 0\right\}
$$

is difficult to implement in practice. We circumvent this problem by only checking the constraint on the finite grid $\left(x_{p}\right)_{p \in K} \subset \mathbb{R}^{3}$, where $\left(x_{p}\right)_{j}=\left(2 K_{j}+1\right)^{-1} L_{j} p_{j}$, i.e.

$$
\tilde{C}_{\mathcal{K}}^{\xi}=\left\{\hat{g} \in \mathbb{R}^{|\mathcal{K}|}: \forall p \in \mathcal{K}: \sum_{k \in \mathcal{K}} \hat{g}_{k} \xi_{k}\left(x_{p}\right) \geq 0\right\} .
$$

Alternatively, the Lagrange basis

$$
\lambda_{p}(x)=\frac{|\Omega|^{1 / 2}}{|\mathcal{K}|} \sum_{k \in \mathcal{K}} \xi_{k}\left(x-x_{p}\right)
$$


with $p \in \mathcal{K}$ can be used. This basis has the property that $\lambda_{p}\left(x_{p^{\prime}}\right)=\delta_{p p^{\prime}}$, and hence the approximated constraint set in this basis simply becomes

$$
\tilde{C}_{\mathcal{K}}^{\lambda}=\left\{g \in \mathbb{R}^{|\mathcal{K}|}: g \geq 0\right\}
$$

It should be noted that the convergence result in Theorem 3.12 does not cover approximating the constraint sets in this way.

\subsection{Linear basis function on $S^{2}$}

While the choice of spatial basis functions can be seen as natural, there are different possibilities for the basis functions on $S^{2}$. We will discuss two possible choices.

One possibility is a simple linear basis with respect to a suitable triangulation. This choice greatly simplifies the constraint, but leads to a voxel-wise non-sparse forward operator. It is, however, still sparse on the whole space.

To define the basis functions $\varphi_{u} \in L^{2}\left(S^{2}\right)$, we start by choosing a suitable finite set of points $\sigma \subset S^{2}$, and determine a triangulation $\mathcal{T}_{\sigma}$ of the boundary of the convex hull of $\sigma$. So each $T \in \mathcal{T}_{\sigma}$ is an affine image of the standard simplex $\left\{x \in \mathbb{R}^{3}: x \geq 0, \sum_{i} x_{i}=1\right\}$, with each vertex of $T$ being a point in $\sigma$. Additionally, two different elements of $\mathcal{T}_{\sigma}$ are either disjoint or intersect along a shared edge or a single shared vertex, and

$$
\cup \mathcal{T}_{\sigma}:=\cup_{T \in \mathcal{T}_{\sigma}} T=\partial(\text { convhull } \sigma),
$$

where $\partial$ denotes the boundary. Such a triangulation can be obtained for example using MATLAB's convhulln function. We will assume that $\sigma$ is such that the map

$$
\gamma_{\sigma}: \cup \mathcal{T}_{\sigma} \rightarrow S^{2}, \quad p \mapsto \hat{p}:=\frac{p}{\|p\|}
$$

is a homeomorphism. For each $u \in \sigma$, let $\tilde{\varphi}_{u}: \cup \mathcal{T}_{\sigma} \rightarrow \mathbb{R}$ be the function that is linear on the faces of $\mathcal{T}_{\sigma}$ and fulfills $\tilde{\varphi}_{u}(v)=\delta_{u v}$ for all $v \in \sigma$. Then the basis function $\varphi_{u}$ is defined by

$$
\varphi_{u}=\tilde{\varphi}_{u} \circ \gamma_{\sigma}^{-1}
$$

Regarding the constraint, note that $\sum_{u \in \sigma} f_{u} \varphi_{u} \geq 0$ if and only if $f \geq 0$.

The point set $\sigma \subset S^{2}$ should be as uniform as possible. We follow the approach from [Frao8] using platonic solids, starting with an icosahedron and subdividing each face into $n^{2}$ identical triangles corresponding to the vertices

$$
\left\{v \in \mathbb{R}^{3}: n v \in \mathbb{Z}^{3}, v \geq 0, \sum_{i} v_{i}=1\right\}
$$

on the standard simplex. The resulting points are then radially projected onto $S^{2}$, resulting in point sets with $10 n^{2}+2$ points. 


\subsubsection{Approximation properties}

We want to show that the orthogonal projection $P_{\mathcal{K}, \sigma}$ onto $\operatorname{span}\left\{\xi_{k} \otimes \varphi_{u}\right\}_{k \in \mathcal{K}, u \in \sigma} \subset H_{\text {hor }}$ fulfills $P_{\mathcal{K}, \sigma} \psi \rightarrow \psi$ for all $\psi \in H_{\text {hor }}$ as the discretization $(\mathcal{K}, \sigma)$ is refined in a suitable way. To investigate the approximation properties, we first need a result on approximation on the triangulation $\cup \mathcal{T}_{\sigma}$.

Lemma 5.1. For each triangle $T \in \mathcal{T}_{\sigma}$, let $h_{T}$ be the radius of the circumscribed circle and $\rho_{T}$ the radius of the inscribed circle. Assume that

$$
\sup _{T \in \mathcal{T}_{\sigma}} \frac{h_{T}}{\rho_{T}} \leq \kappa_{\sigma}
$$

for some $\kappa_{\sigma}>0$, and let $h_{\sigma}:=\sup _{T \in \mathcal{T}_{\sigma}} h_{T}$ be sufficiently small. For functions $f: \cup \mathcal{T}_{\sigma} \rightarrow \mathbb{R}$ satisfying $\left.f\right|_{T} \in H^{2}(T)$ for all $T \in \mathcal{T}_{\sigma}$, define the interpolation operator $I_{\sigma}$ by

$$
I_{\sigma}(f):=\sum_{u \in \sigma} f(u) \tilde{\varphi}_{u}
$$

Then

$$
\left\|f-I_{\sigma} f\right\|_{H^{1}(T)} \leq C h_{\sigma}\|f\|_{H^{2}(T)}
$$

for all $T \in \mathcal{T}_{\sigma}$. The constant $C$ depends on $\sigma$ only through dependence on $\kappa_{\sigma}$.

Proof. See [BSo2, Theorem 4.4.20].

This can be translated this into an estimate on $S^{2}$ using transformation properties of Sobolev norms. In general, let $\eta: T \rightarrow S$ be a diffeomorphism between manifolds $T$ and $S$, and $f \in H^{2}(S)$. Then due to the chain rule and transformation formula, the Sobolev semi-norms on $S$ and $T$ are related by

$$
|f \circ \eta|_{H^{1}(T)}^{2} \leq C\left\|\operatorname{det} D \eta^{-1}\right\|_{\infty}\|\eta\|_{C^{1}}^{2}|f|_{H^{1}(S)}^{2}
$$

and

$$
|f \circ \eta|_{H^{2}(T)}^{2} \leq C\left\|\operatorname{det} D \eta^{-1}\right\|_{\infty}\left(\|\eta\|_{C^{1}}^{4}|f|_{H^{2}(S)}^{2}+\|\eta\|_{C^{2}}^{2}|f|_{H^{1}(S)}^{2}\right),
$$

for some constant $C>0$ depending only on the dimension of $S$ and $T$.

Now let $T \in \mathcal{T}_{\sigma}$ be a triangle with unit normal vector $n_{T} \in \mathbb{R}^{3}$ oriented to point away from the origin, i.e. such that $d_{T}:=n_{T} \cdot p>0$ for all $p \in T$. Note that $d_{T}$ is a constant, namely the distance of $T$ from the origin. Then $\left.\gamma_{\sigma}\right|_{T}: T \rightarrow \gamma_{\sigma}(T)$, is invertible with $\left.\gamma_{\sigma}\right|_{T} ^{-1}(u)=d_{T}\left(n_{T} \cdot u\right)^{-1} u$. The derivatives are

$$
\left.D \gamma_{\sigma}\right|_{T}(p)=\frac{1}{\|p\|}\left(\mathbb{1}-\hat{p} \hat{p}^{T}\right) \quad \text { and }\left.\quad D \gamma_{\sigma}\right|_{T} ^{-1}(u)=\frac{d_{T}}{n_{T} \cdot u}\left(\mathbb{1}-\frac{u n_{T}^{T}}{n_{T} \cdot u}\right)
$$


Direct calculation yields

$$
\left\|\left.D \gamma_{\sigma}\right|_{T}(p)\right\|_{2}=\frac{1}{\|p\|} \leq \frac{1}{d_{T}}, \quad\left\|\left.D \gamma_{\sigma}\right|_{T} ^{-1}(u)\right\|_{2}=\frac{d_{T}}{\left(n_{T} \cdot u\right)^{2}} \leq \frac{1}{d_{T}}
$$

and

$$
\left.\operatorname{det} D \gamma_{\sigma}\right|_{T} ^{-1}(u)=\frac{d_{T}^{2}}{\left(n_{T} \cdot u\right)^{3}} \leq \frac{1}{d_{T}} .
$$

Moreover, for the second derivative

$$
\left(\left.D^{2} \gamma_{\sigma}\right|_{T}(p) v\right)_{i j}=\frac{1}{\|p\|^{2}}\left((\hat{p} \cdot v)\left(3 \hat{p}_{i} \hat{p}_{j}-\delta_{i j}\right)-\hat{p}_{i} v_{j}-v_{i} \hat{p}_{j}\right)
$$

we obtain

$$
\left\|\left.D^{2} \gamma_{\sigma}\right|_{T}(p)\right\|_{2}=\frac{\sqrt{2}}{\|p\|^{2}} \leq \frac{\sqrt{2}}{d_{T}^{2}} .
$$

So as long as $d_{T}$ is bounded away from 0 - as should be the case for any reasonable approximation of $S^{2}-$, the estimates (5.8) yield

$$
\left\|\left.g \circ \gamma_{\sigma}\right|_{T} ^{-1}\right\|_{H^{1}\left(\gamma_{\sigma}(T)\right)} \leq C\|g\|_{H^{1}(T)}
$$

for $g \in H^{1}(T)$ and

$$
\left\|\left.f \circ \gamma_{\sigma}\right|_{T}\right\|_{H^{2}(T)} \leq C\|f\|_{H^{2}\left(\gamma_{\sigma}(T)\right)}
$$

for $f \in H^{2}\left(\gamma_{\sigma}(T)\right)$ and some generic constant $C$. Hence, using (5.7), we obtain for $f \in H^{2}\left(S^{2}\right)$

$$
\begin{aligned}
\left\|f-I_{\sigma}\left(f \circ \gamma_{\sigma}\right) \circ \gamma_{\sigma}^{-1}\right\|_{H^{1}\left(S^{2}\right)}^{2} & =\sum_{T \in \mathcal{T}_{\sigma}}\left\|\left.f\right|_{\gamma_{\sigma}(T)}-\left.I_{\sigma}\left(f \circ \gamma_{\sigma}\right) \circ \gamma_{\sigma}\right|_{T} ^{-1}\right\|_{H^{1}\left(\gamma_{\sigma}(T)\right)}^{2} \\
& \leq C \sum_{T \in \mathcal{T}_{\sigma}}\left\|\left.f \circ \gamma_{\sigma}\right|_{T}-\left.I_{\sigma}\left(f \circ \gamma_{\sigma}\right)\right|_{T}\right\|_{H^{1}(T)}^{2} \\
& \leq C h_{\sigma}^{2} \sum_{T \in \mathcal{T}_{\sigma}}\left\|\left.f \circ \gamma_{\sigma}\right|_{T}\right\|_{H^{2}(T)}^{2} \\
& =C h_{\sigma}^{2}\|f\|_{H^{2}\left(S^{2}\right)^{\prime}}^{2}
\end{aligned}
$$

with $C$ depending only on $\kappa_{\sigma}$ as long as $\inf _{T \in \mathcal{T}_{\sigma}} d_{T} \geq d_{0}>0$. Using this estimate, pointwise convergence of the discretization projection in $H_{\text {hor }}$ can be proved:

Lemma 5.2. For $n \in \mathbb{N}$, let $\sigma_{n} \subset S^{2}$ be such that the assumptions of Lemma 5.1 hold for each $n$ with $\kappa_{\sigma_{n}}=\kappa$ independent of $n$ and $h_{\sigma_{n}} \rightarrow 0$ as $n \rightarrow \infty$, and such that $\inf _{T \in \mathcal{T}_{\sigma_{n}}} d_{T} \geq d_{0}$ for some $d_{0}>0$. Moreover, let $\mathcal{K}_{n}=\left\{k \in \mathbb{Z}^{3}:\left|k_{i}\right| \leq K_{i}^{n}, i=1,2,3\right\}$ with $K_{i}^{n} \rightarrow \infty$ as $n \rightarrow \infty$ for $i=1,2,3$. Then $P_{\mathcal{K}_{n}, \sigma_{n}} \rightarrow 1$ pointwise in $H_{\text {hor }}$. 


\section{Discretization and Implementation}

Proof. Let $s \geq 1$ and $X^{s}:=H^{1}(\Omega) \otimes H^{s}\left(S^{2}\right) \subset H_{\text {hor }}$. Then, by definition of the tensor product, since $\left\{\xi_{k}\right\}_{k \in \mathbb{Z}^{3}}$ form a basis in $H^{1}(\Omega), \psi \in X^{s}$ can be written as

$$
\psi=\sum_{k \in \mathbb{Z}^{3}} \xi_{k} \otimes a_{k}
$$

with a sequence $a_{k} \in H^{s}\left(S^{2}\right)$ fulfilling

$$
\|\psi\|_{X^{s}}^{2}=\sum_{k \in \mathbb{Z}^{3}}\left(1+\|k\|^{2}\right)\left\|a_{k}\right\|_{H^{s}\left(S^{2}\right)}^{2}<\infty .
$$

Moreover, $\|\psi\|_{\text {hor }} \leq\|\psi\|_{H^{1}} \leq\|\psi\|_{X^{1}}$. Let $\psi \in X^{2}$. Then, since $X_{\mathcal{K}_{n}, \sigma_{n}} \subset X^{1}$,

$$
\begin{aligned}
& \left\|P_{\mathcal{K}_{n}, \sigma_{n}} \psi-\psi\right\|_{\text {hor }}^{2}=\inf _{\eta \in X_{\mathcal{K}_{n}, \sigma_{n}}}\|\eta-\psi\|_{\text {hor }}^{2} \leq \inf _{\eta \in X_{\mathcal{K}_{n}, \sigma_{n}}}\|\eta-\psi\|_{X^{1}}^{2} \\
= & \inf \left\{\left\|\sum_{k \in \mathcal{K}_{n}} \sum_{u \in \sigma_{n}} b_{k u} \xi_{k} \otimes \varphi_{u}-\psi\right\|_{X^{1}}^{2}: b \in \mathbb{R}^{\left|\mathcal{K}_{n}\right| \times\left|\sigma_{n}\right|}\right\} .
\end{aligned}
$$

Inserting the expansion of $\psi$, the norm here can be rewritten:

$$
=\inf \left\{\left\|\sum_{k \in \mathcal{K}_{n}} \xi_{k} \otimes\left(\sum_{u \in \sigma_{n}} b_{k u} \varphi_{u}-a_{k}\right)+\sum_{k \in \mathbb{Z}^{3} \backslash \mathcal{K}_{n}} \xi_{k} \otimes a_{k}\right\|_{X^{1}}^{2}: b \in \mathbb{R}^{\left|\mathcal{K}_{n}\right| \times\left|\sigma_{n}\right|}\right\},
$$

so the infimum simplifies to

$$
\begin{aligned}
& =\sum_{k \in \mathcal{K}_{n}}\left(1+\|k\|^{2}\right) \inf _{b \in \mathbb{R}^{\mid \sigma_{n}} \mid}\left\|\sum_{u \in \sigma_{n}} b_{u} \varphi_{u}-a_{k}\right\|_{H^{1}\left(S^{2}\right)}^{2}+\sum_{k \in \mathbb{Z}^{3} \backslash \mathcal{K}_{n}}\left(1+\|k\|^{2}\right)\left\|a_{k}\right\|_{H^{1}\left(S^{2}\right)}^{2} \\
& \leq C h_{\sigma_{n}} \sum_{k \in \mathcal{K}_{n}}\left(1+\|k\|^{2}\right)\left\|a_{k}\right\|_{H^{2}\left(S^{2}\right)}^{2}+\sum_{k \in \mathbb{Z}^{3} \backslash \mathcal{K}_{n}}\left(1+\|k\|^{2}\right)\left\|a_{k}\right\|_{H^{1}\left(S^{2}\right)}^{2}
\end{aligned}
$$

In the last line, (5.9) was used. The assumptions now show that $P_{\mathcal{K}_{n}, \sigma_{n}} \rightarrow 1$ pointwise on $X^{2}$ as $n \rightarrow \infty$. Since $C_{\text {per }}^{\infty} \subset X^{2}$, Lemma 4.11 implies that $X^{2}$ is dense in $H_{\text {hor }}$. Therefore, for all $\psi \in H_{\text {hor }}$, one can choose $\psi_{\varepsilon} \in X^{2}$ with $\left\|\psi-\psi_{\varepsilon}\right\| \leq \varepsilon$. Together with $\left\|P_{\mathcal{K}_{n}, \sigma_{n}}\right\|=1$, it follows that for $n$ large enough

$$
\left\|P_{\mathcal{K}_{n}, \sigma_{n}} \psi-\psi\right\|_{\text {hor }} \leq\left(1+\left\|P_{\mathcal{K}_{n}, \sigma_{n}}\right\|\right)\left\|\psi-\psi_{\varepsilon}\right\|_{\text {hor }}+\left\|P_{\mathcal{K}_{n}, \sigma_{n}} \psi_{\varepsilon}-\psi_{\varepsilon}\right\|_{\text {hor }} \leq 2 \varepsilon,
$$

so $P_{\mathcal{K}_{n}, \sigma_{n}} \rightarrow \mathbb{1}$ pointwise on all of $H_{\text {hor }}$.

\subsubsection{The Gramian matrix}

The Gramian matrix for the Laplace-Beltrami on $S^{2}$ operator can be computed using its weak definition and the transformation formula,

$$
\begin{aligned}
& \left\langle\varphi_{u}, \Delta \varphi_{v}\right\rangle=-\int_{S^{2}} \operatorname{Grad} \varphi_{u}(w)^{T} \operatorname{Grad} \varphi_{v}(w) d w \\
& =-\sum_{T \in \mathcal{T}_{\sigma}} \int_{T} \operatorname{Grad} \tilde{\varphi}_{u}(p)^{T} A_{T}(p) \operatorname{Grad} \tilde{\varphi}_{v}(p) d p
\end{aligned}
$$

60 
where

$$
A_{T}=\left(\left.\left.D \gamma_{\sigma}\right|_{T} ^{-1} \circ \gamma_{\sigma}\right|_{T}\right)\left(\left.\left.D \gamma_{\sigma}\right|_{T} ^{-1} \circ \gamma_{\sigma}\right|_{T}\right)^{T}\left|\operatorname{det} D \gamma_{\sigma}\right|_{T} \mid
$$

Using the explicit expressions above, this yields

$$
\left\langle\varphi_{u}, \Delta \varphi_{v}\right\rangle=-\sum_{T \in \mathcal{T}_{\sigma}} d_{T} \int_{T} \frac{1}{\|p\|} \operatorname{Grad} \tilde{\varphi}_{u}(p)^{T}\left(1+\frac{p p^{T}}{d_{T}^{2}}\right) \operatorname{Grad} \tilde{\varphi}_{v}(p) d p .
$$

which can be evaluated in a pre-computation step using e.g. Gauss-Legendre quadrature on the triangles $T \in \mathcal{T}_{\sigma}$. The Gramian matrix for the $L^{2}$-norm can be computed similarly using the transformation formula. The horizontal derivative can be computed most easily in the Fourier basis (5.3), for which one has to evaluate

$$
\int_{S^{2}}(k \cdot w)^{2} \varphi_{u}(w) \varphi_{v}(w) d w, \quad k \in \mathcal{K}
$$

When using the Fourier-Lagrange basis (5.4), Fast Fourier Transforms (FFTs) can be used to change to the Fourier basis and back. Note that this requires two FFTs for each point in $\sigma$. Putting all these together yields the Gramian matrix

$$
\left\langle\xi_{k} \otimes \varphi_{u}, \xi_{k^{\prime}} \otimes \varphi_{v}\right\rangle_{\mathrm{hor}}=\delta_{k k^{\prime}} G_{k, u v^{\prime}}^{X}
$$

with a sparse matrix $G_{k}^{X} \in R^{|\sigma| \times|\sigma|}$ for each $k \in \mathcal{K}$. If memory permits, these can be precomputed and stored as a single large sparse matrix. Otherwise, one can just precompute

$$
\int_{S^{2}} w_{i} w_{j} \varphi_{u}(w) \varphi_{v}(w) d w \quad i, j \in\{1,2,3\}
$$

and perform the contractions with $k_{i} k_{j}$ separately for each application of $G^{X}$.

\subsubsection{Basis in the codomain}

The basis $\left\{\eta_{j}\right\}$ in the codomain poses a conceptual problem, since the most natural way to model the DW measurement are point evaluations. From these, the coefficients $z^{\delta}$ of the projection $Q_{h} y^{\delta}$ have to be estimated, which can in general only be done approximately, possible enlarging the data noise bound $\delta$. Good estimates of $z^{\delta}$ can be expected when choosing a basis approximating delta distributions centered at the sampling points $\left\{q_{j}\right\}$, effectively modelling the measured data not as a point evaluation, but as an average over a sufficiently small region around the $q_{j}$. While choosing small regions is preferable to achieve a good approximation of the data, convergence of the corresponding projection $Q_{h}$ is slower for smaller regions, since more basis functions are needed to effectively cover the space.

For simplicity of implementation, we will use point evaluations despite the fact that the convergence Theorem 3.12 is not applicable to that case. This means that the coefficients 
$z^{\delta}$ are directly given by the measured data, while the convolution operator (5.1) is implemented as a matrix

$$
B_{j u}=\int_{S^{2}} K\left(q_{j} \cdot v\right) \varphi_{u}(v) d v
$$

in each voxel.

\subsection{The Semi-smooth Newton Method}

We now present the algorithm used to solve the constrained optimization problem. The method - the primal-dual active set method — was introduced in [BIK99] and analyzed in [HIKo3] by showing that it can be interpreted as a semi-smooth Newton method. This section follows the theoretical basis from that work, but will only describe the finite-dimensional case, circumventing some of the more intricate parts of the general setting.

The problem has the general form

$$
\underset{x \geq 0}{\operatorname{argmin}}\|T x-y\|^{2}
$$

where $T$ now contains both the forward operator and the Gramian matrix from the regularization penalty. Note in particular the simple form of the constraint due to the choice of basis above. There exist Lagrange multipliers $\lambda \in \mathbb{R}^{n}$ such that

$$
\begin{gathered}
T^{*} T x-\lambda=T^{*} y \\
x \geq 0, \lambda \geq 0, \lambda^{T} x=0 .
\end{gathered}
$$

Conditions (5.11a) can be rewritten as

$$
\lambda=\max (0, \lambda-c x)
$$

for any $c>0$. Here, all inequalities and the max-operation are interpreted componentwise. Thus (5.10) is equivalent to finding a zero of $S: \mathbb{R}^{2 n} \rightarrow \mathbb{R}^{2 n}$,

$$
S(x, \lambda):=\left(\begin{array}{c}
T^{*} T x-\lambda-T^{*} y \\
\lambda-\max (0, \lambda-c x)
\end{array}\right)
$$

A fast algorithm for finding zeros is Newton's method, which unfortunately is only applicable to differentiable functions. $S$ is not differentiable in the usual way, but turns out to be differentiable under a modified notion of differentiability, for which the Newton method is still applicable. 
Definition 5.3. Let $U \subset \mathbb{R}^{n}$ be an open set and $F: U \rightarrow \mathbb{R}^{m}$. Then $f: U \rightarrow \mathbb{R}^{m \times n}$ is called a Newton derivative of $F$ if, for all $x \in U$,

$$
\lim _{h \rightarrow 0} \frac{\|F(x+h)-F(x)-f[x+h] h\|}{\|h\|}=0 .
$$

In comparison to the definition of the Fréchet derivative, $F^{\prime}[x]$ is replaced by $f[x+h]$. In general, Newton derivatives are not unique.

We are particularly interested in a Newton derivative of the map $\mathbb{R}^{n} \ni x \mapsto \max (0, x)$. It is given by

$$
m[x]:=\operatorname{diag}\left(\theta\left(x_{1}\right), \ldots, \theta\left(x_{n}\right)\right),
$$

where $\theta(x)=0$ for $x \leq 0$ and $\theta(x)=1$ for $x>0$ is the Heaviside step function. ${ }^{1}$ Indeed, for $i=1, \ldots, n$, if $x_{i} \neq 0$ and $\left|h_{i}\right|<\left|x_{i}\right|$, then

$$
\left|\max \left(0, x_{i}+h_{i}\right)-\max \left(0, x_{i}\right)-\theta\left(x_{i}+h_{i}\right) h_{i}\right|=0 .
$$

On the other hand, if $x_{i}=0,\left|\max \left(0, h_{i}\right)-\theta\left(h_{i}\right) h_{i}\right|=0$ holds true for arbitrary $h_{i}$. So

$$
\frac{\|\max (0, x+h)-\max (0, h)-m[x+h] h\|}{\|h\|}=0
$$

if $\|h\| \neq 0$ is small enough.

The Semi-smooth Newton Method now is simply Newton's method with the derivatives replaced by a Newton derivative.

Theorem 5.4. Let $U \subset \mathbb{R}^{n}$ be open, $F: U \rightarrow \mathbb{R}^{m}$ and $x^{*} \in U$ be a zero of $F$. If $F$ has a Newton derivative $f$ for which $\left\|f[x]^{-1}\right\|$ is uniformly bounded for $x$ in a neighborhood of $x^{*}$, then the Newton iteration

$$
x_{k+1}=x_{k}-f\left[x_{k}\right]^{-1} F\left(x_{k}\right)
$$

converges superlinearly to $x^{*}$ provided $\left\|x_{0}-x^{*}\right\|$ is sufficiently small.

Proof. See [HIKo3, Theorem 1.1].

It is easy to see from the definition that if $f$ is a Newton derivative for $F$ and $A: x \mapsto$ $A^{\prime} x+a$ is an affine mapping, then $x \mapsto f[A x] A^{\prime}$ is Newton derivative for $F \circ A$, and $x \mapsto A^{\prime} f[x]$ is a Newton derivative for $A \circ F$. Hence, the function $S$ defined in (5.12) has a Newton derivative

$$
s[x, \lambda]=\left(\begin{array}{cc}
T^{*} T & -\mathbb{1} \\
c m[\lambda-c x] & \mathbb{1}-m[\lambda-c x]
\end{array}\right) .
$$

\footnotetext{
${ }^{1}$ Actually, the value of $\theta(0)$ does not matter; any value yields a Newton derivative.
} 


\section{Discretization and Implementation}

Introduce the active set

$$
\mathcal{A}:=\left\{i: \lambda_{i}-c x_{i}>0\right\},
$$

and its complement $\mathcal{I}:=\mathcal{A}^{c}$, the inactive set. Then

$$
P_{\mathcal{A}}=m[\lambda-c x]
$$

is the projection onto the active set, and $s$ can be written as

$$
s[x, \lambda]=\left(\begin{array}{ll}
T^{*} T & -\mathbb{1} \\
c P_{\mathcal{A}} & P_{\mathcal{I}}
\end{array}\right)
$$

If

$$
s[x, \lambda]\left(\begin{array}{c}
\delta_{x} \\
\delta_{\lambda}
\end{array}\right)=\left(\begin{array}{c}
v \\
w
\end{array}\right)
$$

then

$$
\begin{gathered}
\delta_{x}=c^{-1} P_{\mathcal{A}} w+P_{\mathcal{I}}\left(P_{\mathcal{I}} T^{*} T P_{\mathcal{I}}\right)^{\dagger} P_{\mathcal{I}}\left(v+w-c^{-1} T^{*} T P_{\mathcal{A}} w\right), \\
\delta_{\lambda}=T^{*} T \delta x-v
\end{gathered}
$$

as can be checked by direct calculation. Since $T^{*} T$ positive definite, $\left\|\left(P_{I} T^{*} T P_{I}\right)^{\dagger}\right\|$ is bounded independently of $x$ and $\lambda$, and thus so is $\left\|s[x, \lambda]^{-1}\right\|$. It follows that Theorem 5.4 is applicable and the following algorithm converges superlinearly if the initial guess is sufficiently close to the solution.

Algorithm 5.5 (Semi-smooth Newton Method)

- Choose $x_{0}, \lambda_{0} \in \mathbb{R}^{n}$.

- For $k=0,1, \ldots$ do:

1. Let $\mathcal{A}_{k}=\left\{i:\left(\lambda_{k}-c x_{k}\right)_{i}>0\right\} \subset\{1, \ldots, n\}$ and $\mathcal{I}_{k}=\mathcal{A}_{k}^{c}$.

2. Solve

$$
\begin{gathered}
T^{*} T x_{k+1}-\lambda_{k+1}=T^{*} y \\
\left.x_{k+1}\right|_{\mathcal{A}_{k}}=0 \\
\left.\lambda_{k+1}\right|_{\mathcal{I}_{k}}=0
\end{gathered}
$$

for $\left(x_{k+1}, \lambda_{k+1}\right)$, or equivalently

$$
\begin{gathered}
P_{\mathcal{I}_{k}} T^{*} T P_{I_{k}} x_{k+1}=P_{I_{k}} T^{*} y \\
x_{k+1}=P_{\mathcal{I}_{k}} x_{k+1} \\
\lambda_{k+1}=T^{*} T x_{k+1}-T^{*} y .
\end{gathered}
$$

Here, the operator $T^{*} T$ only has to be inverted on the inactive set $\mathcal{I}_{k}$.

3. Check stopping criterion (see below). 
The auxiliary constant $c>0$ can in principle be chosen freely. Choosing $c=\alpha$ usually leads to good results. The operator $P_{I_{k}} T^{*} T P_{I_{k}}$ in the first equation of the iteration step (5.13) is positive definite; therefore, the equation can be solved efficiently using the Conjugate Gradient (CG) method, which only requires applications of the forward operator.

\subsubsection{Duality gap as a stopping rule}

In Algorithm 5.5, $x_{k+1}$ and $\lambda_{k+1}$ only depend on $x_{k}$ and $\lambda_{k}$ through their dependence on $\mathcal{A}_{k}$. Since there are only finitely many possible subsets of $\{1, \ldots, n\}$ and since the algorithm converges, it actually converges in a finite number of steps. In principle, one could stop the iterations once the sets $\mathcal{A}_{k}$ and $\mathcal{A}_{k+1}$ are identical and obtain an exact solution (up to errors in solving the linear equation in each iteration). However, the running time of the algorithm can often be greatly improved by stopping earlier with an approximate solution. One possible way to estimate the distance to the exact solution is to consider the difference between the Tikhonov functional and a dual functional.

Definition 5.6. Let $C \subset \mathbb{R}^{n}$ be a non-empty, closed and convex cone, $F \in \mathbb{R}^{m \times n}, y \in \mathbb{R}^{m}$, $L \in \mathbb{R}^{k \times n}$ injective and $z \in \mathbb{R}^{k}$. The primal functional is defined as

$$
\mathcal{J}(x):=\frac{1}{2}\|F x-y\|^{2}+\frac{1}{2}\|L(x-a)\|^{2}+\chi_{C}(x)
$$

for $x \in \mathbb{R}^{n}$, and the dual functional is

$$
\mathcal{J}_{*}(p):=-\frac{1}{2}\|p\|^{2}+\langle p, y\rangle+\frac{1}{2}\|a\|_{L}^{2}-\frac{1}{2}\left\|P_{C}^{L}\left(\left(L^{*} L\right)^{-1} F^{*} p+a\right)\right\|_{L^{\prime}}^{2}
$$

for $p \in R^{m}$, where $\|\cdot\|_{L}=\|L \cdot\|$ and $P_{C}^{L}$ is the Euclidean projection onto $C$ in that norm. The problem

$$
x^{*}=\underset{x \in \mathbb{R}^{n}}{\operatorname{argmin}} \mathcal{J}(x)
$$

is called primal problem, and

$$
p^{*}=\underset{p \in \mathbb{R}^{m}}{\operatorname{argmax}} \mathcal{J}_{*}(p)
$$

is called the associated dual problem.

The relation between primal and dual problem is given by the following theorem.

Theorem 5.7. Problem (5.15) has a solution $x^{*}$, problem (5.16) has a solution $p^{*}$, and strong duality holds, i.e. $\mathcal{J}\left(x^{*}\right)=\mathcal{J}_{*}\left(p^{*}\right)$. $x^{*}$ and $p^{*}$ are related by

$$
\begin{gathered}
x^{*}=P_{C}^{L}\left(\left(L^{*} L\right)^{-1} F^{*} p^{*}+a\right), \\
p^{*}=y-F x^{*} .
\end{gathered}
$$




\section{Discretization and Implementation}

Proof. First, note that if we define the functional $\mathcal{R}$ by writing

$$
\mathcal{J}(x)=\frac{1}{2}\|F x-y\|^{2}+\mathcal{R}(x),
$$

then

$$
\mathcal{J}_{*}(p)=-\frac{1}{2}\|p\|^{2}+\langle p, y\rangle-\mathcal{R}^{*}\left(F^{*} p\right)
$$

where $\mathcal{R}^{*}$ is the conjugate of $\mathcal{R}$ as in Definition 3.10. Existence and uniqueness of the minimizer $x^{*}$ is shown in [EHN96, Theorem 5.15]. Let $\bar{p}:=y-F x^{*}$. Then $0 \in \partial \mathcal{J}\left(x^{*}\right)$ is equivalent to $F^{*} \bar{p} \in \partial \mathcal{R}\left(x^{*}\right)$. By Lemma 3.11, this implies $x^{*} \in \partial \mathcal{R}^{*}\left(F^{*} \bar{p}\right)$, from which

$$
0 \in-F x^{*}+F \partial \mathcal{R}^{*}\left(F^{*} \bar{p}\right)=\partial\left(-\mathcal{J}_{*}\right)(\bar{p})
$$

follows. So $\bar{p}=p^{*}$ is indeed the (unique) maximizer of $\mathcal{J}_{*}$. The first condition in (5.17) is the same as $F^{*} p^{*} \in \partial \mathcal{R}\left(x^{*}\right)$. Finally,

$$
\mathcal{J}\left(x^{*}\right)-\mathcal{J}_{*}\left(p^{*}\right)=-\left\langle p^{*}, F x^{*}\right\rangle+\mathcal{R}\left(x^{*}\right)+\mathcal{R}^{*}\left(F^{*} p^{*}\right)=0
$$

using Lemma 3.11 again.

Definition 5.8. The duality gap $\mathcal{G}$ at $(x, p) \in \mathbb{R}^{n} \times \mathbb{R}^{m}$ is defined as the difference between the primal and dual functional,

$$
\mathcal{G}(x, p):=\mathcal{J}(x)-\mathcal{J}_{*}(p) .
$$

Moreover, we define

$$
\mathcal{G}(x):=\mathcal{G}(x, y-F x)
$$

Written explicitly,

$$
\begin{gathered}
\mathcal{G}(x)=\frac{1}{2}\|x-r(x)\|_{L}^{2}-\frac{1}{2}\left\|r(x)-P_{C}^{L}(r(x))\right\|_{L^{\prime}}^{2} \\
r(x):=\left(L^{*} L\right)^{-1} F^{*}(y-F x)+a .
\end{gathered}
$$

Due to Theorem $5 \cdot 7, \mathcal{G}$ is always non-negative, and $\mathcal{G}\left(x^{*}\right)=0$. In addition, $\mathcal{G}(x)$ can be used to estimate the distance of $x$ to the solution of (5.15) in terms of the norm $\|\cdot\|_{L}$.

Theorem 5.9. For all $x \in C$ and $p \in \mathbb{R}^{m}$.

$$
\left\|L\left(x-x^{*}\right)\right\|^{2} \leq 2 \mathcal{G}(x, p)
$$

In particular, $\left\|L\left(x-x^{*}\right)\right\|^{2} \leq 2 \mathcal{G}(x)$.

Proof. If $x \notin C$, the statement is obvious since $\mathcal{J}(x)=\infty$. For $x \in C$, the optimality condition for the primal problem implies that

$$
\left\langle F^{*}(F x-y)+L^{*}(L x-a), x-x^{*}\right\rangle \geq 0 .
$$


Thus

$$
\begin{aligned}
\mathcal{J}(x)-\mathcal{J}\left(x^{*}\right) & =\frac{1}{2}\left\|F\left(x-x^{*}\right)\right\|^{2}+\frac{1}{2}\left\|L\left(x-x^{*}\right)\right\|^{2} \\
& +\left\langle F^{*}(F x-y)+L^{*}(L x-a), x-x^{*}\right\rangle \\
& \geq \frac{1}{2}\left\|L\left(x-x^{*}\right)\right\|^{2} .
\end{aligned}
$$

Since $\mathcal{J}\left(x^{*}\right) \geq \mathcal{J}_{*}(p)$ for all $p \in \mathbb{R}^{m}$ by Theorem $5 \cdot 7$, the assertion follows.

This leads to the following stopping criterion for Algorithm 5.5:

3. Stop the iteration if $\mathcal{A}_{k+1}=\mathcal{A}_{k}$, or if $\mathcal{G}\left(P_{C} x_{k+1}\right) \leq \varepsilon$ for some small $\varepsilon>0$. Return $P_{C} x_{k+1}$.

$P_{C}$ is the projection onto the constraint set. We use $P_{C} x_{k+1}$ instead of $x_{k+1}$, since the latter will in often violate the constraint, and thus the duality gap may be infinite at $x_{k+1}$. This would make the stopping criterion fail even in cases where $x_{k+1}$ is close to $x^{*}$.

To apply this stopping rule to problem (5.2), in principle we could take

$$
F=\sqrt{G^{Y}} B, \quad y=\sqrt{G^{Y}} z^{\delta}, \quad L=\sqrt{\alpha G^{X}}, \quad a=0
$$

and $C=\left\{x \in \mathbb{R}^{N_{x} \times N_{u}}: x \geq 0\right\}$. One would then have to evaluate the projection onto $C$ in the $G^{X}$-norm, the computation of which may itself not be straightforward.

To overcome this problem, we write $G^{X}$ as

$$
\alpha G^{X}=\beta \mathbb{1}+D^{*} D .
$$

for some sufficiently small $\beta>0$ and $D \in \mathbb{R}^{n \times n}$, which can always be achieved since $G^{X}$ is positive definite. If we now put

$$
F=\left(\begin{array}{c}
\sqrt{G^{Y}} B \\
D
\end{array}\right), \quad y=\left(\begin{array}{c}
\sqrt{G^{Y}} z^{\delta} \\
0
\end{array}\right), \quad L=\sqrt{\beta}, \quad a=0,
$$

then $P_{C}^{L}=P_{C}$ can be evaluated cheaply. The dual functional using (5.19) is

$$
\mathcal{J}_{*}(p)=-\frac{1}{2}\|p\|^{2}+\langle p, y\rangle-\frac{1}{2 \beta}\left\|P_{C}\left(F^{*} p\right)\right\|^{2}
$$

so for the evaluation of $\mathcal{G}(x)=\mathcal{J}(x)-\mathcal{J}_{*}(y-F x)$, only $F^{*} y=B^{*} G^{Y} z^{\delta}$ and

$$
F^{*} F=B^{*} G^{Y} B+D^{*} D=B^{*} G^{Y} B+\alpha G^{X}-\beta \mathbb{1}
$$


are required, not the auxiliary operator $D$. Note that (5.19), combined with Theorem 5.9 and the stopping rule, leads to

$$
\frac{\beta}{2}\left\|x-x^{*}\right\|^{2} \leq \varepsilon
$$

whereas (5.18) would yield the stronger estimate

$$
\frac{\alpha}{2}\left\|\sqrt{G^{X}}\left(x-x^{*}\right)\right\|^{2} \leq \varepsilon
$$

Since we are working in the finite dimensional setting, the norms are of course equivalent; numerically, they can still differ considerably. This has to be accounted for by choosing a smaller tolerance $\varepsilon$.

\subsection{Spherical Harmonics basis on $S^{2}$}

As alternate choice of basis on $S^{2}$, the SHs from (4.14),

$$
Y_{l m}(u)=\sqrt{\frac{2 l+1}{4 \pi} \frac{(l-m) !}{(l+m) !}} P_{l}^{m}\left(u_{3}\right)\left(\frac{u_{1}+i u_{2}}{\left|u_{1}+i u_{2}\right|}\right)^{m}
$$

for $l=1,2, \ldots$ and $-l \leq m \leq l$ can be used. More precisely, we use a modified basis as introduced in [DAFDo7], consisting of real-valued linear combinations of SHs of even order $l$, the latter due to symmetry assumptions, the former to avoid having to handle unphysical complex-valued ODFs. For simplicity, the basis functions are numbered by a single index $j \geq 1$ instead of the tuple $(l, m)$.

$$
Y_{j}:= \begin{cases}\sqrt{2} \operatorname{Re}\left(Y_{l(j), m(j)}\right), & m(j)<0 \\ Y_{l(j), 0}, & m(j)=0 \\ \sqrt{2} \operatorname{Im}\left(Y_{l(j), m(j)}\right), & m(j)>0\end{cases}
$$

This basis is widely used in DW-MRI, not only for spherical deconvolution, but also for models like Q-Ball (2.14) and solid-angle Q-Ball (2.16). The reason is that it greatly simplifies the various forward operators. In fact, the basis diagonalizes all rotationally invariant operators, in particular also the Laplace-Beltrami operator. Convergence of the discretizing projection $P_{h}$ that is obtained from cutting off this basis at a particular order is obvious from the definition of $H_{\text {hor }}$.

The main downside of the basis is a more complicated positivity constraint. As for the spatial basis, the constraint is approximated by checking it only on a finite subset $\left\{u_{i}\right\}_{i=1}^{n_{c}} \subset S^{2}$. In the context of spherical deconvolution, this form of the constraint was first suggested in [TCCo7], where the constraint is checked on more points than there are unknown coefficients or data points, which enables super-resolution of the 
reconstructed ODFs, i.e the number of reconstructed $\mathrm{SH}$ coefficients is larger than the number of data points. In the following, we will therefore always assume that the matrix $H \in \mathbb{R}^{n_{c} \times n}$,

$$
H_{i j}=Y_{j}\left(u_{i}\right)
$$

is injective. As for the matrix $B$ implementing the forward operator, we will identify $H$ with $\mathbb{1} \otimes H$ if no confusion arises.

If we approach this problem along the lines of the semi-smooth Newton method used above, we have to solve problem of the general form

$$
\underset{H x \geq 0}{\operatorname{argmin}}\|T x-y\|^{2} \text {. }
$$

Lagrange multipliers for this problem exist if the constraint is feasible, which clearly is the case since $Y_{00}>0$. Proceeding similar to the case above, this leads to the iteration

$$
\begin{gathered}
\mathcal{A}_{k}=\left\{i:\left(\lambda_{k}-c H x_{k}\right)_{i}>0\right\}, \quad \mathcal{I}_{k}=\mathcal{A}_{k^{\prime}}^{c} \\
T^{*} T x_{k+1}-H^{*} \lambda_{k+1}=T^{*} y \\
\left.H x_{k+1}\right|_{\mathcal{A}_{k}}=0 \\
\left.\lambda_{k+1}\right|_{\mathcal{I}_{k}}=0,
\end{gathered}
$$

In contrast to the iteration in Algorithm 5.5, the linear system can not be simplified by restricting to the inactive set and eliminating $\lambda$. So one has to resort to solving the complete system, which may be significantly larger. Furthermore, it is not positive definite, making other solvers like GMRES or BiCGSTAB instead of CG necessary. Finally, depending on the inactive set, the system may not even be uniquely solvable if $H$ is not surjective. Numerical experiments using GMRES indeed showed poor convergence.

An alternative route is taken in [TCCo7], where the constraint is only implemented approximately but in a way that can be computed much easier. Given $x_{k}$, let the negative set $\mathcal{N}_{k}$ be defined by $\mathcal{N}_{k}:=\left\{i:\left(H x_{k}\right)_{i}<0\right\}$. Then the iteration consists in solving

$$
\underset{x \in \mathbb{R}^{n}}{\operatorname{argmin}}\left(\|T x-y\|^{2}+\sigma\left\|P_{\mathcal{N}_{k}} H x\right\|^{2}\right), \quad \sigma>0
$$

i.e. the constraint is implemented by penalizing the solution on points where the previous iterate violated it. The iterations are stopped at the first $k$ for which $\mathcal{N}_{k}=$ $\mathcal{N}_{k-1}$.

In [TCCo7], the method is suggested on an ad-hoc basis. It can, however, be interpreted as Moreau-Yosida regularization of the constraint $H x \geq 0$. Moreau-Yosida regularization proceeds by replacing the indicator function $\chi_{\geq 0}(H x)$ that is implicit in (5.20) with its Moreau envelope

$$
\inf _{w \in \mathbb{R}^{n_{\mathcal{C}}}}\left(\chi_{\geq 0}(H x-w)+\sigma\|w\|^{2}\right),
$$




\section{Discretization and Implementation}

leading to the relaxed problem

$$
\underset{H x \geq w}{\operatorname{argmin}}\left(\|T x-y\|^{2}+\sigma\|w\|^{2}\right)
$$

where minimization is now performed over both $x$ and the auxiliary variable $w$. The necessary and sufficient first order optimality conditions for (5.22) can be written as

$$
\begin{gathered}
T^{*} T x-H^{*} \lambda=T^{*} y \\
\sigma w+\lambda=0 \\
\lambda=\max (0, \lambda-\sigma(H x-w)),
\end{gathered}
$$

which is the same as

$$
\begin{gathered}
T^{*} T x+\sigma H^{*} \min (0, H x)=T^{*} y \\
w=-\sigma^{-1} \lambda .
\end{gathered}
$$

A semi-smooth Newton step for the first equation consists in solving

$$
T^{*} T x+\sigma H^{*} P_{\mathcal{N}_{k}} H x=T^{*} y,
$$

which is just the optimality condition for (5.21).

Convergence of the Moreau-Yosida regularization for $\sigma \rightarrow \infty$ has been investigated in [IKoo]. We will use this algorithm even though it yields only an approximation to the original problem, since this allows for comparison of the regularized method with the constrained spherical deconvolution method from [TCCo7] — which is included as the case $\alpha=0-$, and since it can be implemented easily and efficiently.

Finally, the Gramian matrix can be computed similarly to Section 5.2 by numerical quadrature on $S^{2}$, simply replacing the functions $\varphi_{u}$ with $Y_{j}$. Quadrature points and weights can be obtained for example by using linear interpolation as described above. Since this is all done in a precomputation step, the number of points can be chosen as high as needed to achieve the desired accuracy.

\subsubsection{Duality gap}

As above, we would like to use the duality gap to stop the iterations earlier with an approximate solution. For this, split $G^{X}$ as

$$
\alpha G^{X}=\beta H^{*} H+D^{*} D
$$

We then write the functional in (5.22) as (5.14) using the definitions

$$
F=\left(\begin{array}{cc}
\sqrt{G^{Y}} B & 0 \\
D & 0
\end{array}\right), \quad y=\left(\begin{array}{c}
\sqrt{G^{Y}} z^{\delta} \\
0
\end{array}\right), \quad L=\left(\begin{array}{cc}
\sqrt{\beta} H & 0 \\
0 & \sqrt{c} \mathbb{1}
\end{array}\right), \quad a=0
$$


and $C=\{(x, w): H x \geq w\}$, and have to compute an expressions of the form

$$
\begin{aligned}
L P_{C}^{L}\left(\begin{array}{l}
r_{1} \\
r_{2}
\end{array}\right) & =\underset{H x \geq w}{\operatorname{argmin}}\left(\beta\left\|H\left(x-r_{1}\right)\right\|^{2}+c\left\|w-r_{2}\right\|^{2}\right) \\
& =\left(\begin{array}{cc}
\sqrt{\beta} & 0 \\
0 & \sqrt{c}
\end{array}\right) \underset{\substack{v \geq w \\
v \in \mathcal{R}(H)}}{\operatorname{argmin}}\left(\beta\left\|v-H r_{1}\right\|^{2}+c\left\|w-r_{2}\right\|^{2}\right) .
\end{aligned}
$$

This is difficult to solve explicitly due to the range condition $v \in \mathcal{R}(H)$, but can be computed numerically using Dykstra's algorithm [Dyk83], which calculates the projection $P_{C_{1} \cap C_{2}} x$ of a point $x$ onto the intersection of two closed, convex sets $C_{1}$ and $C_{2}$ by applying $P_{C_{1}}$ and $P_{C_{2}}$ only. The algorithm is

Algorithm 5.10 (Dykstra's Algorithm)

- Set $x_{0}=x, a_{0}=b_{0}=0$.

- For $k=1,2, \ldots$, set

$$
\begin{aligned}
y_{k} & =P_{C_{1}}\left(x_{k-1}+a_{k-1}\right) \\
a_{k} & =x_{k-1}+a_{k-1}-y_{k} \\
x_{k} & =P_{C_{2}}\left(y_{k}+b_{k-1}\right) \\
b_{k} & =y_{k}+b_{k-1}-x_{k} .
\end{aligned}
$$

It was shown in [Dyk83, Theorem 3.1] that if $C_{1}$ and $C_{2}$ are closed, convex cones with non-empty intersection, then $x_{k}$ converges to $P_{C_{1} \cap C_{2}} x$ as $k \rightarrow \infty$. In fact, the algorithm is known to converge in much more general cases.

So to evaluate the projection, we only have to project onto $\mathcal{R}(H)$ and evaluate expressions of the form

$$
\underset{v \geq w}{\operatorname{argmin}}\left(\beta\left\|v-v_{0}\right\|^{2}+c\left\|w-w_{0}\right\|^{2}\right)
$$

The $i$-th components of the minimizer $(v, w)$ are explicitly given by

$$
v_{i}=v_{0, i}, \quad w_{i}=w_{0, i} \quad \text { if } \quad v_{0, i} \geq w_{0, i}
$$

and

$$
v_{i}=w_{i}=\frac{\beta v_{0, i}+c w_{0, i}}{\beta+c} \quad \text { otherwise. }
$$

Dykstra's algorithm is a first-order method, so convergence can be rather slow and require a large number of steps. In that case, even simple operations like the ones above can lead to computation times that are too large for a stopping criterion. Note however that all of the steps above can be trivially parallelized, voxel-wise for the range 
projection and pointwise for the inequality constraint. Moreover, a parallel version of Dykstra's algorithm is available [Comog]. Therefore, it should be possible to implement computation of the duality gap as fast as needed.

Finally, the error can be estimated from the duality gap as

$$
\frac{\beta}{2}\left\|H\left(x-x^{*}\right)\right\|^{2} \leq \frac{1}{2}\left\|L\left(\begin{array}{c}
x-x^{*} \\
w-w^{*}
\end{array}\right)\right\|^{2} \leq \mathcal{G}\left(\begin{array}{c}
x \\
w
\end{array}\right)
$$

where $w$ can be computed using $(5.23)$ as $w=\min (0, H x)$. Since the SHs are orthonormal, the discretized matrix $H$ should be close to an isometry if the points $\left\{u_{i}\right\}$ are chosen properly, possibly after inclusion of appropriate non-negative quadrature weights, so the inequality above can be read as an approximate bound for $\left\|x-x^{*}\right\|$. 


\section{Numerical Experiments}

In this chapter, the methods developed in the preceding chapters will be tested on simulated, phantom and in-vivo data. We will compare the performance of purely $L^{2}$-regularized reconstructions with the $L^{2}+D_{\text {hor }}$ penalty (4.11) and the $L^{2}+D_{\text {hor }}+$ Grad penalty (4.12), as well as the two choices of basis on $S^{2}$ introduced in Sections 5.2 and 5.4.

One of the main problems in ODF reconstruction is that there is neither a canonical measure of the quality of an ODF, nor of the distance between two ODFs, that accurately captures the features that a "good" ODF reconstruction should have. Therefore, in most parts of this chapter, only graphical depictions of reconstructed images will be shown. Assessing the performance of the various methods under investigation then has to be performed in a more qualitative fashion by visual inspection. Moreover, for the phantom data, where the ground truth of the fiber tracks is known, some tracking results will be shown to highlight the effect of both smoothing and artifacts introduced by the spatial regularization.

ODF reconstructions will be depicted by displaying, for each voxel of a 2-dimensional slice of the reconstruction, a glyph constructed by deforming a sphere according to the value of the ODF in the respective direction. So in voxel $x$, the surface

$$
\left\{\psi(x, u) u: u \in S^{2}\right\}
$$

is displayed. The main diffusion directions can be read of by the "spikes" of the resulting glyph. The background of the images will show the corresponding GFA maps.

\subsection{Convergence of the projections}

We first numerically test the convergence of the discretizing projections $P_{h}$ in $H_{\text {hor }}$. Let $\iota: H_{\text {hor }} \rightarrow L^{2}$ be the embedding. Then we want to investigate the norm convergence $\left\|\iota \circ P_{h} \rightarrow \iota\right\| \rightarrow 0$ as $h \rightarrow 0$. The problem here is that for $\psi \in H_{\text {hor }}$, one needs to quantify $\psi-P_{h} \psi$, i.e. its component outside of the discrete subspace. We will do this approximately by choosing a sufficiently fine discretization $X_{0} \subset H_{\text {hor }}$ in place of the full infinite-dimensional space, and consider a sequence $X_{h} \subset X_{0}$ of coarser discrete spaces approaching the fine space. 


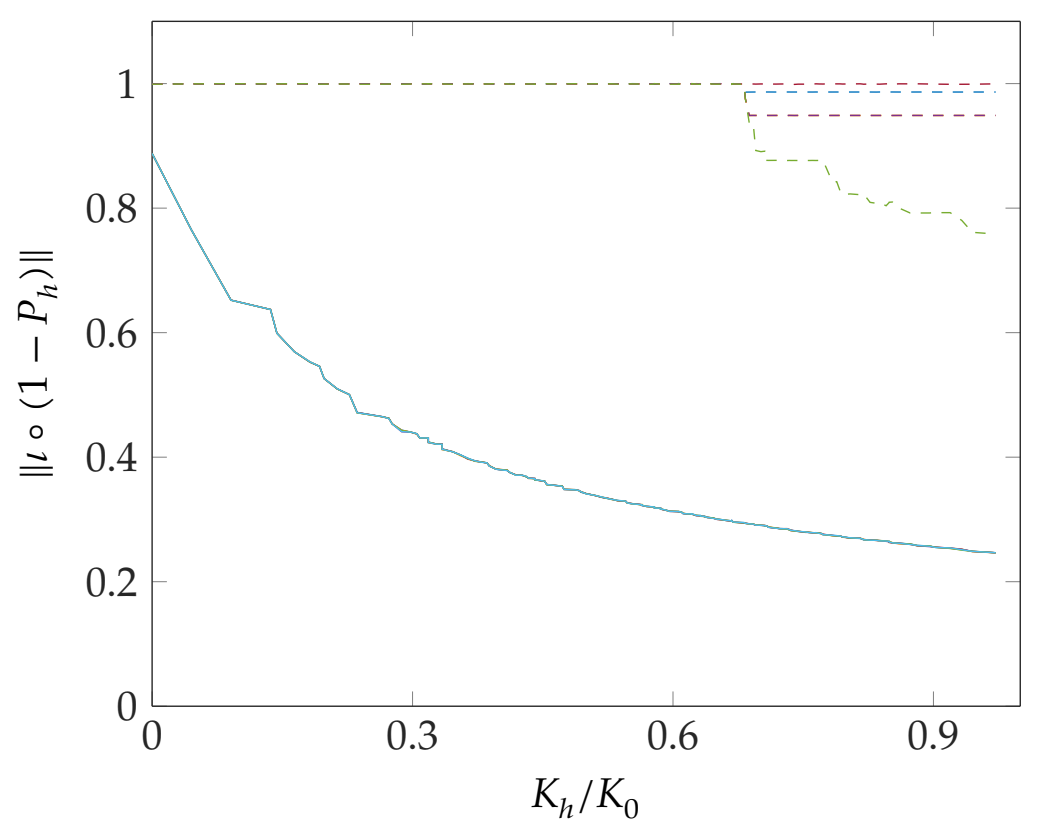

Figure 6.1: Convergence of the projection $P_{h}$ viewed as a map $H_{\text {hor }} \rightarrow L^{2}$ ( $\iota$ is the embedding) plotted against the spatial cutoff frequency for even $\mathrm{SH}$ orders $10 \leq$ $L_{h} \leq 20$. (Solid): Including the angular regularization term (4.12) (the curves are almost indistinguishable). (Dashed): Without the angular regularization term (4.11); $\mathrm{SH}$ order is increasing from bottom up.

The linear basis functions as introduced in Chapter 5 would pose a problem in this setting, since the discrete space constructed for a coarse set of points on $S^{2}$ is not generally a subspace of the discrete space for a refined point set. This would lead to additional technical difficulties that are not present in the continuous setting we try to approximate.

Therefore, we only investigate projections for $\mathrm{SH}$ basis functions of the form $\xi_{k} \otimes Y_{l m}$ as defined in Chapter 5 , and choose a discrete space of the form $X_{h}:=\operatorname{span}\left\{\xi_{k} \otimes Y_{l m}:\|k\| \leq\right.$ $\left.K_{h}, l \leq L_{h}\right\}$. The fine discretization $X_{0}$ is defined similarly, for sufficiently large cutoff parameters $K_{0}, L_{0}$. Then $P_{h}$ and the norm above can be evaluated numerically by taking advantage of the fact that the Gramian matrices $G_{0}$ and $G_{h}$ of $X_{0}$ and $X_{h}$ decouple with respect to the spatial part $\xi_{k}$ of the basis functions. The projection has the explicit form $P_{h}=G_{h}^{\dagger} G_{0}$, while the norm $\left\|\iota \circ P_{h} \rightarrow \iota\right\| \rightarrow 0$ can be computed by a power iteration.

In Figure 6.1, the results for various $L_{h}$ are plotted against the spatial frequency cutoff $K_{h}$ for norms with the additional Laplace-Beltrami operator, clearly showing convergence almost independently of the chosen $L_{h}$. The figure also shows the results of the same numerical test when omitting the Laplace-Beltrami penalty. While these also decrease for large enough $K_{h}$, the effect becomes weaker as the SH order $L_{h}$ increases. This can likely be attributed to the fact that the finite $\mathrm{SH}$ order $L_{h}$ itself provides some angular smoothness (i.e. "regularization by discretization"). Therefore, for fixed $L_{h}$, 
convergence of $P_{h} \equiv P_{L_{h}, K_{h}}$ to $P_{L_{h}, \infty}$ as $K_{h} \rightarrow \infty$ is possible, but the convergence speed deteriorates with increasing $L_{h}$, such that the combined limit $h \rightarrow 0 \Longleftrightarrow K_{h}, L_{h} \rightarrow \infty$ does not exist. This may also explain why in practice, when choosing a fixed and not too large $L_{h}$, satisfactory reconstructions can also be obtained without explicit angular regularization.

\subsection{Reconstructions of phantom data and comparison of bases}

In this section, we compare reconstructions performed on phantom data ${ }^{1}$ using various choices of the regularization parameters and for both choices of bases described in Chapter 5 .

Construction of the phantom and details on the acquisition are described in more detail in [FDG+11]. The data was sampled on a $64 \times 64 \times 3$ spatial grid with $3 \mathrm{~mm}$ isotropic voxel spacing, using 64 gradient directions and $b=1500 \mathrm{~s} / \mathrm{mm}^{2}$, with each gradient being measured twice to reduce noise. The structure of the phantom is shown in Figure 6.2, together with three regions of interest - a 90 degree crossing, a $~ 70$ degree crossing and a curved structure. Reconstructions were performed on the whole data, without employing a mask.

As forward model, the spherical unscaled convolution model (2.17) was used, with convolution kernel parametrized by SHs. This makes the forward operator in the $\mathrm{SH}$ basis particularly simple. The coefficients of the convolution kernel up to order 8 were estimated from the data using the MRtrix software package ${ }^{2}-$ higher order $^{2}$ coefficients turned out to be small enough to be neglected. The estimation proceeds essentially by averaging the data over all voxels in which a DTI reconstruction shows an FA value exceeding a given threshold - since these are assumed to contain only a single fiber each - and accounting for rotation of the data as indicated by the main diffusion direction obtained from the diffusion tensor.

For the SH basis, the ODF was reconstructed in SHs up to order 12, i.e. with 91 unknown coefficients in each voxel. This means that SH coefficients of order 10 and 12 are determined solely by the regularization penalty and the non-negativity constraint. The constraint was checked on a set of 246 points constructed as $n=7$ subdivision of the icosahedron as in in (5.6), using only half of the resulting symmetric point set since the other half is redundant due to the symmetry of the $\mathrm{SH}$ basis.

\footnotetext{
${ }^{1}$ Mechanical conception and data acquisition of the hardware phantom were performed by Cyril Poupon, Laurent Laribière, Grégory Tournier, Denis Fournier, Jérémy Bernard and Irina Kezele for the MICCAI conference 2009. The data were obtained from the Laboratoire de Neuro-imagerie Assisté par Ordinateur (LNAO). See also http://www. Inao.fr/spip.php?rubrique79.

${ }^{2}$ Available at http://www.brain.org.au/software/. Developed at Brain Research Institute, Melbourne, Australia. Further information can also be found in in [TCC12].
} 


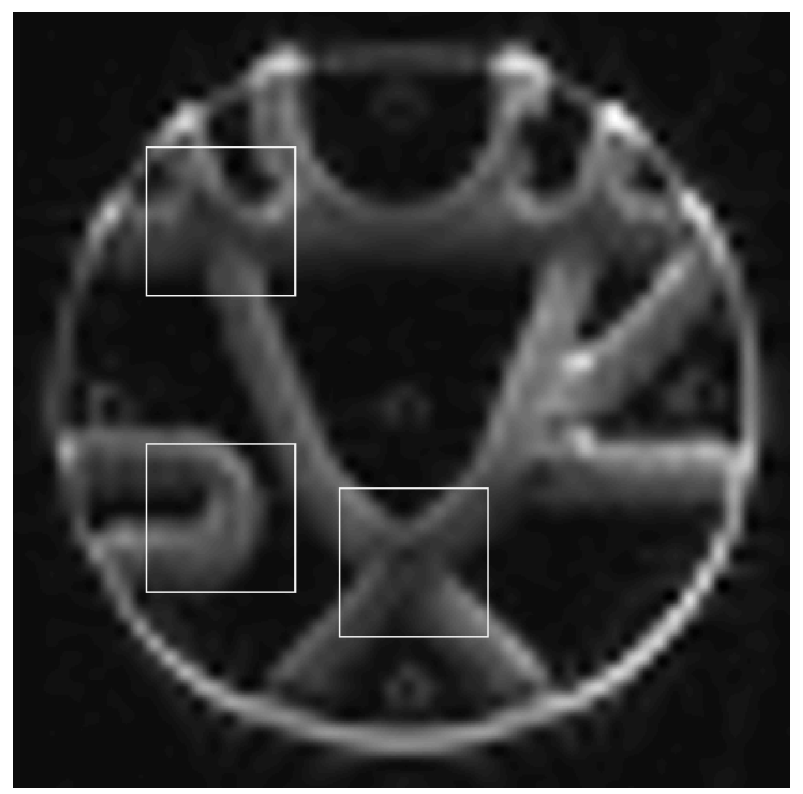

Figure 6.2: Structure of the physical phantom $(b=0$ image) with three regions of interest. ODF reconstructions of these regions are in Figures 6.3 and 6.4.

For the linear basis, the same point set on the half-sphere was chosen. Symmetry of the ODFs was enforced by constructing the basis functions $\varphi_{u}$ in (5.5) for the full point set and using a basis of the form $\left\{\varphi_{u}+\varphi_{-u}\right\}$ in the implementation. Note that the SH reconstruction has fewer unknown coefficients than the linear case. This is due to better approximation properties of the SHs.

Results are shown in Figures 6.3 and 6.4 using three regularization penalties: a pure $L^{2}$-penalty, an additional spatial $D_{\text {hor }}$-penalty, and finally including the angular derivative Grad that was crucial in obtaining the convergence result (4.18). Regularization parameters were chosen identically for corresponding reconstructions in SH and linear basis. Running times for reconstructions in the linear basis were typically slower by a factor of $\sim 3$.

For the crossing regions, the spatial penalty clearly improves coherence and resolution of the crossings compared to the $L^{2}$ reconstruction, in particular for the more noisy 70 degree crossing. For the curved structure, the spatial penalty causes some visible artifacts tangential to the structure. These are reduced somewhat by the additional angular penalty, essentially by blurring the spurious crossings. This of course also limits the achievable angular resolution. Therefore, the corresponding regularization parameter should not be chosen too large. Effects of these artifacts on fiber tracking performed on the reconstructed ODFs will also be discussed in the next section.

Concerning the choice of basis, both result in visually very similar reconstructions. For the $L^{2}+D_{\text {hor }}$ case, the linear basis seems a little more susceptible to noise, in particular in the edge areas of the crossings. This may be due to the implicit smoothness of the 
$\mathrm{SH}$ basis already mentioned in the previous section. Moreover, the corresponding GFA maps show some GFA "leaking" into areas outside of the phantom by prolonging elongated structures a bit too far. This effect is also more pronounced in the linear basis.

Maxima of ODFs in crossing areas appear smaller than in single-fiber voxels in both bases for not entirely clear reasons, and therefore are susceptible to over-blurring by the angular regularization. A possible explanation for this are scaling issues associated to $\psi$ being a density, similar to what was discussed below (2.20). It may also be due to the fact that we actually reconstruct the product $\rho \psi$ in the unscaled version of the convolution (2.17), and the unweighted image $\rho$ (Figure 6.2) shows a visibly decreased intensity in crossing areas. This is likely an effect specific to this particular phantom, since the presence of more fibers in these areas leads to a measurable decrease in water density. Another possible explanation is the unrealistic monotonic behavior of the spherical convolution as noted in the remark at the end of Section 2.2.3. Note also that the in-vivo images below (Figures 6.12 and 6.13) show similar scaling behavior. This is especially problematic since it contradicts the smoothness assumptions used in the design of the horizontal penalty term.

\subsubsection{Artifacts and tracking}

To illustrate the performance of the regularization penalty and highlight the effect of the introduced artifacts, Figures 6.5 and 6.6 show some tracking results on the same reconstructions as above. The algorithm was developed by Helen Schomburg [Sch15] and is a variant of the classic FACT algorithm [XZC+99] for tracking nerve fibers from DTI reconstructions, adapted to the more complex setting of ODF reconstructions. The plots were produced using only the most basic variant of the algorithm. Here, a track is computed as a sequence of positions $\left(x_{k}\right) \in \Omega$ and orientations $\left(u_{k}\right) \in S^{2}$ as follows:

Algorithm 6.1 (Simple Tracking)

- Pick a step length $\lambda>0$ and an angle $\nu>0$.

- Start at a seed point $x_{0} \in \Omega$ that is likely to contain a single fiber, and choose the maximum $u_{0}$ of the ODF at that point, $u_{0}=\operatorname{argmax} \psi(x, \cdot)$, as starting direction.

- In each step $\left(x_{k}, u_{k}\right)$, estimate $\psi\left(x_{k}, u\right)$ by trilinearly interpolating $\psi\left(x_{k}^{i}, u\right)$ from the surrounding grid points $\left\{x_{k}^{i}\right\}_{i=1}^{8}$ for each for $u \in S^{2}$ separately.

- Let $u_{k+1}=\operatorname{argmax}_{\Varangle\left(u, u_{k}\right) \leq \nu} \psi\left(x_{k}, u\right)$.

- Let $x_{k+1}=x_{k}+\lambda u_{k+1}$.

- Stop if entering a region that has been predetermined to not contain any fibers (for example based on the GFA value or on prior knowledge). 

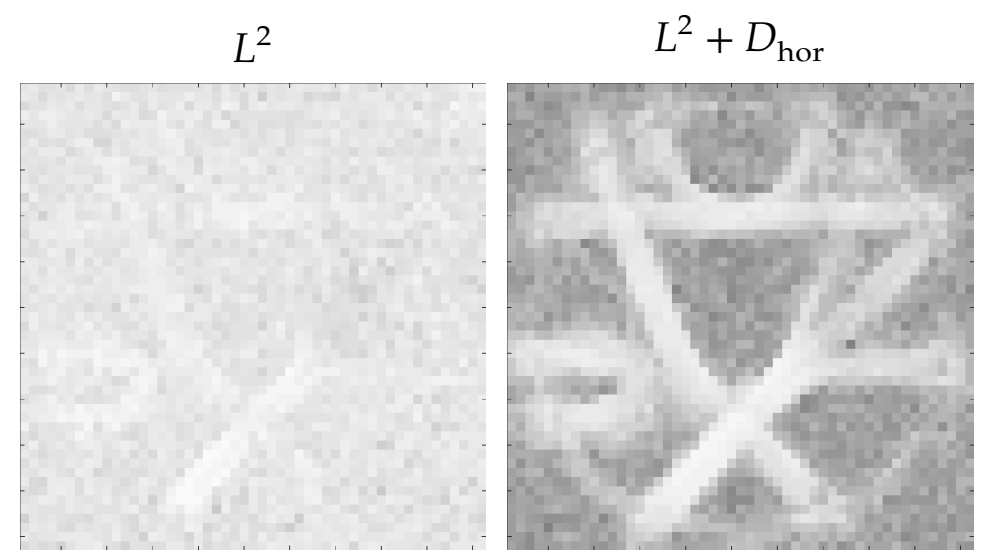

$L^{2}+D_{\text {hor }}+$ Grad
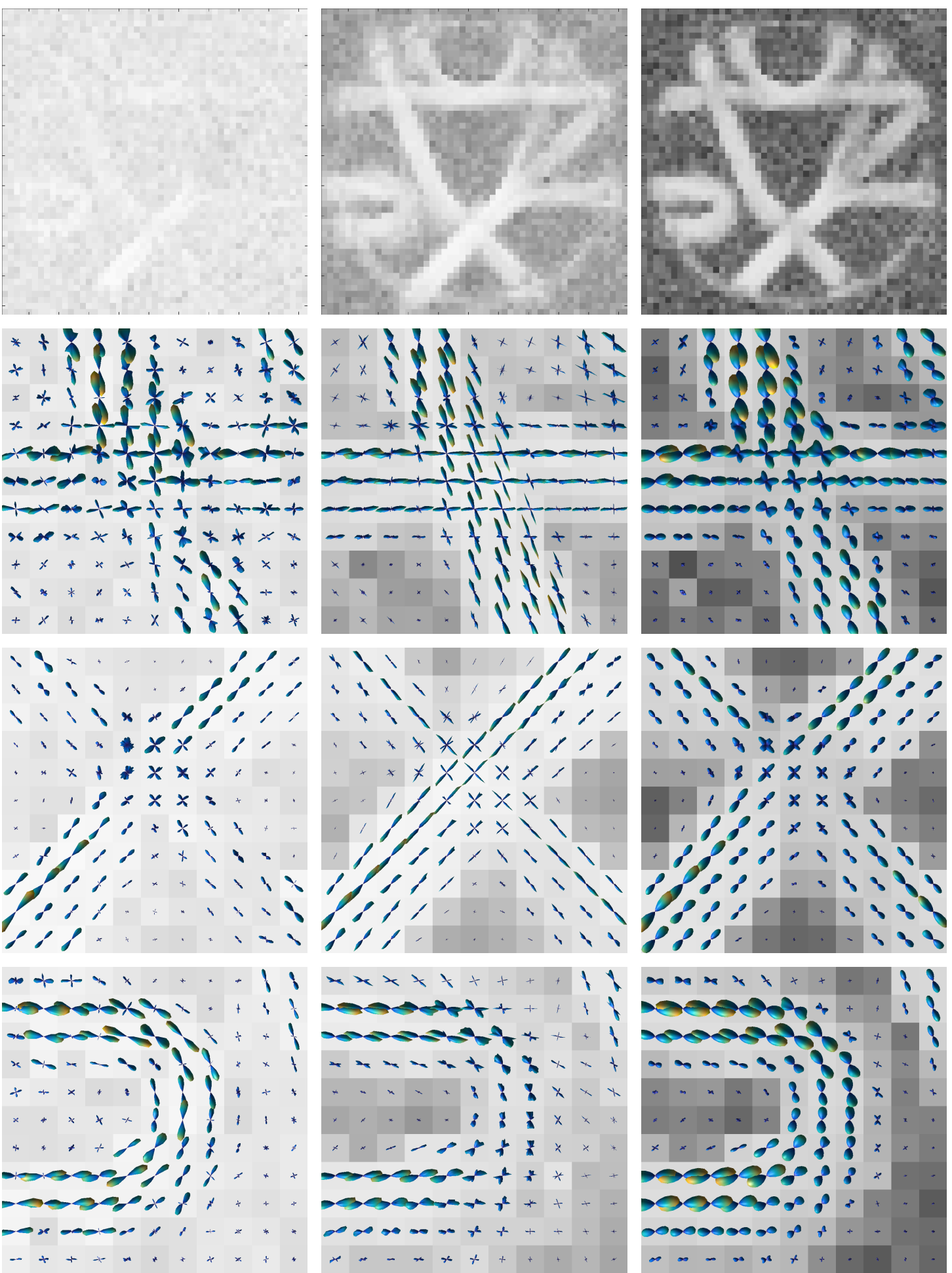

Figure 6.3: ODF reconstructions for the highlighted regions of the phantom in Figure 6.2 using the spherical convolution model in the linear basis, for various choices of regularizing penalties. The top row shows the GFA map. 

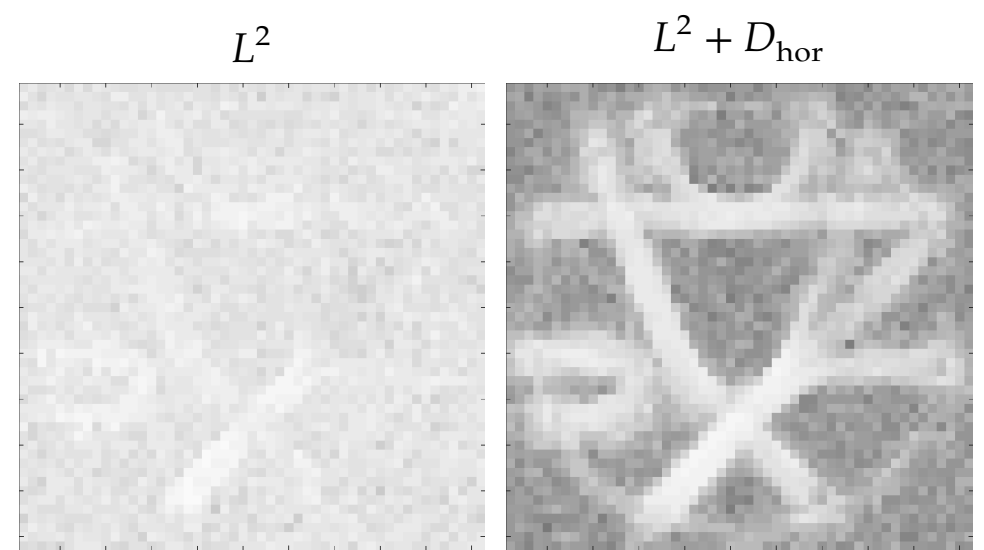

$$
L^{2}+D_{\text {hor }}+\text { Grad }
$$
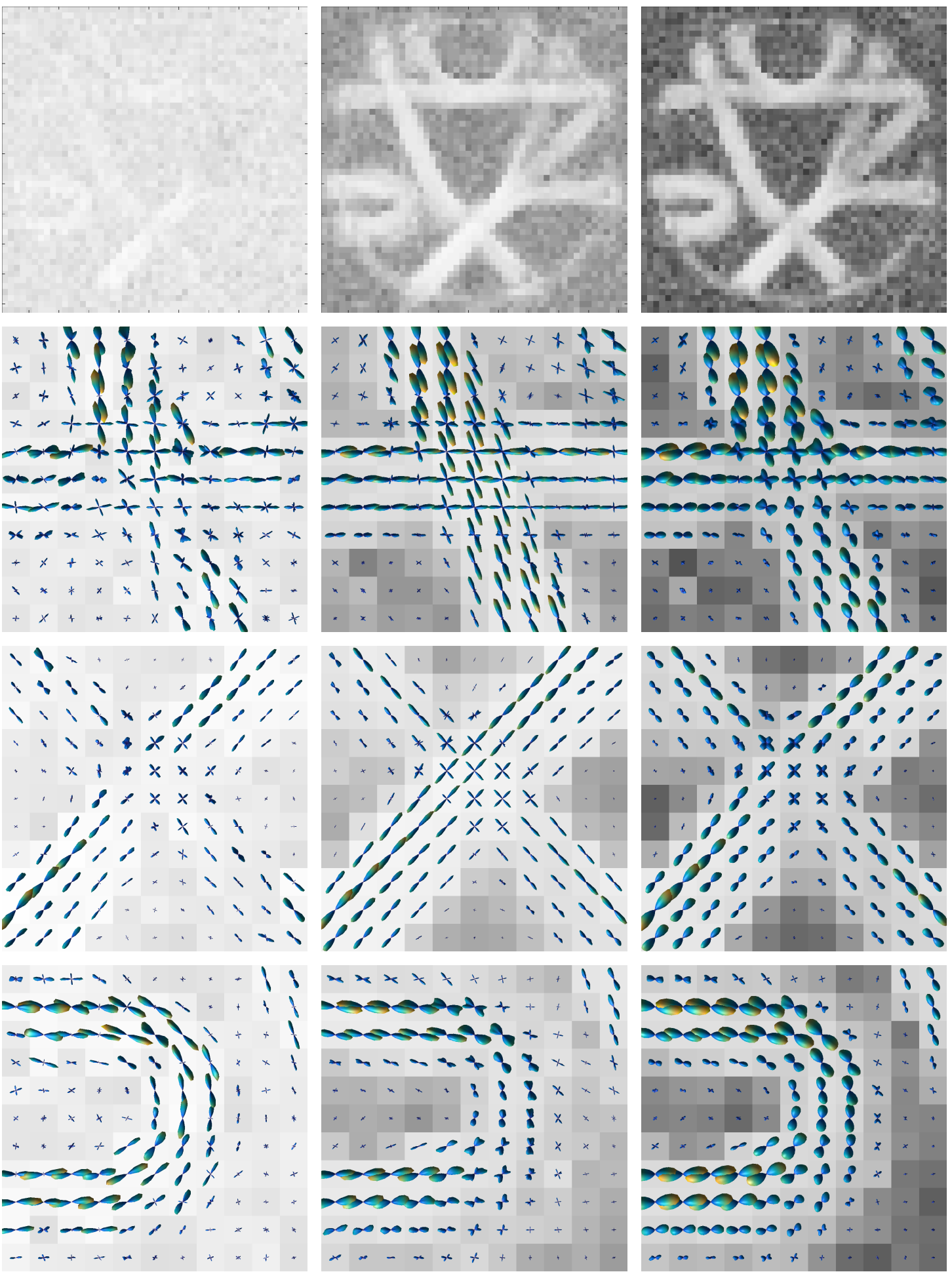

Figure 6.4: ODF reconstructions for the highlighted regions of the phantom in Figure 6.2 using the spherical convolution model in the SH basis, for various choices of regularizing penalties. The top row shows the GFA map. 
More advanced versions of this algorithm of course lead to better result and are able to track successfully through all of the highlighted regions. However, that is outside the scope of this thesis.

While the results in all cases are good for the 90 degree crossing, the algorithm is often not able to track through the non-perpendicular crossing for the reconstruction without spatial penalty, since the poor SNR in that region leads to too much spatial incoherence. An additional $D_{\text {hor }}$-penalty leads to significant improvements in this area, i.e. for straight fibers. On the other hand, it shows characteristic weaknesses in the curved region. While these are improved by the additional Grad-penalty, this also leads to some tracks taking the wrong turn in straight crossings, in particular for the obtuse angle. Still, the number of stopping tracks is significantly reduced.

Confirming the visual impression above, the $\mathrm{SH}$ reconstruction is slightly smoother, leading to better results in the curved structure without Grad-penalty while at the same time causing more tracks to deviate in the straight crossings.

\subsubsection{Non-negativity constraint}

In Figure 6.7, unconstrained reconstructions of the same data as above using the $\mathrm{SH}$ basis are displayed to investigate the necessity of the non-negativity constraint. For $L^{2}$-regularization, the constrained significantly improves the results, but if other types of regularization are included, the advantage almost vanishes. With $D_{\text {hor }}$-penalty, differences are almost only visible in the GFA map, while with additional Grad-penalty, there is hardly any visually noticeable difference. Reconstruction with only $L^{2}$ - and Grad-penalties, which were omitted for brevity's sake, show similarly satisfactory results without the constraint, so the effect does not necessarily depend on spatial regularization, but on sufficient suppression of the noise. For situation where fast reconstructions are required, it may therefore be possible to omit the constraint, trading off some accuracy for computational time.

\subsection{Simulated data}

To test convergence of the method, we simulated ODFs as depicted in Figure $6.8-\mathrm{a}$ straight crossing and a curved structure - using a multi-tensor model on a $50 \times 50 \times 3$ spatial grid. Corresponding data was simulated using the spherical convolution model, with kernel taken from the phantom model above for simplicity, using 64 gradient directions. The data was subsequently perturbed by Gaussian noise with noise level varying between $1 \%$ and $20 \%$, i.e. the standard deviation of the noise was chosen as the given percentage of the standard deviation of the simulated data. Then reconstructions were performed from the noisy data using SHs up to order 10 as basis, for various 

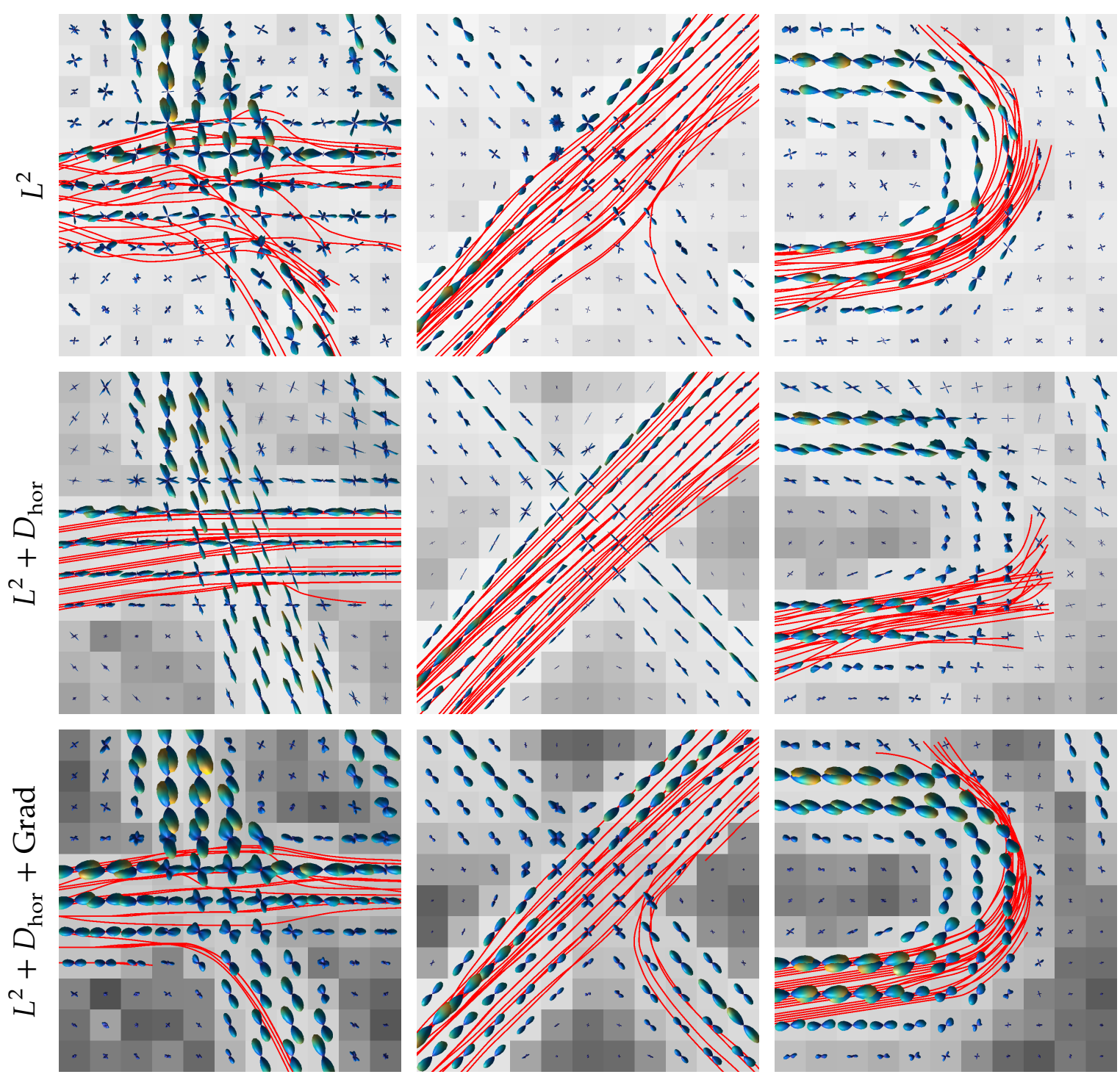

Figure 6.5: Tracking results using Algorithm 6.1 for the spherical deconvolution reconstructions in the linear basis shown in Figure 6.3. The seed regions are: (left) at the left edge, (center) at the top right corner, (right) at the bottom left edge. 

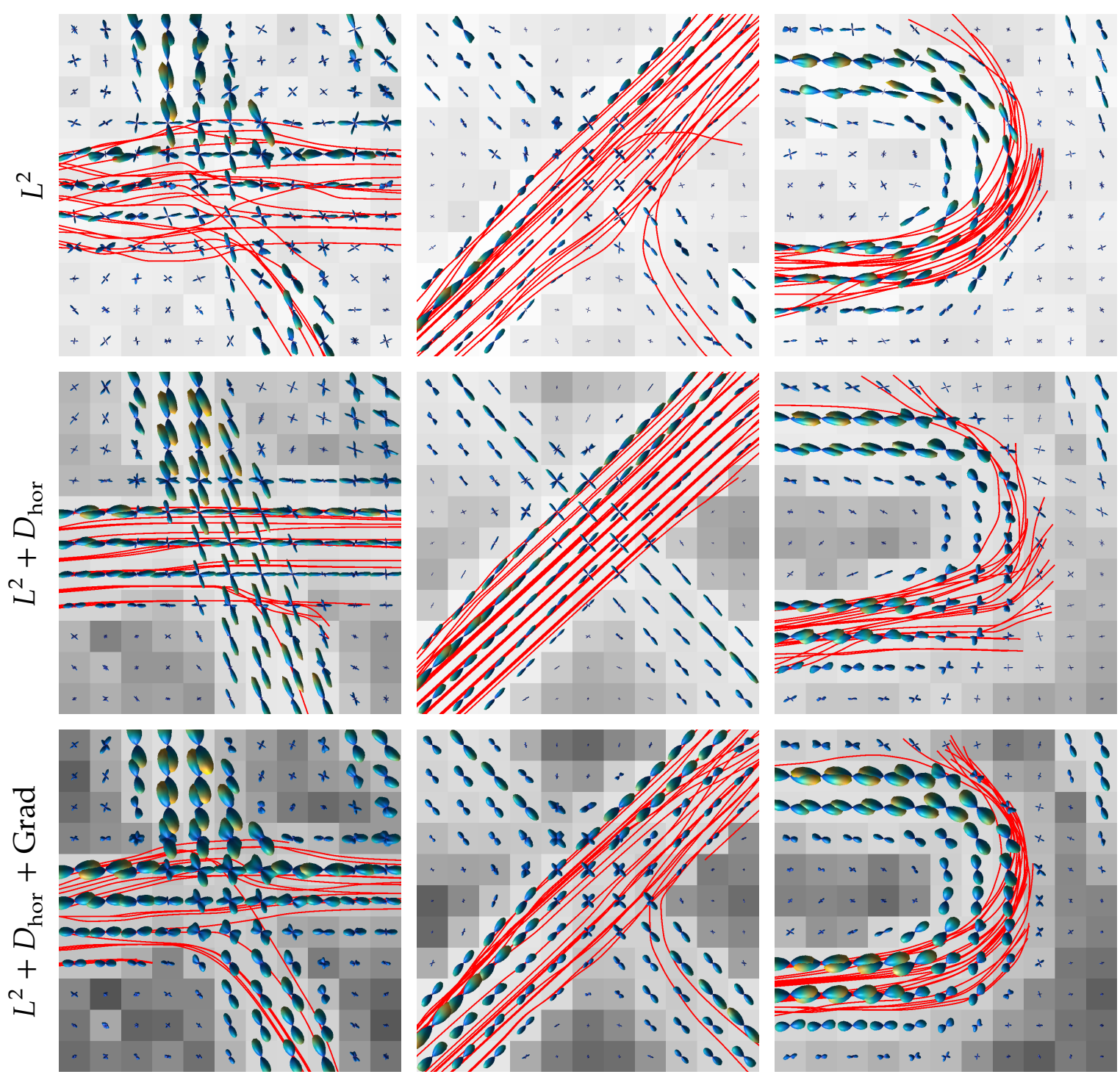

Figure 6.6: Tracking results using Algorithm 6.1 for the spherical deconvolution reconstructions in the SH basis shown in Figure 6.4. The seed regions are: (left) at the left edge, (center) at the top right corner, (right) at the bottom left edge. 

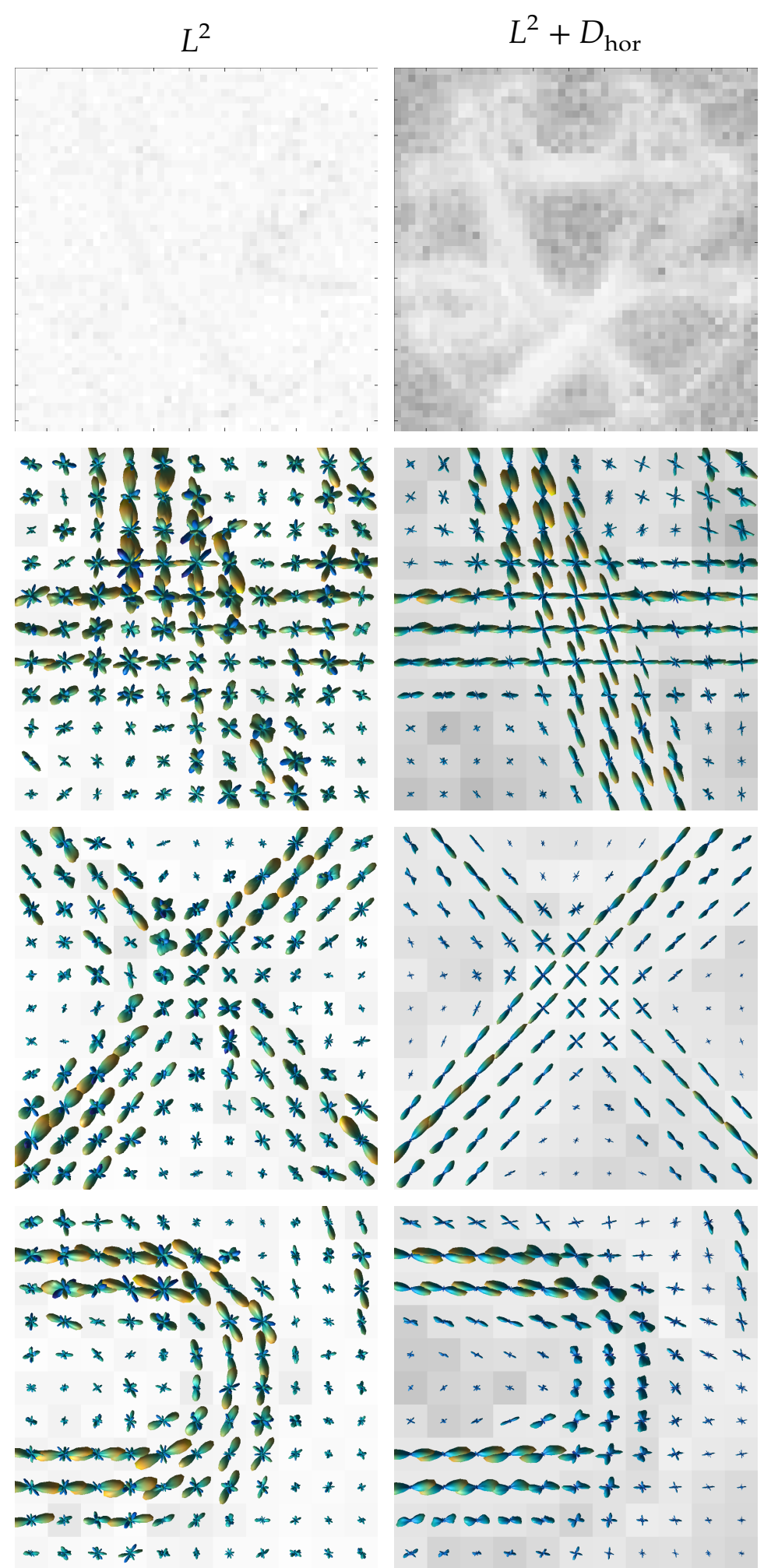

$$
L^{2}+D_{\text {hor }}+\text { Grad }
$$
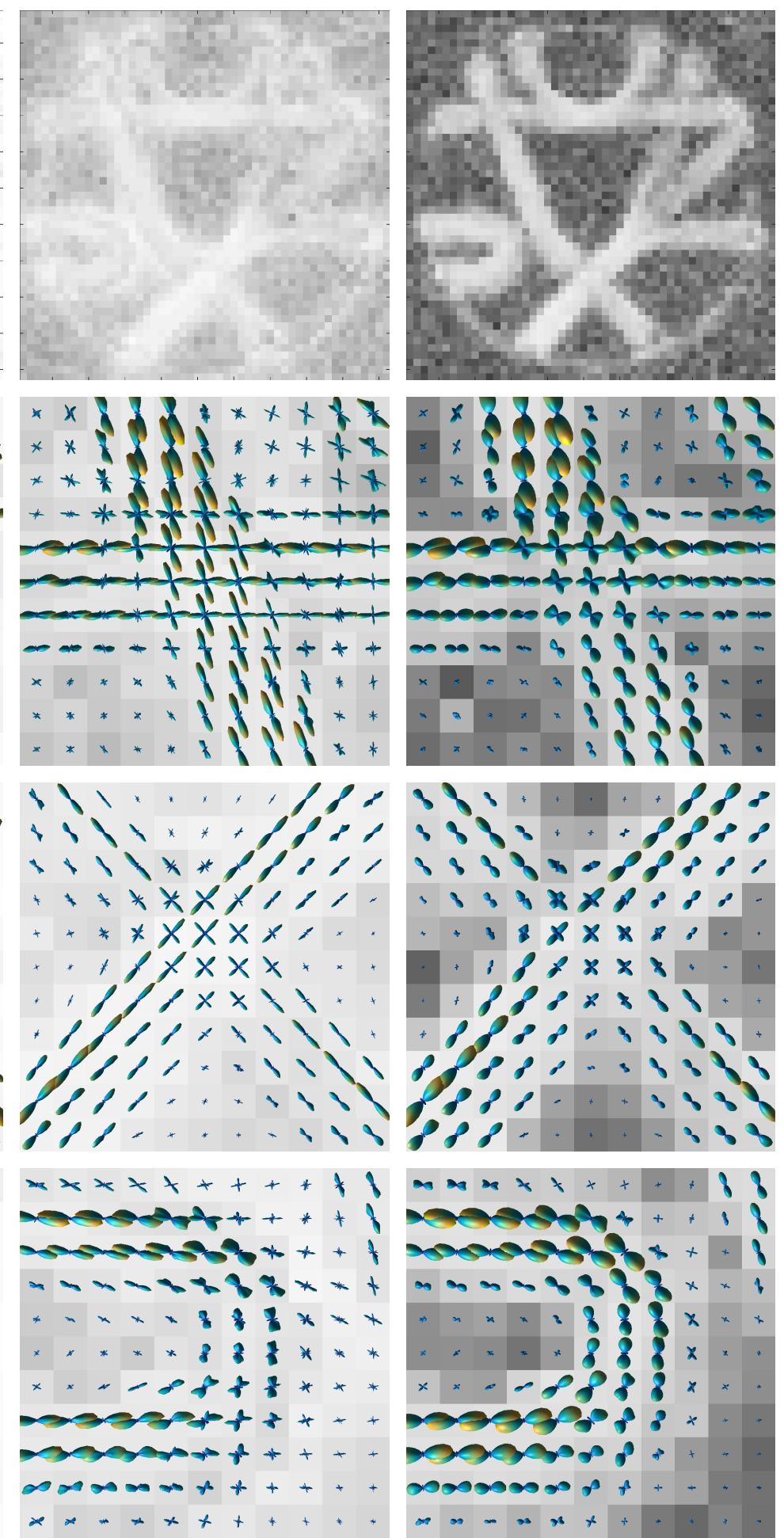

Figure 6.7: Unconstrained spherical deconvolution reconstructions for the highlighted regions of the phantom in Figure 6.2 using the $\mathrm{SH}$ basis, using the same parameters as in Figure 6.4. The top row shows the GFA map. 

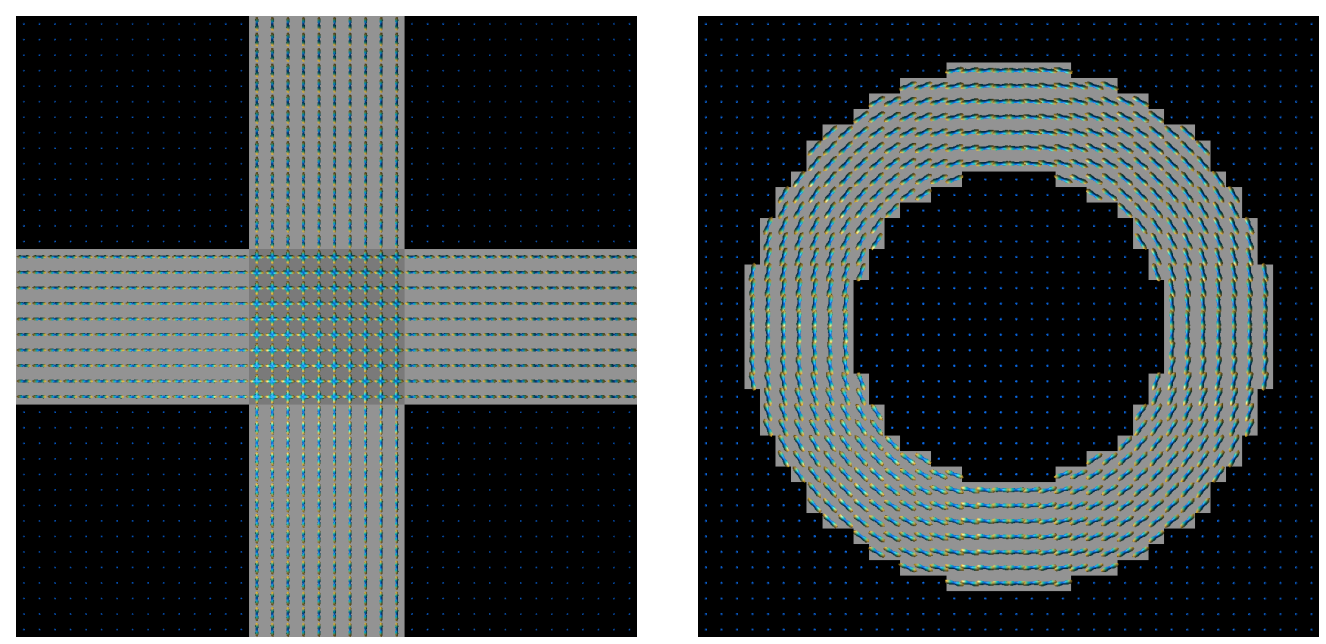

Figure 6.8: Structure of the simulated ODFs for the numerical convergence tests shown in Figures 6.9 and 6.10.

choices of regularization penalty and parameter $\alpha$. The resulting relative reconstruction errors with respect to the $L^{2}$-norm are shown in Figures 6.9 and 6.10.

In all cases, the error shows the balancing between data noise for small $\alpha$ and approximation error for large $\alpha$ that is typical of ill-posed problems, as well as improvement of the reconstructions with decreasing data noise. Also depicted are the optimal relative errors with respect to the noise level for the various penalties, i.e. the best attainable result if there was a method to determine the optimal regularization parameter $\alpha^{*}$ for the given method. In both cases, the spatially regularized methods perform better than pure $L^{2}$ regularization, although the advantage for the curved structure is rather small. A notable effect is that for the straight crossing, the $L^{2}+D_{\text {hor }}$ penalty performs better than the one with additional angular regularization Grad due to over-blurring caused by the latter, while for the curved structure the opposite holds true. This can again be attributed to tangential artifacts as discussed above.

\subsection{Reconstructions of in vivo data and comparison of forward models}

In this section, we test the algorithm on an in-vivo data set for various choices of the forward operator. The data was taken from the Human Connectome Project (HCP) database $^{3}$ and consists of each 90 diffusion weighted images for $b$-values 1000, 2000

\footnotetext{
3See https://ida.Ioni.usc.edu. The HCP project is supported by the National Institute of Dental and Craniofacial Research (NIDCR), the National Institute of Mental Health (NIMH) and the National Institute of Neurological Disorders and Stroke (NINDS). HCP is the result of efforts of co-investigators from the University of Southern California, Martinos Center for Biomedical Imaging at Massachusetts
} 

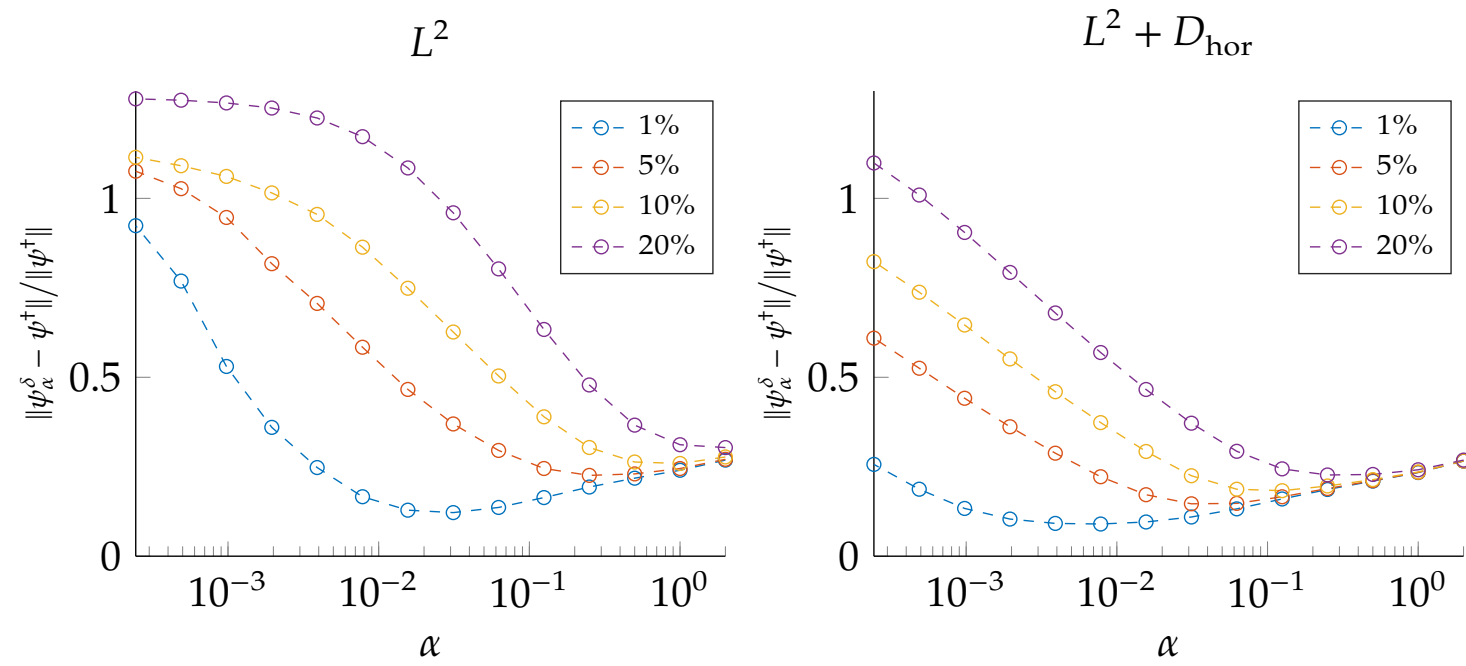

$L^{2}+D_{\text {hor }}+$ Grad
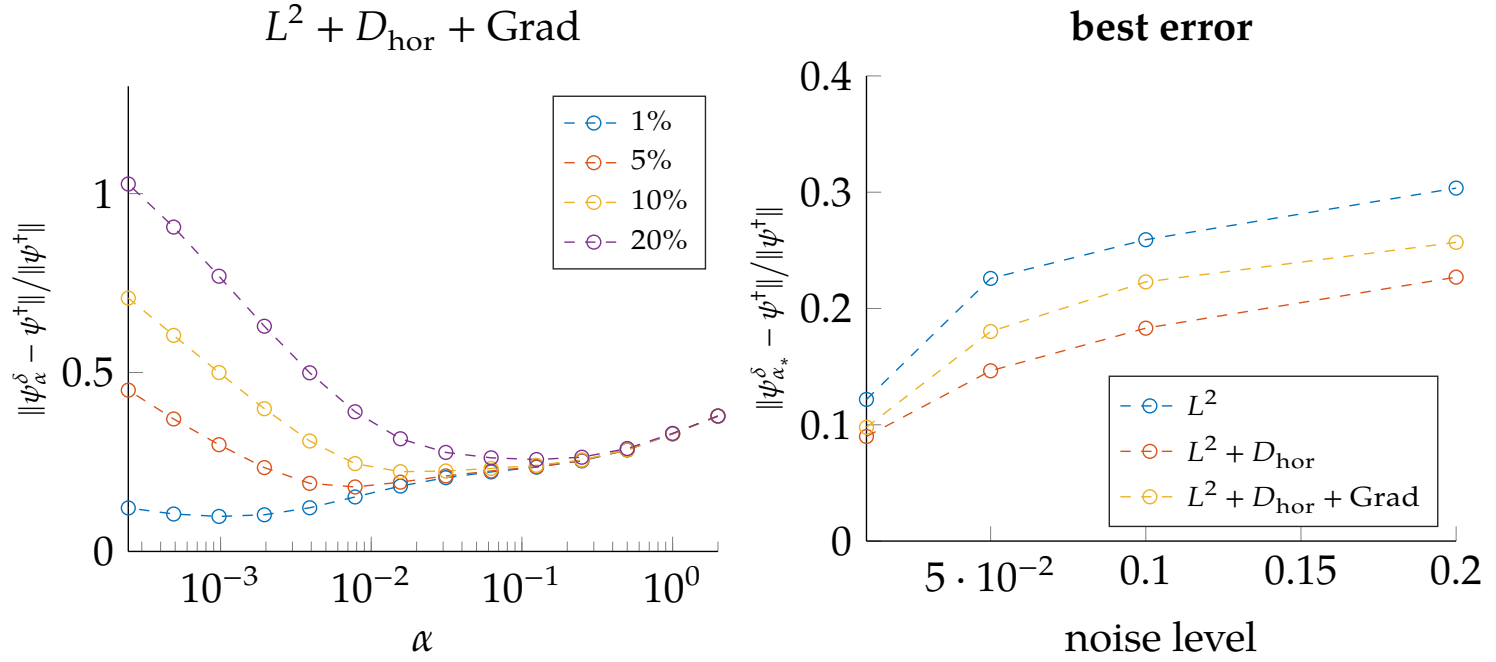

Figure 6.9: Relative errors for reconstructions from simulated data for the cross structure in Figure 6.8 using spherical deconvolution in the $\mathrm{SH}$ basis, for various regularization penalties and levels of Gaussian noise as shown in the legends. The norm on the vertical axis is the $L^{2}$-norm. The bottom right shows shows the errors for the regularization parameter $\alpha_{*}$ that minimizes the respective errors in the other plots, i.e. the best attainable relative error for given noise level and regularization method. 

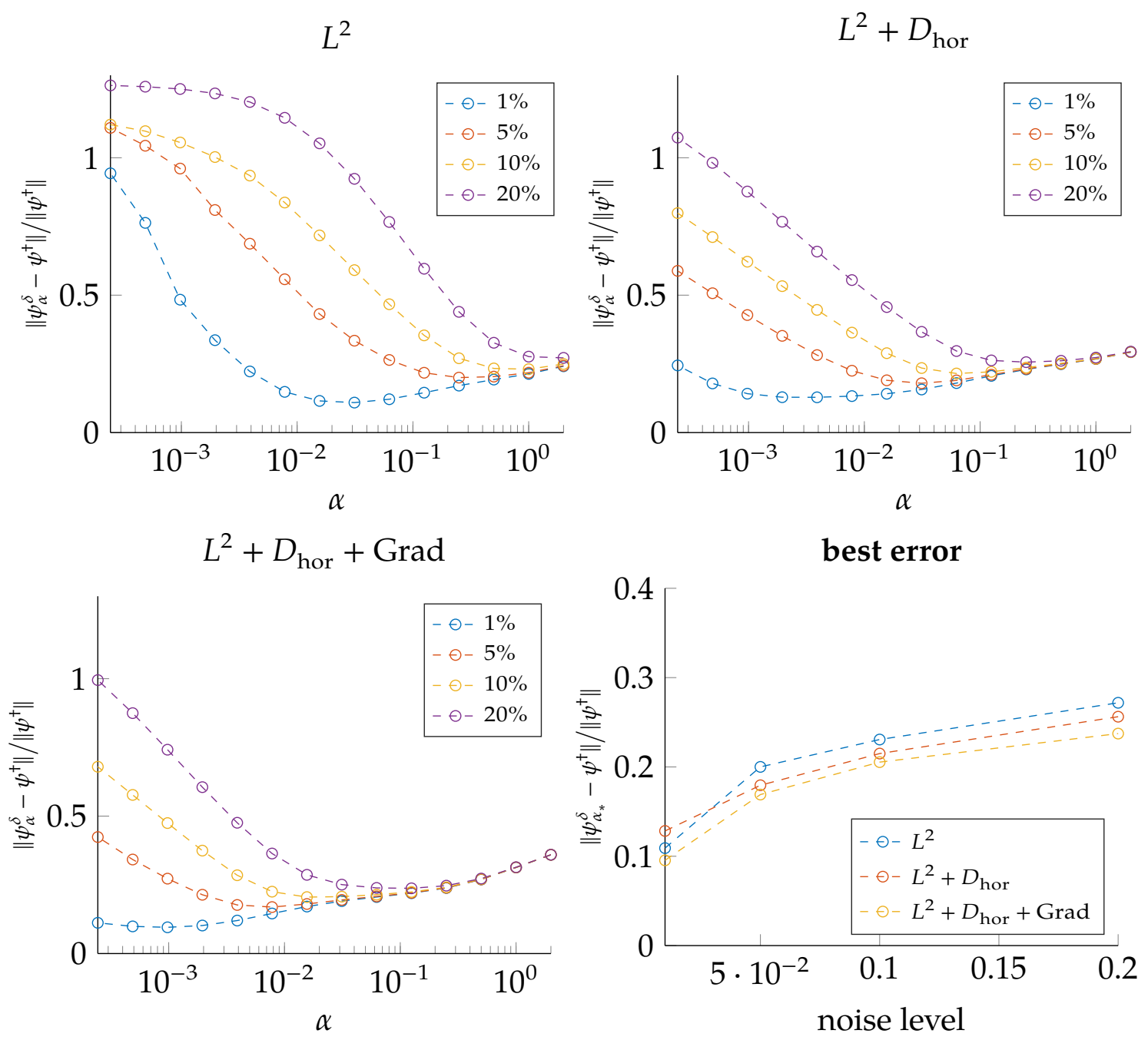

Figure 6.10: Relative errors for reconstructions from simulated data for the circle structure in Figure 6.8 using spherical deconvolution in the $\mathrm{SH}$ basis, for various regularization penalties and levels of Gaussian noise as shown in the legends. The norm on the vertical axis is the $L^{2}$ norm. The bottom right shows shows the errors for the regularization parameter $\alpha_{*}$ that minimizes the respective errors in the other plots, i.e. the best attainable relative error for given noise level and regularization method. 
and $3000 \mathrm{~s} / \mathrm{mm}^{2}$, together with 18 unweighted images, which were averaged to obtain a single base-line image. It should be noted that this is an unusually large data set, which additionally features a good signal-to-noise ratio. The reason is that it is primarily intended for brain research, not for the evaluation of ODF reconstruction and tracking methods. To make the situation more realistic, only a subset of the data will be used. Still, it is interesting to test the performance the method on this data set, in particular to see how well the geometric assumption of locally straight fibers is fulfilled in a realistic situation. Therefore, the spatial regularization parameter was deliberately chosen rather large to highlight some of its strengths and shortcomings.

\subsubsection{Convolution model}

For the convolution model, the kernel was parametrized as a Gaussian with a rotationally symmetric diffusion tensor directed along the $x_{3}$-axis,

$$
D=\lambda_{1} \mathbb{1}+\left(\lambda_{2}-\lambda_{1}\right) e_{3} e_{3}^{T} .
$$

The eigenvalues $\lambda_{1}$ and $\lambda_{2}$ were estimated from the data itself using a straight-forward DTI reconstruction as follows:

- For each voxel $x$ and each $q$ in set of diffusion gradients $\mathcal{Q}$, set

$$
y(x, q):=-\log (S(x, q) / \rho(x)),
$$

where $S$ are the DW weighted images and $\rho$ is the unweighted image. Points where this expression is undefined are discarded.

- Set $\tilde{D}(x):=\operatorname{argmin}_{D} \sum_{q \in \mathcal{Q}}\left|q^{T} D q-y(x, q)\right|^{2}$, where the minimum is taken over all symmetric $3 \times 3$ matrices, and $D(x):=\max (0, \tilde{D}(x))$ in the sense of functional calculus, i.e. truncate negative eigenvalues.

- Discard voxels with $\mathrm{FA}(D(x))<\tau$ for some fixed threshold $\tau$. The remaining voxels are assumed to contain only a single fiber each. For the HCP data set, we used a value of $\tau=0.6$, which was chosen by manual adjustment.

- Let $\left(\tilde{\lambda}_{i}\right)_{i=1}^{3}$ be the averages of the sorted eigenvalues of the $D(x)$ over the remaining voxels $x$. Then $\lambda_{1}=\frac{\tilde{\lambda}_{1}+\tilde{\lambda}_{2}}{2}$ and $\lambda_{2}=\tilde{\lambda}_{3}$.

Better DTI reconstruction techniques are of course available, but this simple and fast approach already lead to good results. The forward operator parametrized by the diffusion tensor above is

$$
T \psi(x, q)=\int_{S^{2}} \exp \left(-b\left(\lambda_{1}-\left(\lambda_{2}-\lambda_{1}\right)(q \cdot u)^{2}\right)\right) \psi(x, u) d u .
$$

General Hospital (MGH), Washington University, and the University of Minnesota. 
Note that the first term in the exponential only leads to an irrelevant normalization constant in this setting.

Reconstructions were performed on a $30 \times 30 \times 35$ voxel subset of the volume around the area shown in Figure 6.11, using only a subset of 40 of the diffusion weighted images and the unscaled version of the forward operator (cf. 2.17), i.e. also ignoring the unweighted images. Only reconstructions using the $\mathrm{SH}$ basis will be shown for simplicity. Due to the smaller number of data points, the $\mathrm{SH}$ order was reduced to 10. Results for $b$-values $1000 \mathrm{~s} / \mathrm{mm}^{2}$ and $2000 \mathrm{~s} / \mathrm{mm}^{2}$ are depicted in Figure 6.12. The $3000 \mathrm{~s} / \mathrm{mm}^{2}$ reconstruction did not differ much from the $2000 \mathrm{~s} / \mathrm{mm}^{2}$ version and will not be shown.

Since the signal usually decays with increasing $b$-value, the SNR of the $1000 \mathrm{~s} / \mathrm{mm}^{2}$ data is the higher, which leads to a better GFA contrast. On the other hand, region (A) shows that the data at smaller $b$-values tends to contain less information on the crossing structures and to appear more blurred. The intuition behind this is that the shorter time between the diffusion sensitizing gradient pulses gives the particles less opportunity to probe the micro-structure of the tissue. Region (A) in also highlight the strength of the $D_{\text {hor }}$ penalty, which significantly improves the coherence of the crossing elongated structures in this area.

Region (B) shows a white matter structure extending into a gray matter area, which may or may not be an artifact due to the general tendency of the penalty to prolong elongated structures. In cases like these, validation based on other sources of knowledge about the local structures is necessary.

Regions (C) and (D) show two opposing effects: region (D) shows spurious crossings introduced into a bending structure similar to what was observed on the phantom data, while in region (C) presumably crossing structures are smoothed out. Note however that the fiber geometry of the region is very complex, with bending, crossing and merging areas very close together. In these situations, it will be very difficult to decide algorithmically which of the solutions is correct based on locally available information, and without knowledge on the specifics of brain anatomy. Therefore, manually guiding the reconstruction may be required, for example by using predetermined, spatially varying regularization parameters.

Another notable feature of the regularized reconstructions is the significantly improved GFA contrast between gray and white matter even without the Laplace-Beltrami penalty. This shows that the spatial penalty is able to distinguish between noise and oriented structures using the fact that the former are not coherent with their surroundings.

\subsubsection{Multi-shell deconvolution}

As discussed in Section 2.2.4, the convolution model can be extended to the multi-shell setting if convolution kernels for the different shells are known. An easy way to obtain 


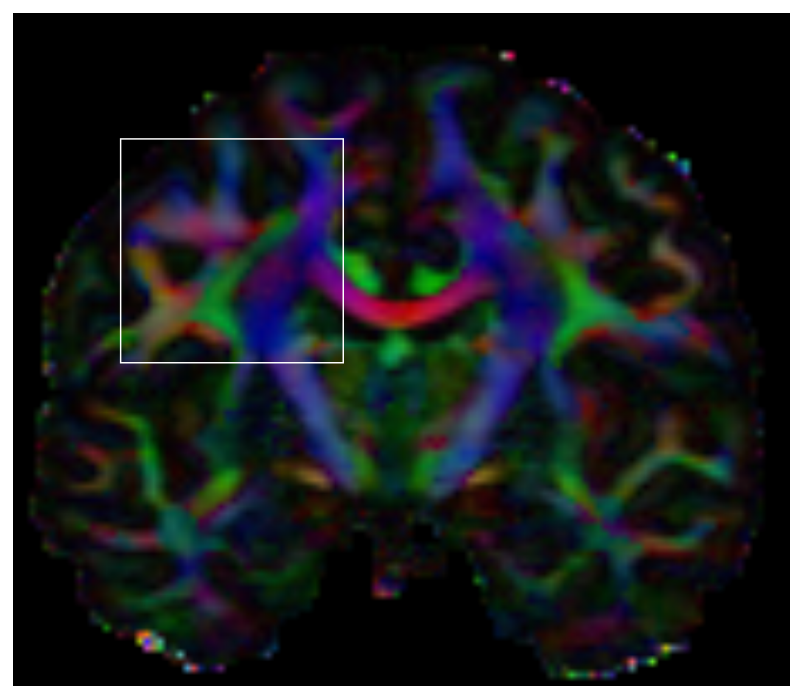

Figure 6.11: Slice of the in-vivo data set from the Human Connectome Project (HCP). The highlights region is the sub-region on which reconstructions were performed (Figures 6.12 to 6.15). The image shows FA color coded by main diffusion direction: (red) left-right, (green) forward-backward, (blue) up-down.

these kernels is to use the DTI-based method described above, either by estimating a single tensor from data on all shells as in (2.19), or by using the method multiple times to estimate one tensor per shell as in (2.21). Choosing each 20 gradient directions for the $b=1000 \mathrm{~s} / \mathrm{mm}^{2}$ and $2000 \mathrm{~s} / \mathrm{mm}^{2}$ shells, we obtain tensor eigenvalues $(0.41,1.44) \times$ $10^{-3} \mathrm{~mm}^{2} / \mathrm{s}$ for the $1000 \mathrm{~s} / \mathrm{mm}^{2}$ shell and $(0.34,1.13) \times 10^{-3} \mathrm{~mm}^{2} / \mathrm{s}$ for the $1000 \mathrm{~s} / \mathrm{mm}^{2}$ shell. Using all data to estimate a single tensor leads to eigenvalues $(0.35,1.19) \times$ $10^{-3} \mathrm{~mm}^{2} / \mathrm{s}$, so the estimates are rather similar, with the $1000 \mathrm{~s} / \mathrm{mm}^{2}$ shell showing a stronger isotropic part as expected. This suggests that at least for these $b$-values, the Gaussian model can be a satisfactory description of the radial behavior of the data. ${ }^{4}$ Moreover, choosing between (2.19) and (2.21) does not play a significant role. Figure 6.13 shows results using the same parameters as for the single-shell case above, for the kernel (2.21). As expected, the reconstruction quality is somewhere in the middle between the 1000 and $2000 \mathrm{~s} / \mathrm{mm}^{2}$ single-shell reconstructions.

\subsubsection{Q-Ball model}

The Q-Ball method (2.16),

$$
\psi=\frac{1}{4 \pi}+\frac{1}{16 \pi^{2}} \Delta_{S^{2}} \mathcal{R} \log \left(-\log \left(\frac{S}{\rho}\right)\right),
$$

\footnotetext{
${ }^{4}$ For larger $b$-values, more complicated effects like diffraction peaks are known to occur [ATBog], so
} the model would break down. 

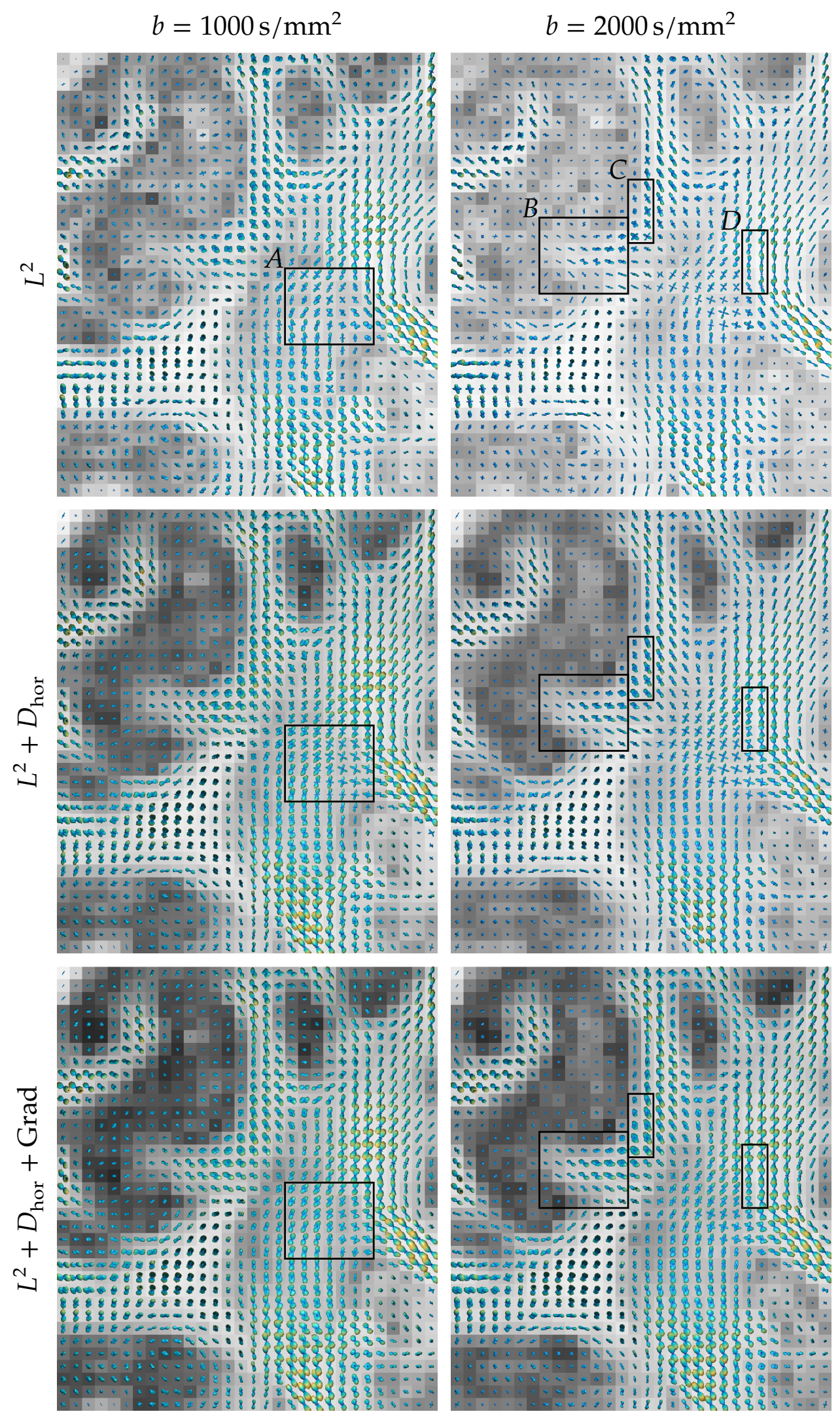

Figure 6.12: Spherical deconvolution reconstructions of the HCP data in the SH basis for the region highlighted in Figure 6.11 from 40 gradient directions and varying $b$-values and penalties. See the text for a discussion of the highlights regions. 

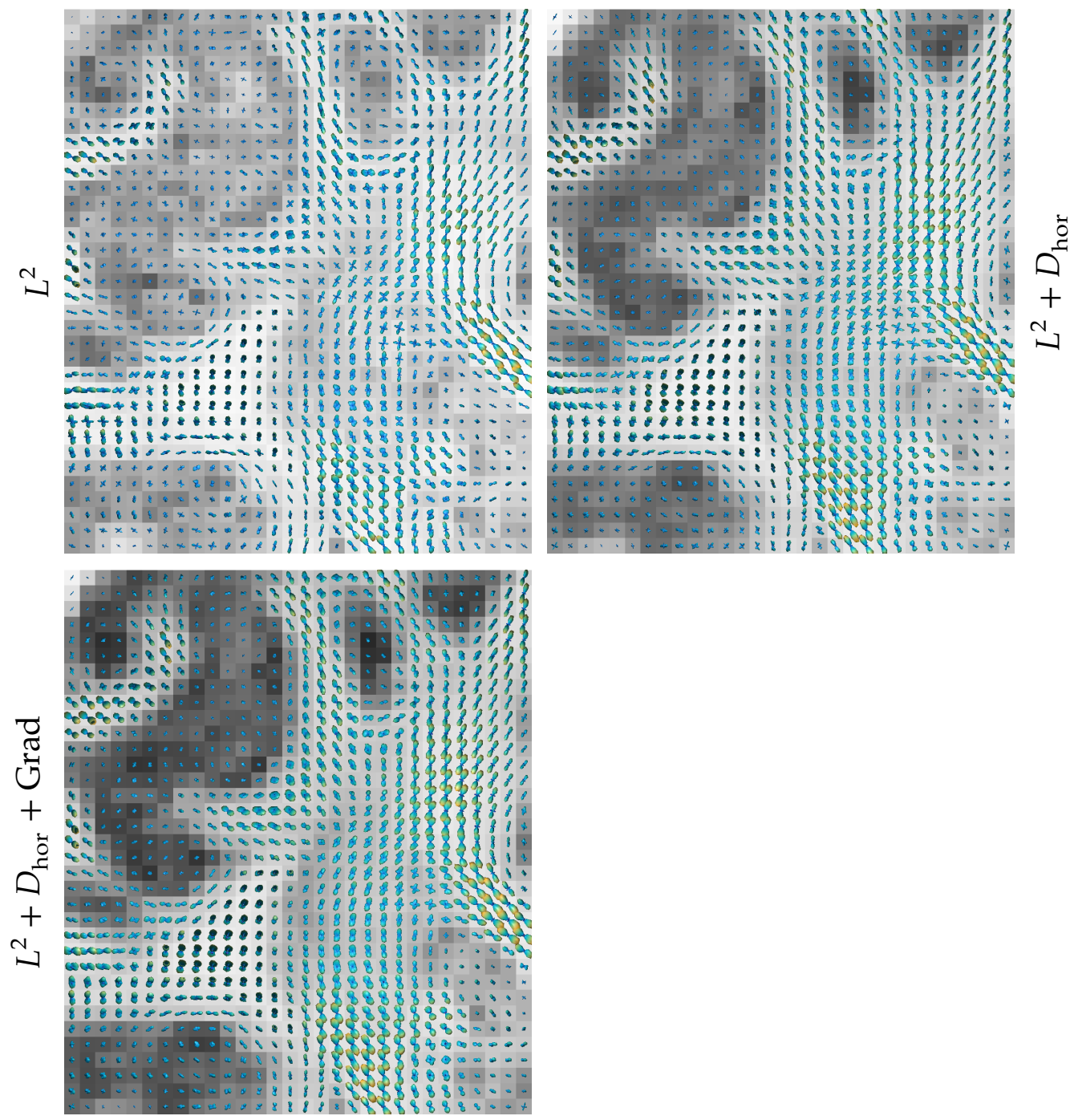

Figure 6.13: Multi-shell spherical deconvolution reconstructions of the HCP data with each 20 gradient directions for $b=1000$ and $2000 \mathrm{~mm}^{2} / \mathrm{s}$ using the $\mathrm{SH}$ basis for the region highlighted in Figure 6.11 with the same parameters as for the single-shell reconstruction Figure 6.12. 
was also tested on the HCP data. This method is most easily implemented in the SH basis, since the linear part $\Delta_{S^{2}} \mathcal{R}$ of the forward operator is rotationally invariant and therefore diagonalized by SHs. As shown in [DAFDo7], the spectral coefficients of the Funk-Radon transform $\mathcal{R}$ are given by the expression

$$
\mathcal{R} Y_{l m}=2 \pi P_{l}(0) Y_{l m}
$$

where $P_{l}$ is the Legendre polynomial of order $l$. Explicitly,

$$
P_{l}(0)= \begin{cases}0 & l \text { odd }, \\ (-1)^{l / 2} \frac{1 \cdot 3 \cdots(l-1)}{2 \cdot 4 \cdots l} & l \text { even. }\end{cases}
$$

Writing this using the $\Gamma$-function, the asymptotic expansion

$$
P_{l}(0)=(-1)^{l / 2} \sqrt{\frac{2 \pi}{l}}+\mathcal{O}\left(\frac{1}{l}\right) \text { as } l \rightarrow \infty, l \text { even }
$$

can be shown. So the operator $\Delta_{S^{2}} \mathcal{R}$ is unbounded, which necessitates a rather low choice for the SH order to avoid instability due to noise. Reconstruction is performed along the lines of [ALS+10] by first computing the double logarithm in the expression above,

$$
y=\log \left(-\log \left(\frac{S}{\rho}\right)\right)
$$

dropping points where this is undefined. The result is then expanded in SHs, optionally including a regularizing penalty for additional stability,

$$
\underset{c}{\operatorname{argmin}}\left(\left\|Y_{c}-y\right\|^{2}+\alpha\|L c\|^{2}\right) \text {. }
$$

Here, $Y$ the SH matrix and $L$ implements $\sqrt{-\Delta_{S^{2}}}$, i.e. a diagonal matrix with entries $\sqrt{l(l+1)}$. Results using SHs of order 6 and all $b=2000 \mathrm{~s} / \mathrm{mm}^{2}$ points are shown in Figure 6.14. Compared to the results shown above, the reconstructions are of noticeably lower quality. Some of the crossings are resolved nicely, but interestingly, the ODFs in single-fiber voxels seem to be much more noisy than above. As before, GFA contrast is improved by Laplace-Beltrami regularization by reducing noise in isotropic areas. The unfavorable result may be due to incorrect modelling assumptions or lack of proper regularization. Therefore, it is interesting to see how the Q-Ball model performs when including the spatial penalty and non-negativity constraint.

To do this, we first formulate (6.1) as an inverse problem,

$$
16 \pi^{2}\left(\Delta_{S^{2}} \mathcal{R}\right)^{-1} \varphi=y-\frac{1}{4 \pi} \int_{S^{2}} y,
$$

where $\varphi=\psi-(4 \pi)^{-1}$. Note that $\Delta_{S^{2}} \mathcal{R}: H_{0}^{3 / 2}\left(S^{2}\right) \rightarrow L_{0}^{2}\left(S^{2}\right)$ is bijective, where $L_{0}^{2}\left(S^{2}\right):=$ $\left\{\varphi \in L^{2}\left(S^{2}\right): \int f=0, f\right.$ even $\}$, and $H_{0}^{3 / 2}\left(S^{2}\right):=H^{3 / 2}\left(S^{2}\right) \cap L_{0}^{2}\left(S^{2}\right)$. So the inverse above makes sense when restricting $\varphi$ to $L_{0}^{2}\left(S^{2}\right)$ in each voxel. 

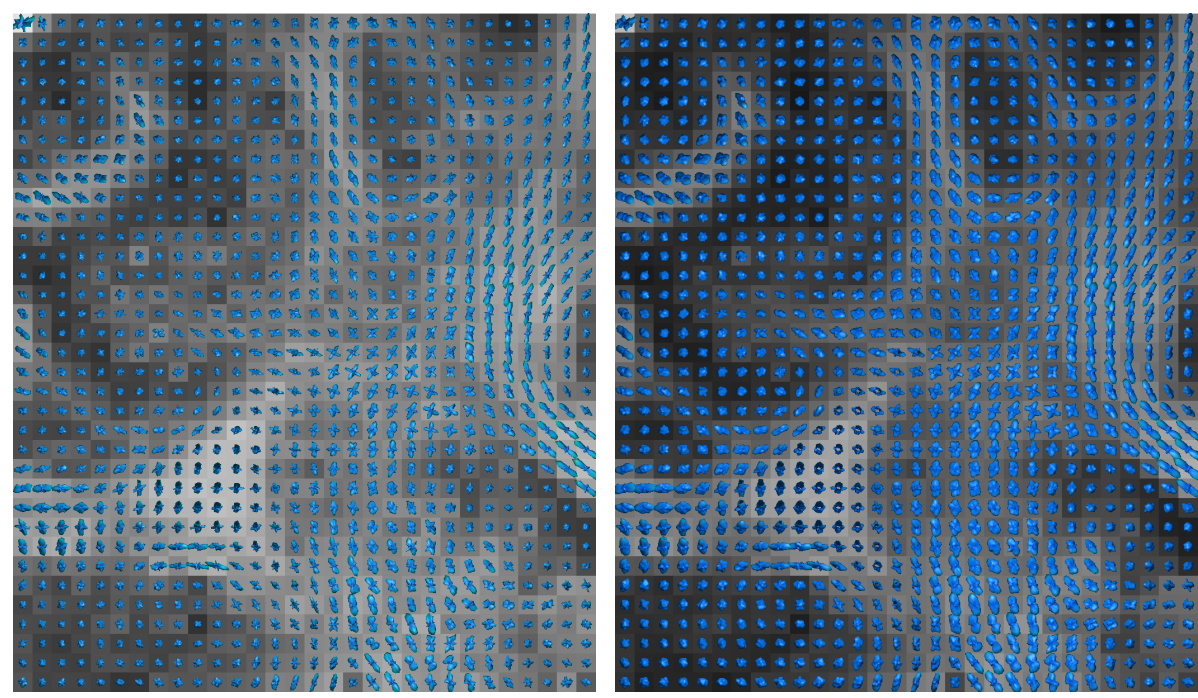

Figure 6.14: Solid-angle Q-Ball reconstructions of HCP data for the region highlighted in Figure 6.11 using the explicit expression (6.1) for 90 gradient directions. (Left): SH coefficients of data determined without regularization in (6.3). (Right): SH coefficients determined with additional Laplace-Beltrami regularization.

Problem (6.4) can actually be viewed as a deconvolution problem, with the convolution kernel given in SHs by the the spectral coefficients of $\left(\Delta_{S^{2}} \mathcal{R}\right)^{-1}$. Therefore, almost the same implementation as above can be used. A few points have to be taken into account:

- There is an additional side condition $\int_{S^{2}} \varphi=0$ to be implemented. This can be done easily by omitting the order 0 SH coefficient of $\varphi$ during reconstruction.

- The constant shift between $\varphi$ and $\psi$ has to be accounted for in the lower bound. In the Moreau-Yosida regularized method, this can be done by replacing (5.22) with

$$
\underset{H x \geq w}{\operatorname{argmin}}\left(\|T x-y\|^{2}+c\|w-a\|^{2}\right),
$$

where $a$ is the lower bound, and otherwise following the same steps as above.

- The double logarithm (6.2) changes the noise statistics, so that a quadratic data penalty is likely not an optimal choice. To be precise, even for the deconvolution model, the noise is not Gaussian due to using magnitude data in equation (2.11) (the resulting noise usually follows is a Rice distribution, cf. [GP95], but is even more complicated for parallel imaging). On the other hand, inverting the double logarithm would lead to a non-linear inverse problem involving a double exponential, which is likely to lead to poor convergence even for Newton-type methods. For the sake of simplicity, we will still use a quadratic data penalty, but this is a problem that should be handled more thoroughly. 
Reconstructions are shown in Figure 6.15. The $L^{2}$ and $L^{2}+D_{\text {hor }}+$ Grad are comparable to the unregularized and regularized versions above, respectively, while the purely spatially regularized reconstruction achieves good coherence without the over-blurring introduced by the Laplace-Beltrami operator. In all cases, a notable effect of the model is that the requirement $\int_{S^{2}} \psi=1$ leads to comparably large ODFs in regions with no or only isotropic diffusion, which makes it difficult to locally distinguish between these and for example the large oriented structure in the lower part of the images. This is of course inherent in the choice of the solid-angle ODF $\psi=\psi_{2}$ in (2.16). Altogether, the Q-Ball model appears to be an inferior choice compared to the Gaussian convolution model, at least for this data set. 

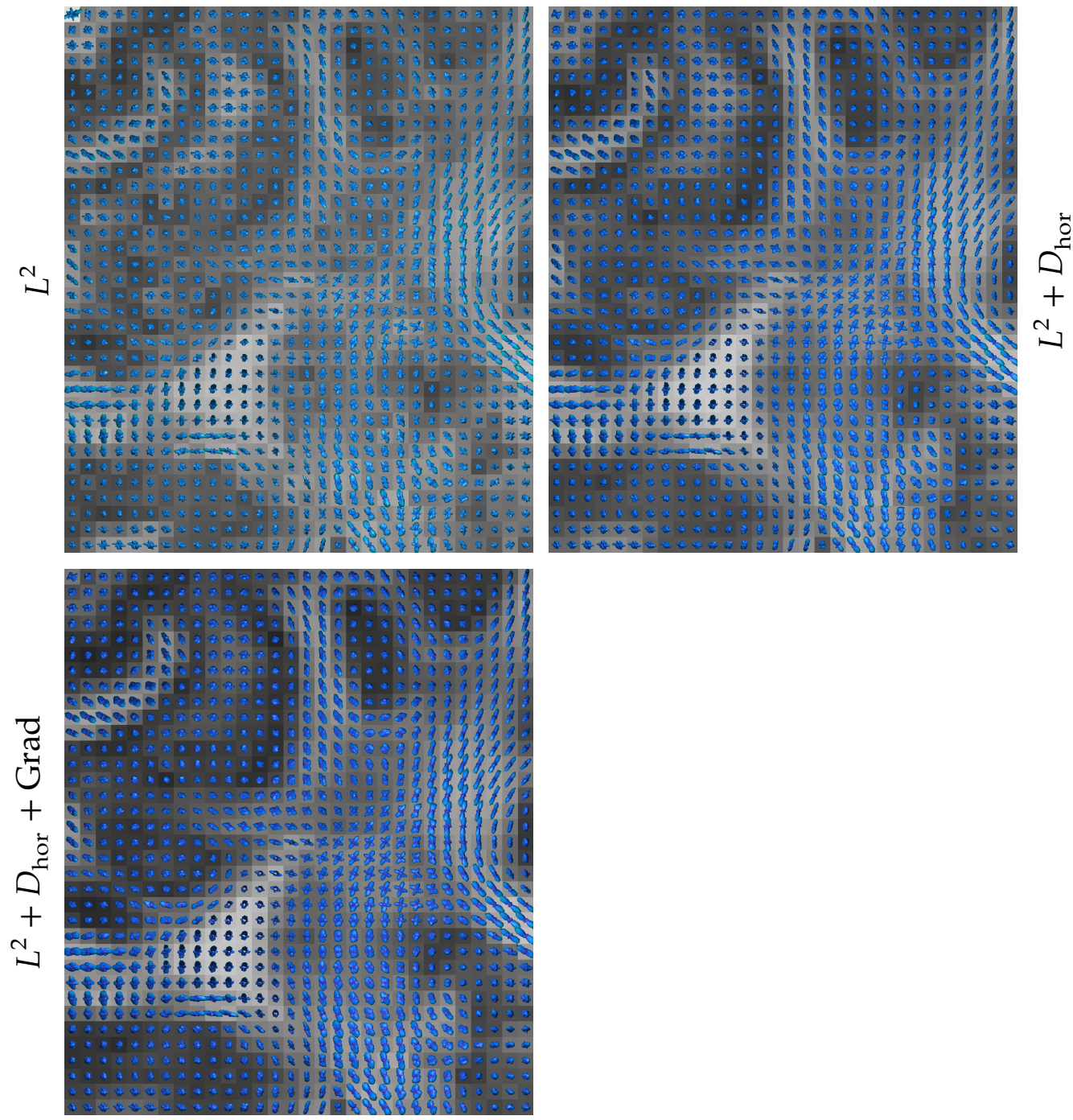

Figure 6.15: Solid-angle Q-Ball reconstructions of HCP data for the region highlighted in Figure 6.11 for 90 gradient directions, obtained by writing the Q-Ball operator as regularized spherical deconvolution in the $\mathrm{SH}$ basis as described in the text, and using various regularization penalties. 



\section{Summary}

The aim of this thesis was to investigate the possibilities for enhancing the spatial coherence of ODF reconstructions from coarsely sampled, low SNR data, where in contrast to pre- or post-processing approaches, reconstruction and smoothing were carried out in a single step. Motivated by existing results on diffusion filters on $\mathrm{SE}(3) / \mathrm{SO}(2) \simeq \mathbb{R}^{3} \times S^{2}$, we introduced a penalty for smoothing ODFs that compares ODFs in neighboring voxels locally in the orientational part, comparing each orientational component only along the corresponding spatial direction.

The Tikhonov-type regularization method using this penalty is naturally defined on a non-standard Sobolev space, which was thoroughly analyzed, in particular proving a theorem on compact embedding into $L^{2}$. As a variation of a known result on generalized Tikhonov regularization with approximate operator, we proved convergence of reconstructions from discrete data for compact forward operators, if the discretization is performed appropriately and the exact solution fulfills a variational smoothness assumption. Combining this with the embedding theorem, we were able to obtain convergence of our regularization strategy for discrete, noisy data and linear forward operators.

We described efficient implementation of our regularization strategy as a projection method and analyzed the approximation properties of the corresponding discrete spaces, showing that the projections in the domain fulfill the properties required by the convergence result. For the codomain, our implementation deviates from the precise requirements due to details of the physical measurement, but we expect the difference not to play a major role in practice - an expectation that is also confirmed by our numerical experiments.

We described two possible solvers for the resulting constrained optimization problem. The first one - the primal-dual active set method - is known have good convergence properties, and is further accelerated by the duality-gap based stopping rule. We also used an ad-hoc minimization algorithm that is known from constrained voxel-wise reconstructions of DW data using Spherical Harmonics, and were able to justify the algorithm by identifying it as a form of Moreau-Yosida regularization of the non-negativity constraint.

In the final chapter, we numerically verified convergence of the discretizing projections with respect to refinement of the discretization. This part also illustrated the need to enforce some angular smoothness of the reconstructions in order to obtain convergence. 
Further, we tested convergence of the regularization method with respect to the noise level on simulated data, and, in a more qualitative fashion, analyzed the performance of the method on phantom and in-vivo data, including some simple fiber tracking results for the phantom. This showed significant improvements in spatial coherence and noise reduction compared to voxel-wise approaches, but also highlighted the main shortcoming of the method, i.e. the introduction of artifacts in regions containing fibers with high curvature. We showed that these can be reduced by mild angular regularization, but since this also limits the achievable resolution in crossing areas, it is necessary to balance the penalties appropriately. Until now, this has to be done manually. Due to complex geometry of nerve fibers in the brain, it is likely that some level of manual intervention is unavoidable.

As a perspective, there are several possible extensions to our method to address this problem. One way may be to make use of the convergence with respect to spatial discretization to reconstruct the ODFs on a grid finer than the one on which the data is given, thereby effectively reducing the curvature of non-straight structures and making them conform better to the smoothness assumptions. Another option would be to adaptively choose the regularization parameters and in particular the relative balance between spatial and angular derivatives, depending on the local structure; unfortunately, it is not entirely clear how to approach this, since SE(3)-based orientation estimation turned out not give reliable results. A third option would be to use non-linear versions of the penalty, as in (4.10), to incorporate ideas from non-linear diffusion filters. All of these approaches will, however, lead to a considerable increase in computational complexity. 


\section{Bibliography}

[ALS+10] I. Aganj, C. Lenglet, G. Sapiro, et al. "Reconstruction of the orientation distribution function in single- and multiple-shell q-ball imaging within constant solid angle". In: Magn. Reson. Med. 64.2 (2010), pp. 554-566. Dor: $10.1002 / \mathrm{mrm} .22365$.

[ATBog] H.-E. Assemlal, D. Tschumperlé, and L. Brun. “Efficient and robust computation of PDF features from diffusion MR signal". In: Med. Image Anal. 13.5 (2009), pp.715-729. DoI: 10.1016/j.media.2009.06.004.

[Bako2] A. Baker. Matrix groups: An introduction to Lie theory. Springer, 2002.

[BBJ+07] T. E. J. Behrens, H. J. Berg, S. Jbabdi, et al. “Probabilistic diffusion tractography with multiple fibre orientations: What can we gain?" In: NeuroImage 34.1 (2007), pp. 144-155. Dor: 10.1016/j. neuroimage.2006.09.018.

[BIK99] M. Bergounioux, K. Ito, and K. Kunisch. "Primal-Dual Strategy for Constrained Optimal Control Problems". In: SIAM J. Control Optim. 37.4 (1999), pp. 1176-1194. DOI: 10.1137/S0363012997328609.

[Blo46] F. Bloch. "Nuclear Induction”. In: Phys. Rev. 70.7-8 (1946), pp. 460-474. DOI: $10.1103 /$ physrev . 70.460.

[BSo2] S. C. Brenner and L. R. Scott. The mathematical theory of finite element methods. 2nd ed. Texts in Applied Mathematics 15. Springer, 2002.

[BTV+12] S. Becker, K. Tabelow., H. U. Voss, et al. "Position-orientation adaptive smoothing of diffusion weighted magnetic resonance data (POAS)". In: Med. Imag. Anal. 16.6 (2012), pp. 1142-1155. Dor: $10.1016 /$ j.media. 2012. 05.007.

[Como9] P. L. Combettes. "Iterative construction of the resolvent of a sum of maximal monotone operators". In: J. Convex. Anal. 16 (2009), pp. 727-728.

[DAFDo7] M. Descoteaux, E. Angelino, S. Fitzgibbons, and R. Deriche. "Regularized, fast and robust analytical Q-Ball imaging". In: Magn. Reson. Med. 58.3 (2007), pp. 497-510. DOI: 10.1002/mrm. 21277.

[DCGD11] R. Duits, E. Creusen, A. Ghosh, and T. Dela Haije. Diffusion, convection and erosion on $\mathrm{SE}(3) /(\{0\} \times \mathrm{SO}(2))$ and their application to the enhancement of crossing fibers. 2011. arXiv: 1103.0656v5 [math. AP]. 
[DDL+o1] M. Descoteaux, R. Deriche, D. Le Bihan, et al. "Multiple q-shell diffusion propagator imaging". In: Med. Image Anal. 15.4 (2001), pp. 603-621. Dor: 10.1016/j.media.2010.07.001.

[DF11] R. Duits and E. Franken. "Left-invariant diffusions on the space of positions and orientations and their application to crossing-preserving smoothing of HARDI images". In: Int. J. Comput. Vis 92.3 (2011), pp. 231-264. DOI: 10.1007/s11263-010-0332-z.

[Duio5] R. Duits. "Perceptual organization in image analysis". PhD thesis. Eindhoven University of Technology, Department of Biomedical Engineering, 2005 .

[Dyk83] R. L. Dykstra. "An algorithm for restricted least squares regression". In: J. Am. Stat. Assoc. 78.384 (1983), pp. 837-842. DoI: 10.1080/01621459.1983. 10477029.

[EHN96] H. Engl, M. Hanke, and A. Neubauer. Regularization of inverse problems. Mathematics and Its Applications. Kluwer Academic Press, 1996.

[ET76] I. Ekeland and R. Temam. Convex analysis and variational problems. NorthHolland, 1976.

[FDG+11] P. Fillard, A. Descoteaux M. Goh, S. Gouttard, et al. “Quantitative evaluation of 10 tractography algorithms on a realistic diffusion MR phantom". In: Neuroimage 56.1 (2011), pp. 220-234. Dor: 10.1016/j . neuroimage . 2011. 01.032.

[FH11] J. Flemming and B. Hofmann. "Convergence rates in constrained Tikhonov regularization: equivalence of projected source conditions and variational inequalities". In: Inverse Probl. 27.8 (2011), p. 085001. DoI: 10.1088/02665611/27/8/085001.

[Fle13] J. Flemming. "Variational smoothness assumptions in convergence rate theory - an overview". In: J. Inverse Ill-Posed Probl. 21.3 (2013), pp. 395-409. DOI: $10.1515 / \mathrm{jip}-2013-0001$.

[Frao8] E. Franken. "Enhancement of crossing elongated structures in images". $\mathrm{PhD}$ thesis. Eindhoven University of Technology, 2008.

[GLTVog] A. Goh, C. Lenglet, P. M. Thompson, and R. Vidal. “Estimating orientation distribution functions with probability density constraints and spatial regularity". In: Medical Image Computing and Computer-Assisted Intervention 2009. Ed. by G.-Z. Yang, D. Hawkes, D. Rueckert, et al. Vol. 5761. Lecture Notes in Computer Science. Springer Berlin Heidelberg, 2009, pp. 877-885. DOI: $10.1007 / 978-3-642-04268-3 \_108$.

[GP95] H. Gudbjartsson and S. Patz. "The Rician distribution of noisy MRI data". In: Magn. Reson. Med. 34.6 (1995), pp. 910-914. Dor: 10.1002/mrm. 1910340618.

[Hah5o] E. L. Hahn. "Nuclear induction due to free Larmor precession". In: Phys. Rev. 77.2 (1950), pp. 297-298. Dor: 10.1103/physrev.77.297.2. 
[HIKo3] M. Hintermüller, K. Ito, and K. Kunisch. “The primal-dual active set strategy as a semismooth Newton method". In: SIAM J. Optim. 13.3 (2003), pp. 865-888. DOI: 10.1137/S1052623401383558.

[HKPSo7] B. Hofmann, B. Kaltenbacher, C. Pöschl, and O. Scherzer. “A convergence rates result for Tikhonov regularization in Banach spaces with non-smooth operators". In: Inverse Probl. 23.3 (2007), pp. 987-1010. Dor: 10.1088/02665611/23/3/009.

[Hör67] L. Hörmander. "Hypoelliptic second order differential equations". In: Acta Mathematica 119.1 (1967), pp. 147-171. DOI: $10.1007 /$ bf 02392081.

[HR14] T. Hohage and C. Rügge. A coherence enhancing penalty for Diffusion MRI: regularizing property and discrete approximation. 2014. arXiv: 1412.1439 [math.AP]. Submitted.

[HW13] T. Hohage and F. Werner. "Iteratively regularized Newton-type methods for general data misfit functionals and applications to Poisson data". In: Numer. Math. 123.4 (2013), pp. 745-779. Dor: 10.1007/s00211-012-0499-z.

[HY10] B. Hofmann and M. Yamamoto. “On the interplay of source conditions and variational inequalities for nonlinear ill-posed problems". In: Appl. Anal. 89.11 (2010), pp. 1705-1727. DOI: 10.1080/00036810903208148.

[IKoo] K. Ito and K. Kunisch. "Augmented Lagrangian methods for nonsmooth, convex optimization in Hilbert Spaces". In: Nonlinear Anal. 41 (2000), pp. 591-616. Dor: 10.1016/S0362-546X (98)00299-5.

[JAo3] K. Jansons and D. Alexander. "Persistent angular structure: new insights from diffusion magnetic resonance imaging data". In: Inverse Probl. 19.5 (2003), pp. 1031-1046. Dor: 10.1088/0266-5611/19/5/303.

[LF12] S. Lu and J. Flemming. "Convergence rate analysis of Tikhonov regularization for nonlinear ill-posed problems with noisy operators". In: Inverse Probl. 28.10 (2012), p. 104003. DOI: 10.1088/0266-5611/28/10/104003.

[Nédo1] J. Nédélec. Acoustic and electromagnetic equations. Springer, 2001.

[Neu88] A. Neubauer. "Tikhonov-regularization of ill-posed linear operator equations on closed convex sets". In: J. Approx. Theory 53.3 (1988), pp. 304-320. DOI: 10 . 1016/0021-9045(88)90025-1.

[NHo5] F. Nier and B. Helffer. Hypoelliptic estimates and spectral theory for FokkerPlanck operators and Witten Laplacians. Lecture Notes in Mathematics. Springer, 2005. DOI: $10.1007 / \mathrm{b} 104762$.

[Økso3] B. K. Øksendal. Stochastic differential equations: An introduction with applications. Springer, 2003.

[ÖSV+o6] E. Özarslan, T. M. Shepherd, B. C. Vemuri, et al. "Resolution of complex tissue microarchitecture using the diffusion orientation transform (DOT)". In: NeuroImage 31.3 (2006), pp. 1086-1103. Dor: $10.1016 / \mathrm{j}$. neuroimage . 2006.01 .024$. 
[PV9o] R. Plato and G. Vainikko. “On the regularization of projection methods for solving ill-posed problems". In: Numer. Math. 57.1 (1990), pp. 63-79. DOI: $10.1007 / \mathrm{bf} 01386397$.

[RK11] M. Reisert and V. G. Kiselev. "Fiber continuity: an anisotropic prior for ODF estimation". In: IEEE Trans. Med. Imaging 30.6 (2011), pp. 1274-1283. DOI: $10.1109 /$ TMI.2011.2112769.

[RMA+11] M. Reisert, I. Mader, C. Anastasopoulos, et al. “Global fiber reconstruction becomes practical". In: NeuroImage 54.2 (2011), pp. 955-962. Dor: 10.1016/ j.neuroimage.2010.09.016.

[RR93] J. B. Ra and C. Y. Rim. "Fast imaging using subencoding data sets from multiple detectors". In: Magn. Reson. Med. 30.1 (1993), pp. 142-145. Dor: $10.1002 / \mathrm{mrm} .1910300123$.

[Sch15] H. Schomburg. "Semi-local tractography strategies using neighborhood information". 2015. In preparation.

[SGG+09] O. Scherzer, M Grasmair, H Grossauer, et al. Variational methods in imaging. Applied Mathematical Sciences. Springer, 2009. Dor: 10 .1007/978-0-38769277-7.

[ST65] E. O. Stejskal and J. E. Tanner. "Spin diffusion measurements: Spin echoes in the presence of a time-dependent field gradient". In: J. Chem. Phys. 42.1 (1965), pp. 288-292. DOI: 10.1063/1.1695690.

[Ste65] E. O. Stejskal. "Use of spin echoes in a pulsed magnetic-field gradient to study anisotropic, restricted diffusion and flow". In: J. Chem. Phys. 43.10 (1965), pp. 3597-3603. Dor: 10.1063/1.1696526.

[Tay96] M. Taylor. Partial differential equations: Basic theory. Springer, 1996.

[TCCo7] J. Tournier, F. Calamante, and A. Connelly. "Robust determination of the fibre orientation distribution in diffusion MRI: Non-negativity constrained super-resolved spherical deconvolution". In: NeuroImage 35.4 (2007), pp. 1459-1472. Dor: 10.1016/j . neuroimage.2007.02.016.

[TCC12] J. Tournier, F. Calamante, and A. Connelly. "MRtrix: Diffusion tractography in crossing fiber regions". In: Int. J. Imag. Syst. Tech. 22.1 (2012), pp. 53-66. Dor: $10.1002 / \mathrm{ima} .22005$.

[TCGCo4] J. Tournier, F. Calamante, D. G. Gadian, and A. Connelly. “Direct estimation of the fiber orientation density function from diffusion-weighted MRI data using spherical deconvolution". In: NeuroImage 23.3 (2004), pp. 1176-1185. DoI: $10.1016 / j$. neuroimage. 2004.07.037.

[Tor56] H. C. Torrey. "Bloch equations with diffusion terms". In: Phys. Rev. 104.3 (1956), pp. 563-565. DOI: 10.1103/physrev.104.563.

[Tuco4] D. Tuch. "Q-ball imaging”. In: Magn. Reson. Med. 52.6 (2004), pp. 13581372. DOI: $10.1002 / \mathrm{mrm} .20279$. 
[UHBFo8] M. Uecker, T. Hohage, K. Block, and J. Frahm. “Image reconstruction by regularized nonlinear inversion - Joint estimation of coil sensitivities and image content". In: Magn. Reson. Med. 60.3 (2008), pp. 674-682. Dor: $10.1002 / \mathrm{mrm} .21691$.

[XZC+99] R. Xue, P. C. M. van Zijl, B. J. Crain, et al. "In vivo three-dimensional reconstruction of rat brain axonal projections by diffusion tensor imaging". In: Magn. Reson. Med. 42.6 (1999), pp. 1123-1127. Dor: 10.1002/ (SICI) 15222594(199912) 42:6<1123: :AID-MRM17>3. 0. CO ;2-H. 



\section{Danksagung}

Zum Schluss möchte ich mich bei allen Menschen bedanken, die dieses Projekt ermöglicht und mich unterstützt haben. Zunächst bei meinem Betreuer Professor Thorsten Hohage für die Aufnahme als Promotions-Student, für Diskussionen, Ratschläge und Motivation, und bei meinem Zweit-Betreuer Professor Jens Frahm und seiner Arbeitsgruppe, darunter insbesondere Sabine Hofer, für Zusammenarbeit, Erklärungen und Unterstützung beim nicht-mathematischen Teil des Projekts.

Danke an all die Mitglieder der Arbeitsgruppe Inverse Probleme, die ich im Laufe der Zeit kennenlernen durfte, allen voran meinem ehemaligen Büronachbarn Frank für seine Kombination aus Hilfsbereitschaft und umfangreichem mathematischen Wissen, Robert für ausführliche Gespräche über Mathematisches und Nicht-Mathematisches, und Helen für die enge Zusammenarbeit und für den Tracking-Algorithmus.

Mein Dank gilt auch dem Graduiertenkolleg 1023 „Identifikation in mathematischen Modellen" für finanzielle Unterstützung durch das Stipendium und für die Möglichkeit zu hilfreichen Austausch mit den anderen KollegiatInnen.

Auch ausser-universitär haben viele Menschen zum Gelingen des Projekts beigetragen. Besonderer Dank geht an meine Eltern für Unterstützung und Rückhalt über all die Jahre, und an Melanie, ohne die vor allem die Endphase des Projekts schwer zu bewältigen gewesen wäre. Am Schluss danke ich allen, die die Zeit während der Promotion durch ihre Gesellschaft bereichert haben: Aleks, Boris, Fabian, Felix, Marie, Marlène, Moritz, Petra, Thomas, und, zu guter Letzt, der K1.

Zum Teufel mit Flanders. 